\title{
Numerical Evaluation of State of the Art Horn Designs for Rotary Ultrasonic Vibration Assisted Machining of Nomex Honeycomb Composite
}

Khurram Hameed Mughal ( $\nabla$ khurram.hameed@me.uol.edu.pk )

The University of Lahore, Lahore, Pakistan

Muhammad Asif Mahmood Qureshi

University of Engineering and Technology

Asif Ali Qaiser

University of Engineering and Technology

Fazal Ahmad Khalid

University of Engineering and Technology

\section{Research Article}

Keywords: ultrasonic horn design, finite element analysis, hybrid horns, harmonic analysis, modal analysis, magnification, stresses

Posted Date: February 18th, 2021

DOl: https://doi.org/10.21203/rs.3.rs-207300/v1

License: (c) (i) This work is licensed under a Creative Commons Attribution 4.0 International License. Read Full License 


\title{
Numerical Evaluation of State of the Art Horn Designs for Ultrasonic Vibration Assisted Machining of Nomex Honeycomb Composite
}

\author{
Khurram Hameed Mughal*1,2, Muhammad Asif Mahmood Qureshi ${ }^{1}$, Asif Ali Qaiser ${ }^{1}$, Fazal Ahmad \\ Khalid $^{1}$ \\ ${ }^{1}$ University of Engineering and Technology, Lahore 54890, Pakistan \\ ${ }^{2}$ The University of Lahore, Lahore 54000, Pakistan \\ *Corresponding author Email: khurram.hameed@me.uol.edu.pk
}

\begin{abstract}
Ultrasonic horn plays vital role in achieving vibration amplitude suitable for efficient machining of advanced composites. Due to very high operating frequency of at least $20 \mathrm{kHz}$ of ultrasonic machining system, horn may be subjected to high stresses leading to failure. Mechanical horn is designed to get optimum vibration amplification while keeping stresses in acceptable limits. In this research, state of the art ultrasonic horns were designed with same length and diameters at the transducer side and tool ends under similar operating conditions. All standard and hybrid ultrasonic horns, including some new designs, suitable for machining applications were evaluated through finite element analysis. Modal analysis was performed for computing axial modal frequencies, whereas harmonic analysis was carried out to determine vibration amplitude, stresses and factor of safety. The performance of state of the art ultrasonic horn designs were later compared in terms of vibration amplification, stresses and operating life. The axial modal frequency and amplitude of vibration achieved by barrel, cylindrical-double conical and hollow exponential horns were observed to be greater as compared to the step horn, however the former were prone to greater stress concentrations and low operating life. Reasonably higher vibration amplification, factor of safety and low stresses were achieved by Bezier, cylindricalcatenoidal, cylindrical-Bezier, step-conical, step-catenoidal, step-Bezier, double conical, multistep and multistep-conical horn designs. Remarkably, circular hollow exponential and multistep-conical ultrasonic horns were observed to achieve vibration amplification, factor of safety and operating life higher than that of commercially available step horn.
\end{abstract}

Keywords ultrasonic horn design, finite element analysis, hybrid horns, harmonic analysis, modal analysis, magnification, stresses.

\section{Introduction}

Nomex honeycomb is an advanced soft and brittle composite made of phenolic resin reinforced by aramid fiber. It has extraordinary mechanical and thermal properties such as high compressive strength, ultra-lightweight, good flame retardation and better thermal insulation etc., due to which it is used in aerospace sandwich structures [1,2]. Owing to its soft and brittle nature, conventional machining such as orthogonal cutting, drilling, grinding and milling may cause poor machining quality, surface roughness, surface damage, delamination, burr formation and tearing of walls. To deal with such problems related to machining quality, ultrasonic assisted machining is found to be more suitable for the machining of soft and brittle composites [3]. The ultrasonic vibration assisted machining system (Fig. 1) [4, 5] consists of ultrasonic generator, piezoelectric transducer, mechanical horn and cutting tool. The ultrasonic generator converts low frequency (50-60 Hz) signal to high frequency (more than $20 \mathrm{kHz}$ ) electrical signal. The high frequency electrical signal is then converted to high frequency mechanical vibrations by the transducer. The vibration amplitude transmitted by transducer is usually very low (nearly $5 \mu \mathrm{m}$ ) and non-suitable for machining [6]. Therefore, a mechanical horn is required between the transducer and the cutting tool to enhance the amplitude of vibration. Reasonably good machining quality and reduction of forces have been achieved through ultrasonic machining technology [5, 7-9].

The ultrasonic horn design for particular machining application is quite challenging. Horn profile, material and other geometric parameters are usually decided based on the requirement of vibration amplitude suitable for ultrasonic machining of a particular material [10]. For this purpose several horn profiles such as cylindrical, conical, step, catenoidal, Gaussian, exponential and Bezier, have been designed and analyzed by researchers [10-15]. For highest 
vibration amplification, step horn is used by designers, researchers and industries, however at the expense greatest stress concentration due to sudden change in geometry resulting in reduced factor of safety [6, 13, 16-24]. $3^{\text {rd }}$ order Bezier horn has been suggested by designers for higher vibration amplification as compared to other standard ultrasonic horn designs except step horn, and low stresses due to smooth variation in cross sectional area [12, 25]. Stress concentrations can be reduced by incorporating smooth curves at the location of abrupt change in cross section [19, 26-30]. However it may cause reduction in amplitude of vibration as well [7]. The stresses in cylindrical, conical, Gaussian, exponential and catenoidal horn designs are very low, but the amplitude of vibration at the tool end is also extremely less as compared to the step design $[6,10,13,20,31,32]$. Slots and holes have been incorporated in some horn designs to increase the amplitude of vibration and reduce stresses [7, 26, 33-40]. The lengths and diameters of the cylindrical portions, cross sectional shape (circular to rectangular) [41] and the location of mountings has also been varied to optimize and improve the performance of ultrasonic horn [42-44]. Hybrid horns, which are combination of basic horn profiles such as multi-step [45, 46], cylindrical-conical / step-conical [31, 36, 39, 43, 44, 47-52], double conical [6, 53], step-exponential / cylindrical-exponential [43, 44, 53-55], multistep conical [56, 57], step-catenoidal [54], step-Gaussian / cylindrical -Gaussian [58] and cylindrical-conical-exponential [59] have also been designed and analyze to assess horn performance. Other horn designs have also been attempted in order to improve the amplitude of vibration while keeping the stresses in acceptable limits [33, 60-64]. The details of standard and hybrid horn designs along with their, geometry, advantages and limitations have been presented in upcoming sections. There is plenty of room to design and optimize an ultrasonic horn with greater vibration amplification but minimum stresses and more operating life.

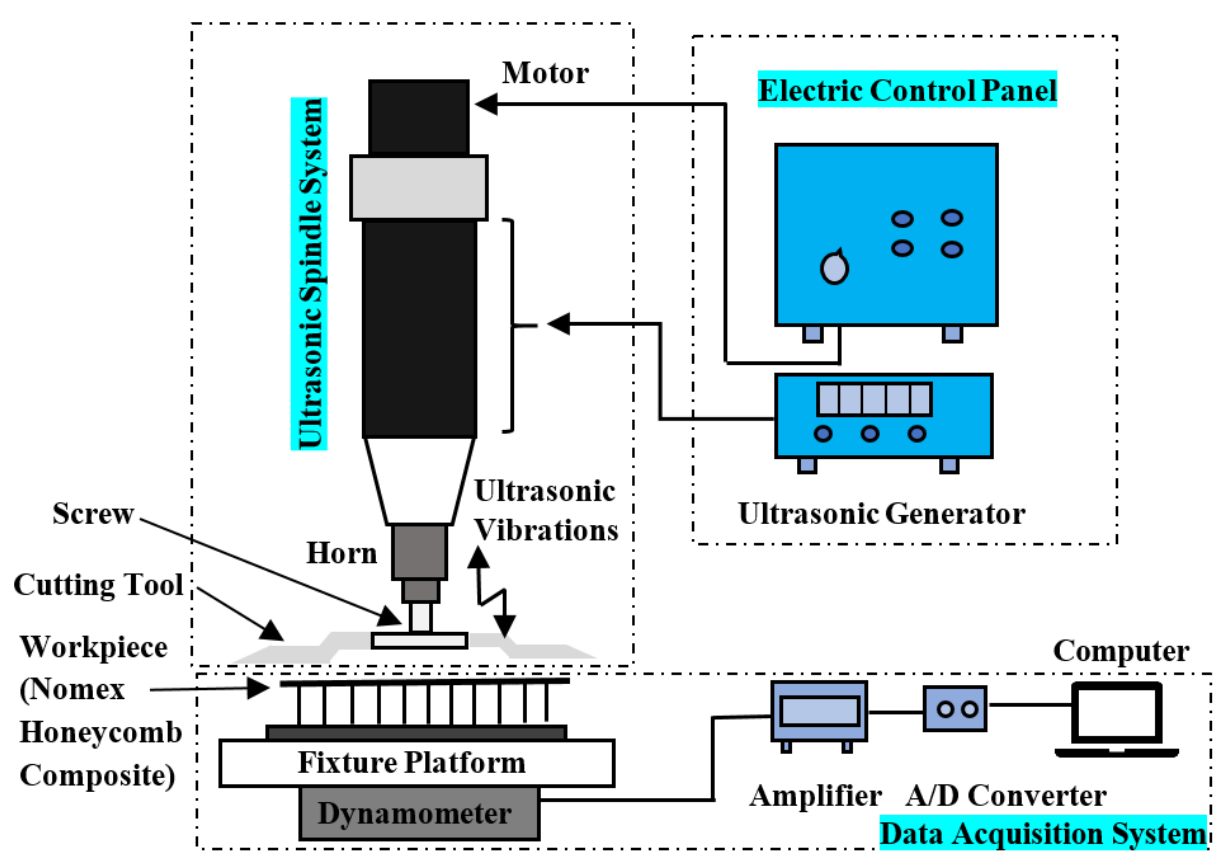

Fig. 1. Schematic of Ultrasonic Assisted Cutting System

The aim of this research was to evaluate state of the art ultrasonic horn designs available in literature that can be used for the machining of soft and brittle composites such as Nomex honeycomb core material. In the past research, various horn designs have been suggested and analyzed, but the dimensions and sizes were taken to be different for each application. Further, the performance comparison of some new designs were only made with few of the standard ultrasonic horns. There is a dire need to compare the performance of existing ultrasonic horn designs with comparable sizes under similar operating conditions. The current research filled this gap by analyzing and evaluating the performance of state of the art ultrasonic horns either standard or hybrid, with exactly same overall length, diameters at the transducer side and tool end and generator's operating frequency. With existing combinations, some new combinations of standard profiles were also designed and analyzed. This included integration of Guassian, Bezier, catenoidal and exponential profiles with cylindrical and stepped horn designs, whose performance in terms of modal frequency, vibration amplification, stresses and factor of safety have never been investigated in the past research. Finally, the ultrasonic horns were identified which provided larger vibration amplification, low stresses and more 
operating life. Vibration analysis of non-uniform horns is extremely difficult theoretically, and experimentations would involve lot of time and money, therefore an attempt was made to investigate complex horn designs through numerical computations. The outcomes of this research would be beneficial for the designers, researchers, scientists and manufacturers of the ultrasonic machining technology to select better ultrasonic horn designs for a particular material based on their requirement.

\section{State of the art ultrasonic horn designs}

\subsection{Standard ultrasonic horns}

The state of the art standard ultrasonic horns, designed and analyzed by the researchers, include conical, cylindrical, step, catenoidal, exponential, Gaussian, $2^{\text {nd }}$ order and $3^{\text {rd }}$ order Bezier. Each standard horn has its own merits and demerits which has been fully explored in the past research $[12,15]$. The standard ultrasonic horns considered in this research along with their geometries, mathematical models, advantages and limitations are presented in Table 1.

\subsubsection{Cylindrical horn}

Cylindrical shape is the most basic form of the ultrasonic horn. It has uniform cross sectional area and is easy to design and fabricate. It is used in ultrasonic machining systems as a whole or as a subcomponent of modern mechanical horns. The stress distribution and energy transfer are uniform, but the axial modal frequency and amplitude of vibration are low for cylindrical horns[15]. Analytical solutions are available to analyze the performance of the cylindrical horn. It is commonly used in ultrasonic machining, welding, cleaning and other industrial applications.

Xiping et al. has designed a cylindrical horn with cuboid hole near the tool end, to increase the amplitude of vibration. He optimized the size and placement of the cuboid hole in terms of horn diameter for higher vibration amplification [38]. Rani et al. developed a tubular horn with slits for ultrasonic welding. They optimized the hole diameter, tube thickness, and number, size and placement of slits in terms of horn length and diameter for better performance [26]. Xiping et al. investigated the effect of bend angle on the performance of the ultrasonic horn used in applications such as ultrasonic systems for bone cutting, ultrasonic-assisted turning of deep concave work-piece and ultrasonic cleaning of internal surface of a bent pipe [65]. Amini et al. conducted finite element analysis on cylindrical horns with eccentric and concentric tool inserts respectively, for ultrasonic vibration assisted turning applications [31].

\subsubsection{Conical horn}

This ultrasonic horn is simple to design and fabricate. The stresses are uniformly distributed due to gradual decrease in the cross sectional area along the straight line. The vibration amplification achieved through conical horn is very low, however greater as compared to that of cylindrical horn. Mathematical expressions are available in literature to analyze the vibrations of the conical horn. It is used, as a single unit or subcomponent, in various ultrasonic applications such as machining, welding and medical applications, where high forces and energy transfer are required at output.

\subsubsection{Stepped horn}

It is the most commonly used horn type used in ultrasonic applications. It provides the greatest vibration amplification which can be calculated by $(D / d)^{2}$. However, the energy losses and stress concentrations are greatest at the location of abrupt change in cross sectional area. Although, the stress concentrations can be reduced to some extent by incorporating fillet at step location [53], however increasing the fillet radius reduces the amplitude of vibration at tool end. Since stresses produced in ultrasonic horn are dynamic in nature, therefore they do not depend only on the cross sectional properties. They depend on axial modal frequency, generator frequency, mass and geometry of horn as well.

Pang et al. produced diagonal slits / spiral slots in the step profile to enhance the performance of the ultrasonic machining system. They analyzed the influence of step and slot location with a spiral angle of $52^{\circ}$ on the modal frequency and vibration amplitude of ultrasonic horn. They achieved significant reduction in cutting forces and improvement in surface quality through coupling of longitudinal and torsional modes of vibration [7]. Lin et al. proposed and studied an actively adjustable horn composed of step profile and piezoelectric material, connected to an 
adjustable electrical impedance. They investigated the influence of varying the electric impedance and location of piezoelectric material on the resonance frequency and vibration amplitude of the ultrasonic horn. They showed that the resonance frequency and the amplitude of vibration will be larger if the piezoelectric material is placed near the transducer end of ultrasonic step horn [21].

\subsubsection{Catenoidal / Catenary horn}

It is also called as catenary horn. The profile of catenoidal horn is based on the cosine hyperbolic function. It provides low to moderate vibration amplification, and stresses are uniformly distributed due to smooth variation of the cross sectional area. This horn was used in ultrasonic applications, where larger forces, low vibration amplification and reasonably higher operating life were desired $[10,15]$. Various researchers used the catenoidal profile as a subcomponent of the composite horn to enhance the performance of the ultrasonic system [54]. The mathematical model of the catenoidal horn is complex, therefore vibration analysis is difficult to perform analytically. Therefore, numerical methods are used to compute resonant frequency, amplitude of vibration and stresses.

\subsubsection{Exponential horn}

Exponential horns are based on exponentially decaying mathematical function[10, 66]. Exponentially decreasing cross sectional area results in smooth distribution of stress over entire horn length eliminating stress concentrations. However, the amplitude of vibration is low as compared to other standard horns [13, 20, 59]. Good efficiency and low energy losses can be achieved at the tool end through such horns. Exponential horns are commonly used in ultrasonic applications where high forces and low vibration amplification are desired at tool end. Due to complex geometry, exponential horns can be designed and analyzed through numerical methods [46].

Generally, the ultrasonic horn used for research and industrial applications have circular cross section for smooth connection and energy transfer. Jagadish et al analyzed the influence of rectangular cross section on the performance of exponential horn and compared with that of circular cross section [41]. Researchers has integrated the exponential profile with cylindrical, step and conical profiles and investigated the performance of ultrasonic system $[41,43,53$ $55,59]$.

\subsubsection{Gaussian horn}

Gaussian horn has low to moderate vibration amplification, albeit higher magnification as compared to exponential and catenoidal horns. The stress distribution is smooth due to gradual variation of the cross sectional area eliminating stress concentrations $[15,58]$. Due to complex mathematical model, numerical methods are used to evaluate the performance of Gaussian horn [12].

\subsubsection{Bezier horn}

Bezier horn is the most versatile horn among all other standard ultrasonic horns. Infinite horn profiles can be designed for unique values of diameters at the transducer and tool ends. General mathematical forms for Bezier curves are available in literature. Designer can decide the location of control points to define unique Bezier curve. Bezier curve is based on parametric polynomial equations. The number of control points required to define the curve is equal to order of polynomial plus one. For example, third order Bezier curve needs four control points. In case of ultrasonic applications, two control points are defined by horn diameters at the transducer and tool ends. Two control points remain for optimization, which can be defined anywhere in the horn regime to get a third order Bezier profile for higher vibration amplification [25, 67]. Wang et al designed and optimized third order Bezier horn for ultrasonic welding. They found that the amplitude of vibration achieved by Bezier horn is larger as compared to that of catenoidal horn, but lesser as compared to step horn. Albeit, the stresses are found lesser as compared to step and catenoidal designs [12]. Rani et al investigated the dynamic performance of standard ultrasonic horns used in plastic welding. They observed the amplitude of vibration achieved by Bezier horn to be closer to step horn and greater as compared to cylindrical, Gaussian and catenoidal horns. Albiet the stresses are closer to step horn and much larger as compared to other horn designs considered [15]. This prompted the need of a novel horn whose performance is close to step horn in terms of vibration amplification while keeping stresses as much lesser as possible. Kumar et al. designed and optimized $2^{\text {nd }}$ and $3^{\text {rd }}$ order Bezier horns for rotary ultrasonic machining applications and observed the performance 
of third order Bezier horn to be beetr as compared to second order Bezier horn in terms of vibration amplitude and stresses [25].

Table 1. Standard ultrasonic horns, geometrical parameters, mathematical models, advantages and limitations.

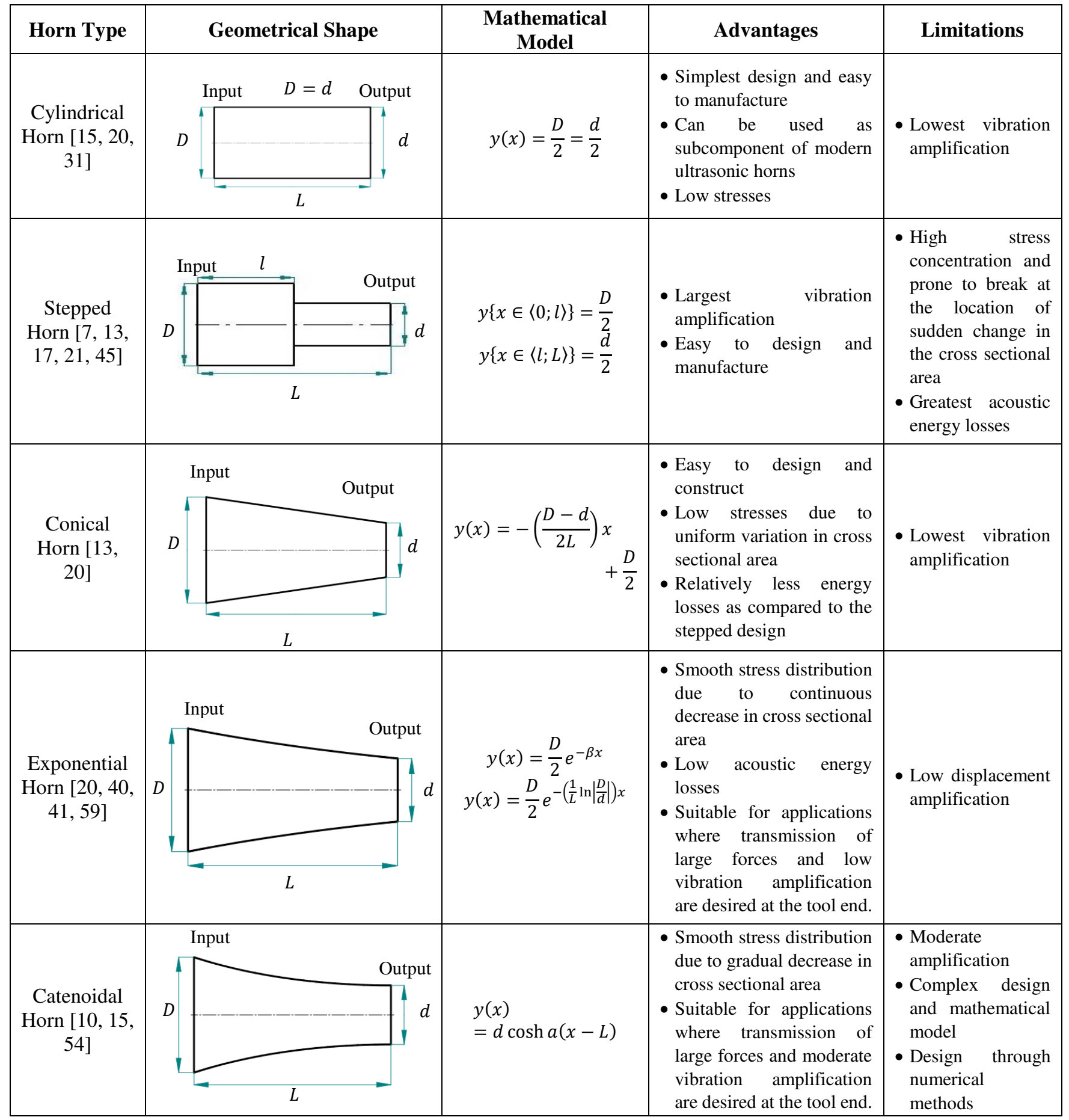




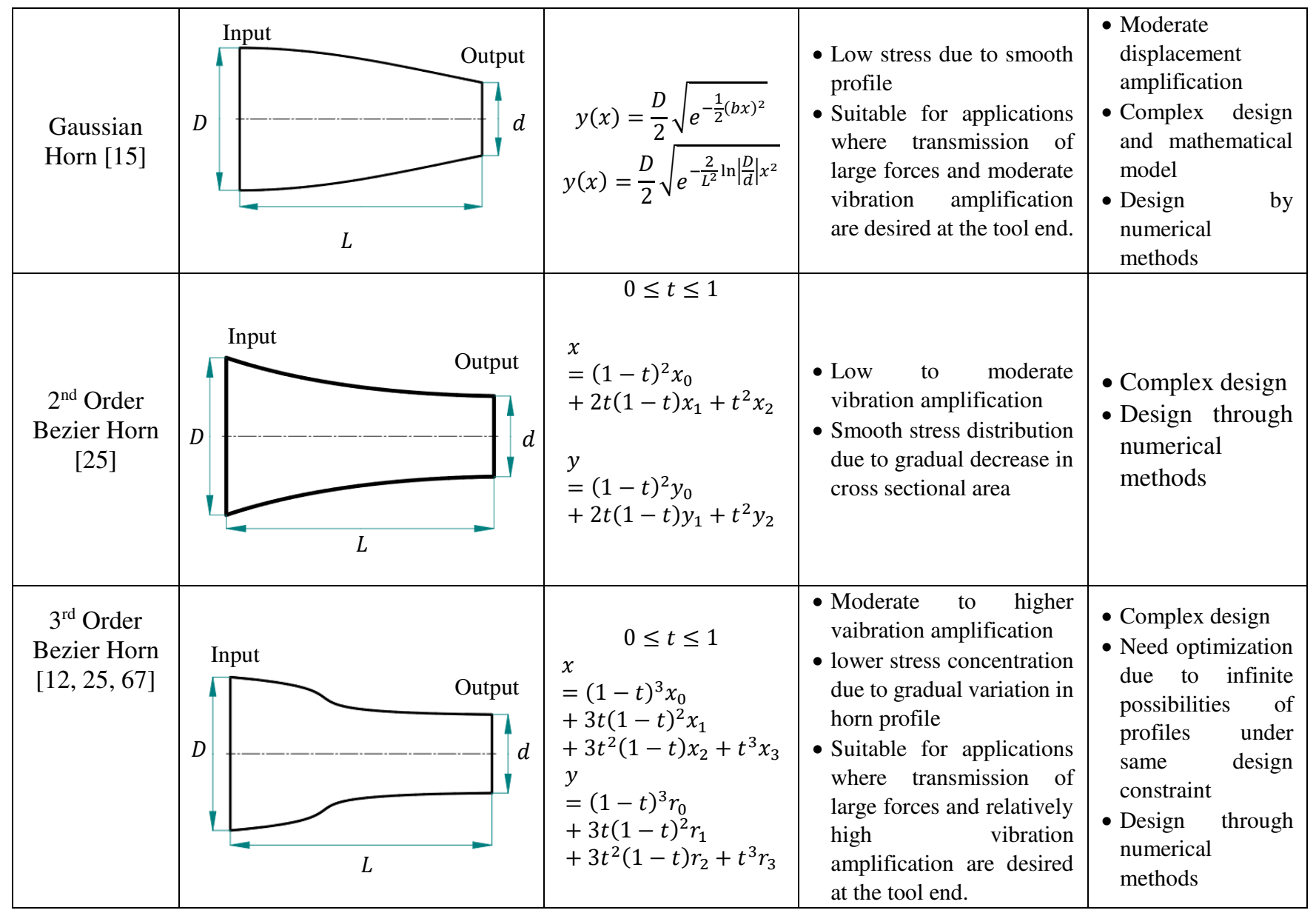

\subsection{Hybrid horns}

Hybrid horns or composites horns are the combination of the standard ultrasonic horn profiles. Since step horn provides greatest vibration amplification albeit at the expense of greatest stress concentration, therefore researchers has integrated basic horn geometries to develop hybrid horns to achieve higher vibration amplification and low stress concentrations [28]. The hybrid ultrasonic horns explored by researchers are presented in the following sections.

\subsubsection{Hybrid cylindrical horn}

These horns were developed by integrating the cylindrical horn with one of the standard horn profiles such as conical, catenoidal, exponential, Gaussian and Bezier. The hybrid cylindrical horns considered in this research along with their geometries, anticipated advantages and limitations are presented in Table 2. The influence of placing cylindrical portion at transducer side (larger end) and other standard profiles at tool end (smaller end), and standard profile at transducer side and cylindrical portion at tool end, was also investigated, which have not been explored in the past research.

\subsubsection{Cylindrical-conical horn}

This ultrasonic horn is a combination of cylindrical and conical profiles. In this horn type, one cylindrical portion of the step horn is replaced by conical section, which helps in eliminating the stress concentration. However, the amplitude of vibration greatly reduces at the tool end. Researchers have investigated the performance of both cylindrical-conical (conical portion at the tool side) and conical-cylindrical horn designs (conical portion at the transducer side), however cylindrical conical arrangement has been used in commercially available ultrasonic systems $[31,52,53]$. 


\subsubsection{Cylindrical-catenoidal horn}

This ultrasonic horn is the combination of cylindrical section at the transducer side and catenoidal section near the tool side. Effect of integrating cylindrical section and catenoidal curve has not been investigated in the past research.

\subsubsection{Catenoidal-cylindrical horn}

This ultrasonic horn is made by placing the catenoidal profile at the transducer side while cylindrical portion at the tool side. Effect of integrating catenoidal horn with cylindrical horn has not been investigated in the past research.

\subsubsection{Cylindrical-exponential horn}

This ultrasonic horn is the combination of cylindrical section followed by an exponential section. Wang et al designed cylindrical-double exponential horn to be used in ultrasonic system for microelectronics packaging, which consisted of cylindrical portion joined by two exponential curves at the tool end. They investigated the performance of hybrid horn using finite element method. The effect of using rectangular cross section was also investigated and the results were compared with horn having circular cross section. The performance of ultrasonic horn with cross section was observed to be better as compared to that having circular rectangular cross section in terms of vibration amplification and stresses. Jagadish et al also investigated the performance of cylindrical-exponential horn with rectangular cross section and observed the performance better as compared to similar design with circular cross section [41]. Since the cross section of transducers and ultrasonic tool are circular, therefore rectangular horns are not preferred for smooth transfer of ultrasonic energy.

Exponential-cylindrical horn

This ultrasonic horn can be developed by integrating exponential curve at the transducer side with cylindrical portion at the tool side. Performance of exponential-cylindrical horn has not been investigated in the past research.

Table 2. Cylindrical hybrid ultrasonic horns, geometrical parameters, advantages and limitations.

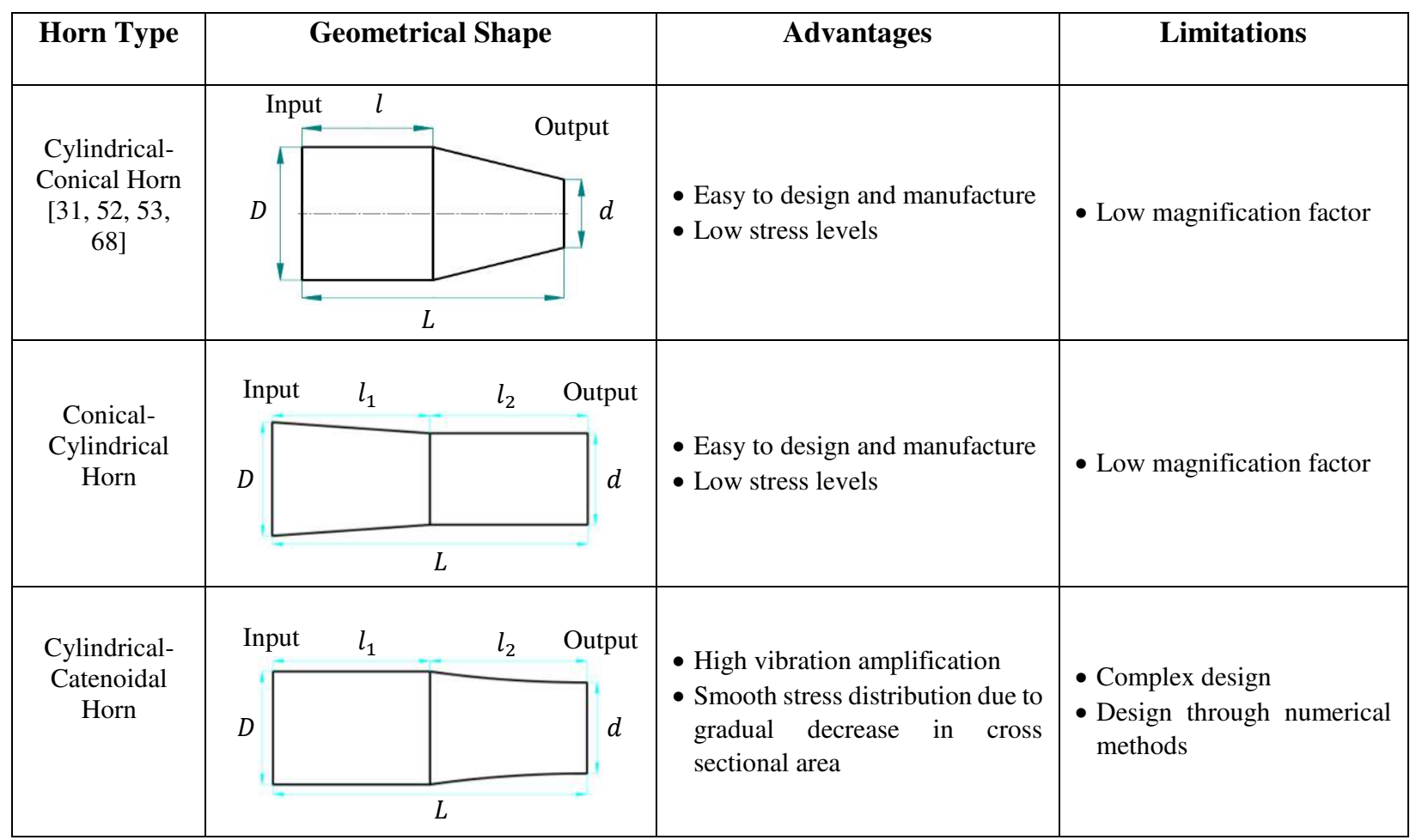




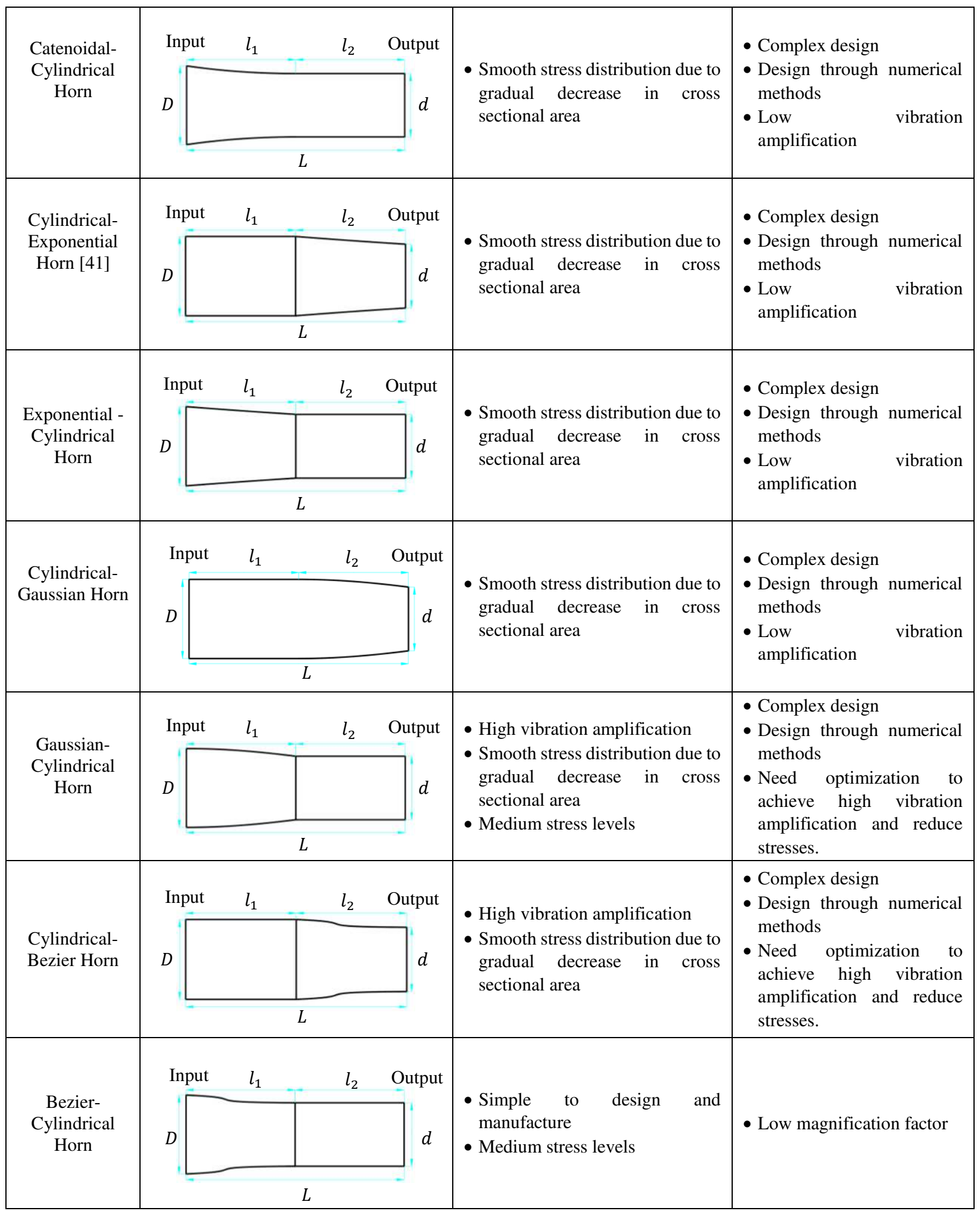




\subsubsection{Cylindrical-Gaussian horn}

This ultrasonic horn can be designed by integrating cylindrical horn with Gaussian horn. The cylindrical portion is placed at transducer side whereas the Gaussian profile is used near the tool end to develop such ultrasonic horn. The influence of integrating conical section with Gaussian curve has not been investigated in the past research.

\subsubsection{Cylindrical-Bezier horn}

The cylindrical horn can be combined with the Bezier horn to develop hybrid horn. Bezier horn has good ultrasonic performance in terms of vibration amplification and stresses. Bezier horn has never been integrated with any standard ultrasonic horn in the past research. In this paper, Bezier horn was integrated with cylindrical horn and the horn performance was examined.

\subsubsection{Bezier-cylindrical horn}

The Bezier profile can be integrated with the cylindrical profile to generate Bezier-cylindrical horn. In this research, the Bezier profile was used at the transducer side, while the cylindrical portion was used near the tool end of horn. The performance of Bezier-cylindrical horn was analyzed in terms of vibration amplification and stresses, which has not been investigated in the past research.

\subsubsection{Hybrid step horns}

These horns were developed by incorporating standard ultrasonic horn profile such as conical, exponential, catenoidal, Gaussian and Bezier respectively, into step design usually at the location of abrupt change in cross sectional area. The purpose was to reduce stress concentrations in step design by incorporating smooth line or curve between two cylindrical portions. The step hybrid ultrasonic horn designs along with geometric parameters, anticipated advantages and limitations are presented in Table 3.

Table 3. Step hybrid ultrasonic horns, geometrical parameters, advantages and limitations.

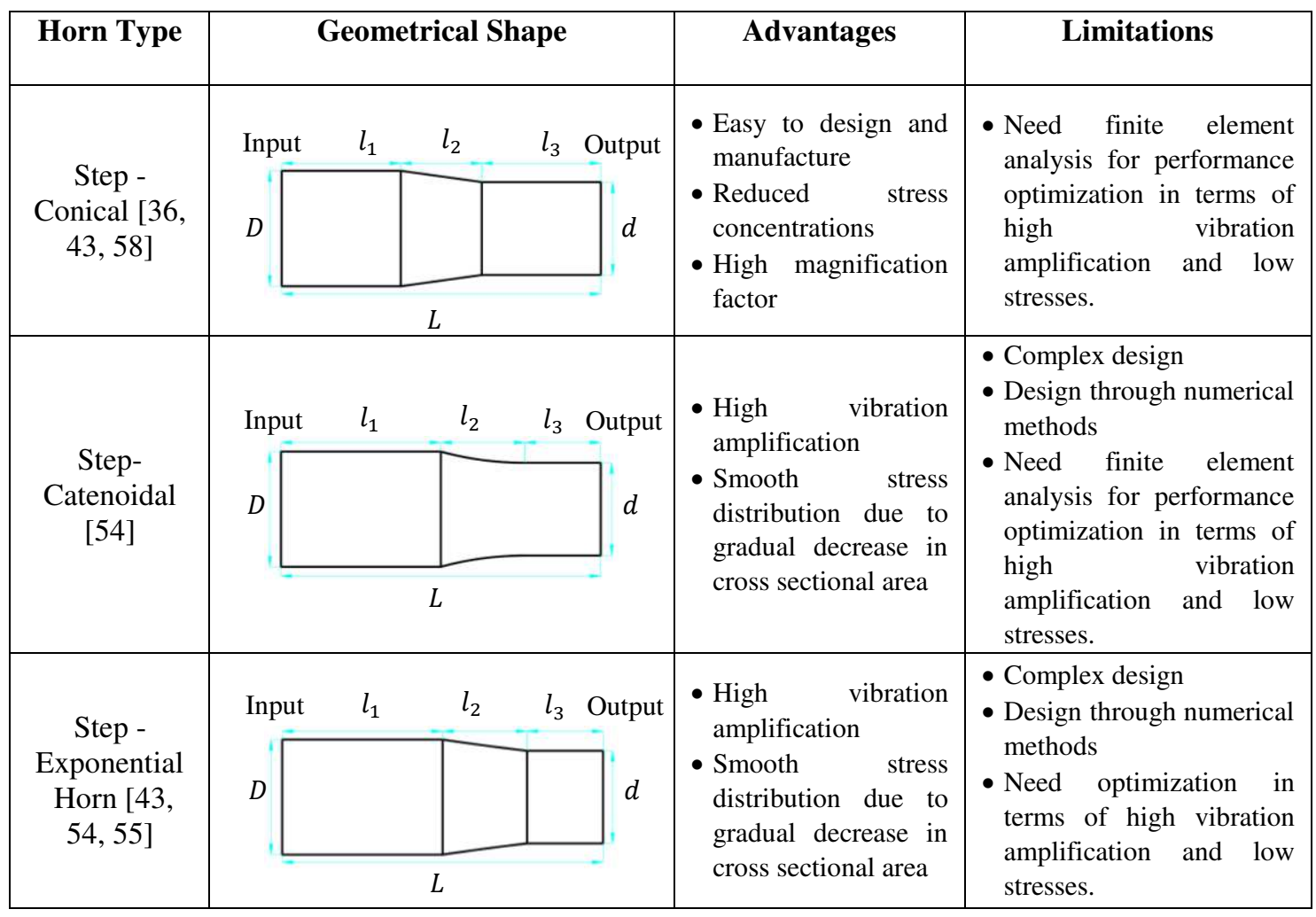




\begin{tabular}{|c|c|c|c|c|c|c|}
\hline $\begin{array}{c}\text { Step - } \\
\text { Gaussian } \\
\text { Horn [58] }\end{array}$ & Input & $l_{1}$ & $l_{3}$ & Output & $\begin{array}{l}\text { - High vibration } \\
\text { amplification } \\
\text { - Smooth stress } \\
\text { distribution due to } \\
\text { gradual decrease in } \\
\text { cross sectional area }\end{array}$ & $\begin{array}{l}\text { - Complex design } \\
\text { - Design through numerical } \\
\text { methods } \\
\text { - Need optimization in } \\
\text { terms of high vibration } \\
\text { amplification and low } \\
\text { stresses. }\end{array}$ \\
\hline $\begin{array}{l}\text { Step - Bezier } \\
\text { Horn }\end{array}$ & Input & $l_{1}$ & $l_{3}$ & Output & $\begin{array}{l}\text { - High vibration } \\
\text { amplification } \\
\text { - Smooth stress } \\
\text { distribution due to } \\
\text { gradual decrease in } \\
\text { cross sectional area }\end{array}$ & $\begin{array}{l}\text { - Complex design } \\
\text { - Design through numerical } \\
\text { methods } \\
\text { - Need optimization in } \\
\text { terms of high vibration } \\
\text { amplification and low } \\
\text { stresses. }\end{array}$ \\
\hline $\begin{array}{l}\text { Step-Conical } \\
{[47]}\end{array}$ & $\begin{array}{c}\text { Input } l \\
D \\
D\end{array}$ & $l_{2}$ & $l_{3}$ & Output & $\begin{array}{l}\text { - Easy to design and } \\
\text { manufacture }\end{array}$ & $\begin{array}{l}\text { - Low magnification factor } \\
\text { - Low stress levels }\end{array}$ \\
\hline
\end{tabular}

\subsubsection{Step-conical horn}

Researchers have also replaced the abrupt change in cross section with curves to reduce the stress concentrations in step horn. In step-conical design, the conical section is inserted at step location between the two cylindrical portions of the step horn [58]. In addition to reduce stress concentration, it helps in achieving higher vibration amplification at the tool end [36]. However, the amplitude of vibration attained by step-conical horn was found to be lesser as compared to that of step-exponential horn [43]. Ma Ke et al designed and analyzed step-conical horn by incorporating conical section at the end of step horn design for ultrasonic machining of Nomex honeycomb composite [47].

\subsubsection{Step-catenoidal horn}

In this horn design, catenoidal section is placed between the two cylindrical portions of the step horn. The effect of varying the length of the catenoidal section in step-catenoidal design was investigated by Woo et al. The amplitude of vibration at the tool end was found to decrease with the increase of the length of catenoidal section. However, the reduction in vibration amplitude was observed to be lesser as compared to that of step-exponential horn. For shorter length of curved section, the amplitude of vibration for step-catenoidal horn was found to be lesser as compared to similar sized step-exponential horn. However, opposite was the case for longer curved section [54].

\subsubsection{Step-exponential horn}

This horn is developed by incorporating the exponential section at the step location between the two cylindrical sections of the step horn. Woo et al investigated the effect of varying the length of exponential section on the vibration characteristics of step-exponential horn design and found that the magnification factor decrease by increasing the length of exponential section. For shorter curve, the magnification factor achieved by step-exponential horn was found greater as compared to similar sized step-catenoidal horn [54]. Choi et al. designed step-exponential horn to investigate the effect of ultrasonic vibration in grinding [55]. Xiao et al conducted the research on ultra-long ultrasonic horn mainly used for machining deep hole parts. They performed modal and harmonic analysis on the step-exponential and step-conical horn designs to compute modal frequencies and amplitude of vibration. They observed the performance of step-exponential horn to be better as compared to its conical counterpart in terms of resonant frequency and vibration amplification [43]. 


\subsubsection{Step-Gaussian horn}

This horn is developed by incorporating Gaussian profile at the location of varying cross sectional area, i.e. two cylindrical portions in step horn design. Tang et al. incorporated Gaussian profile in step horn design at transition section and compared the performance with step-conical horn design [58].

\subsubsection{Step-Bezier horn}

This horn is developed by incorporating $3^{\text {rd }}$ order Bezier profile at the location of varying cross sectional area i.e. two cylindrical portions in step horn design. The effect of integrating Bezier curve into step horn design has not been investigated in the past research.

\subsubsection{Specialized complex hybrid ultrasonic horns}

Researchers have also investigated the performance of some specialized hybrid ultrasonic horns which are combinations of multiple standard horn profiles or involve some unique geometric feature, for higher vibration amplification. The complex hybrid ultrasonic horn designs considered in the past research along with their geometric parameters, anticipated advantages and limitations are presented in Table 4.

\subsubsection{Cylindrical horn with cuboid hole}

The cylindrical horn has lowest vibration amplification among all standard ultrasonic horns. Xiping et al. designed a cylindrical horn with cuboid hole near the tool end to achieve higher amplitude of vibration for improving work efficiency [38]. They optimized the geometrical size and position of the cuboid hole and studied the influence of these parameters on resonant frequency and hence vibration amplification achieved by ultrasonic horn.

\subsubsection{Multistep horn}

The stress concentration in step horn depends on the diameter ratio at the changing cross section. One method to reduce the stress concentration is to design multi step horn with more than two cylindrical portions resulting in reduction of the diameter ratio. Since the vibration amplification achieved by step horn is equal to square of the ratio of diameter of cylindrical portions, therefore vibration amplification is also reduced by increasing the number of steps [46]. Naseri et al used multi step horn design for ultrasonic vibration-aided equal channel angular pressing [45]. In this research, the performance of multistep ultrasonic horn was investigated for ultrasonic vibration assisted machining applications for soft and brittle composites.

\subsubsection{Double conical horn}

This ultrasonic horn design involved two conical sections [6]. Amin et al designed new double conical horn using finite element analysis for ultrasonic machining of hard and strong materials. They optimized the horn profile to achieve maximum amplification possible for safe working stresses and high material removal rates. Yadava et al. designed double conical horn with hole at the tip for cooling, using finite element analysis to be used in rotary ultrasonic machining applications [49]. They analyzed various components of stresses and found stresses to be maximum at the middle of horn but were observed to be in acceptable limits. They also analyzed stresses in the horns at various frequencies.

\subsubsection{Cylindrical-conical-exponential horn}

This ultrasonic horn is combination of cylindrical, conical and exponential profiles. Wang et al designed this novel ultrasonic horn using finite element methods for microelectronics packaging applications [59].

\subsubsection{Cylindrical-double exponential horn}

This ultrasonic horn is combination of cylindrical and two exponential profiles. Wang et al designed this novel ultrasonic horn using finite element methods for microelectronics packaging applications by replacing the conical 
section in cylindrical-conical exponential design with exponential profile [59]. They found that replacing conical section with exponential profile in their novel designs had not improved the horn performance in terms vibration implication, rather added complexity.

\subsubsection{Barrel horn}

This five element novel ultrasonic horn was designed and analyzed by Peshkovsky et al. for ultrasonic application related to matching transducer to water at cavitation [48]. This horns consists of three cylindrical portions separated by two conical portions individually. The diameter of the central cylinder was taken larger than that of the cylindrical portions at the transducer and tool sides.

\subsubsection{Barbell horn}

This five element novel ultrasonic horn was designed and analyzed by Peshkovsky et al. for ultrasonic application related to matching transducer to water at cavitation [48]. This horns consists of three cylindrical portions separated by two conical portions individually. The diameter of the central cylinder was taken smaller than that of the cylindrical portions at the transducer and tool sides. Fu et al. also analyzed barbell horn using finite element methods and proposed optimized design to achieve largest vibration amplification possible for ultrasonic liquid processing applications [50]

\subsubsection{Spool horn}

This five element novel ultrasonic horn was designed and analyzed by Peshkovsky et al. for ultrasonic application related to matching transducer to water at cavitation [48]. This horns consists of three cylindrical portions separated by two conical portions individually. The diameter of the central cylinder was taken smaller than that of the cylindrical portions at the transducer and tool sides, while the lengths of the conical portions were set larger than that of cylindrical portions.

Table 4. Specialized complex hybrid ultrasonic horn designs, geometrical parameters, advantages and limitations.

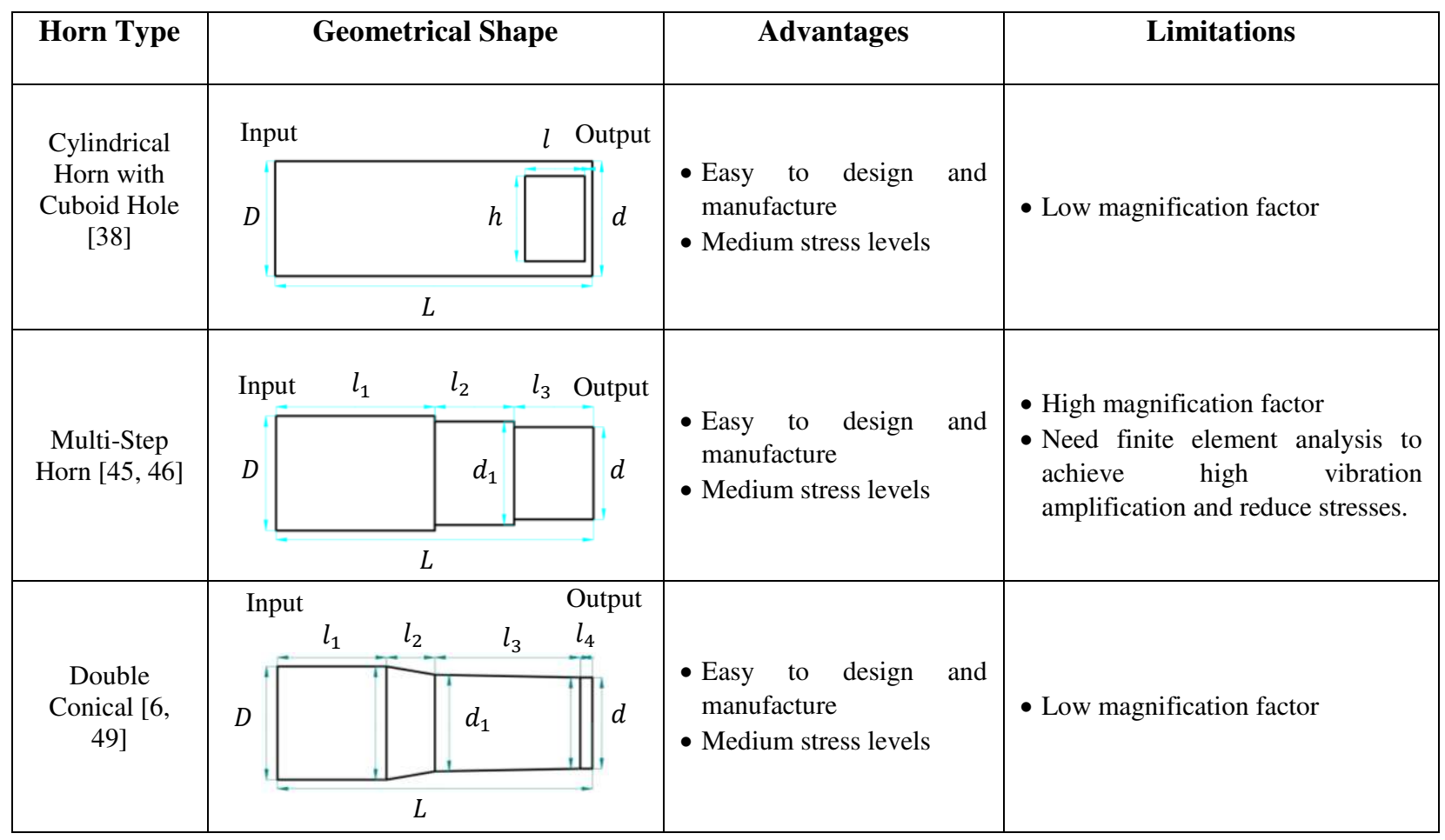




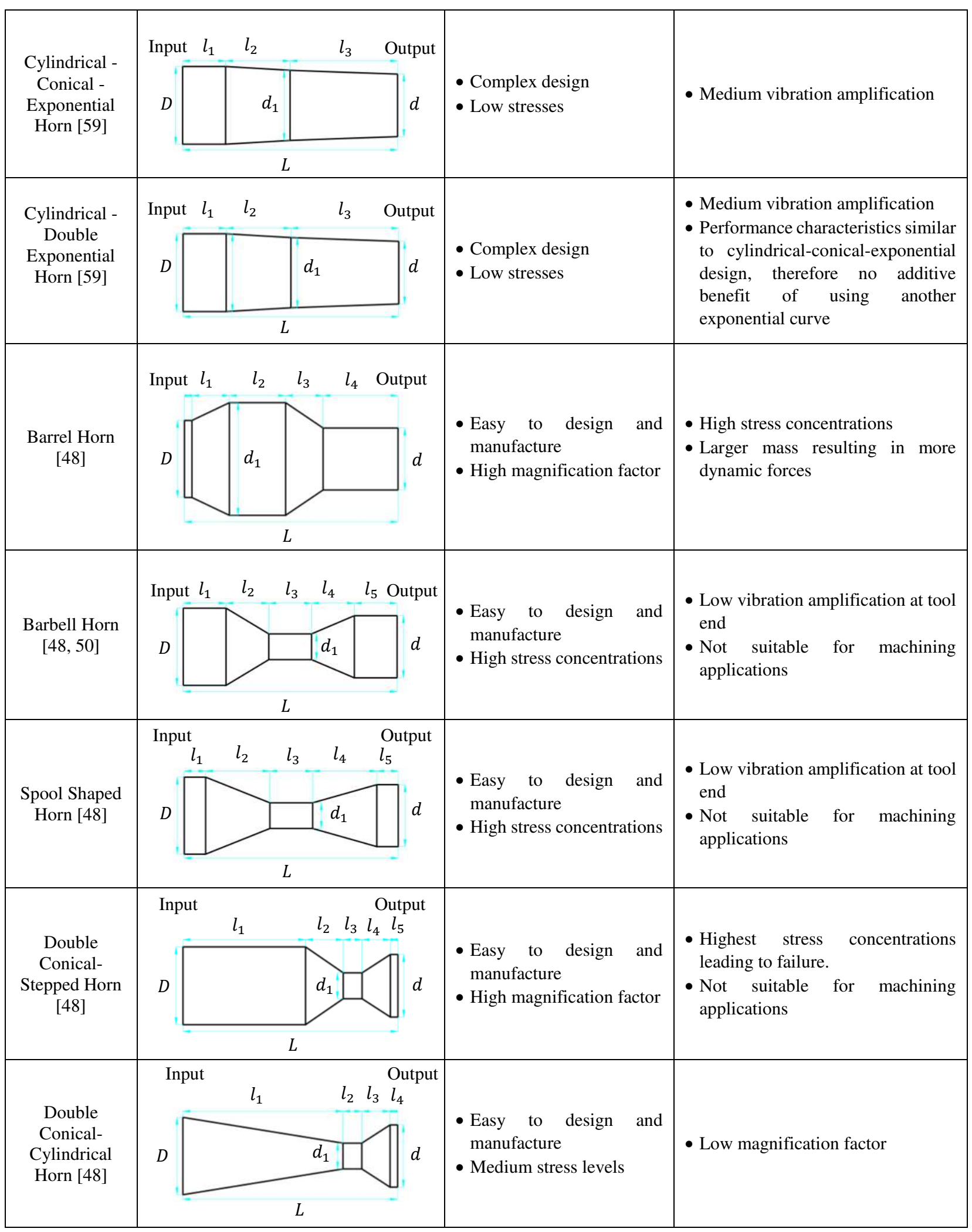




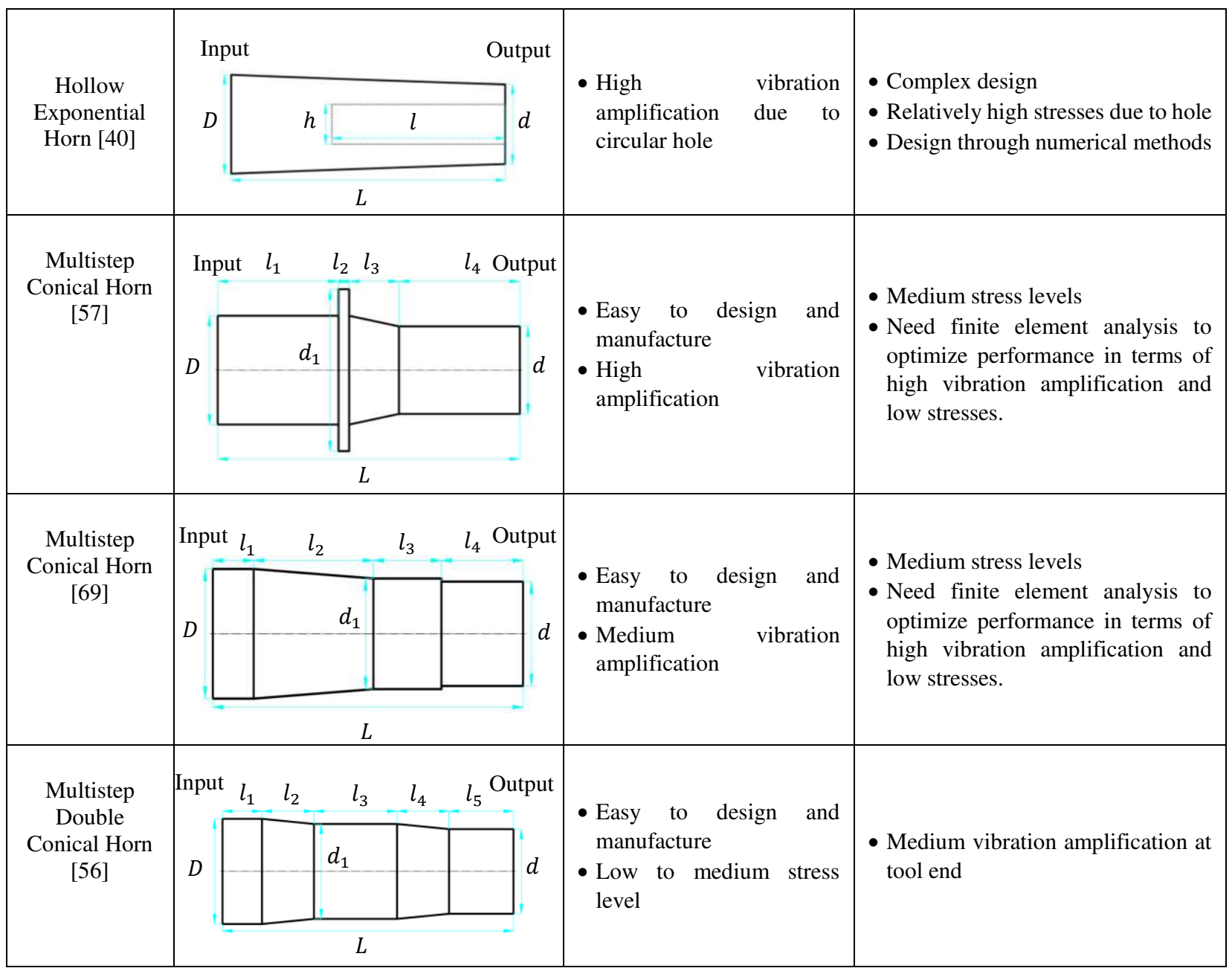

\subsubsection{Step-double conical horn}

This five element novel ultrasonic horn was designed and analyzed by Peshkovsky et al. for ultrasonic application related to matching transducer to water at cavitation [48]. This horns consists of three cylindrical portions separated by two conical portions individually. The diameter of the central cylinder was taken smaller than that of the cylindrical portions at the transducer and tool sides. The cylinder length at the transducer side was set largest as compared to other horn components, while length of cylindrical portion near the tool end was taken smallest.

\subsubsection{Cylindrical-double conical horn}

This four element novel ultrasonic horn was designed and analyzed by Peshkovsky et al. for ultrasonic application related to matching transducer to water at cavitation [48]. This horns consists of two conical portions separated by two cylindrical portions individually. The cone length at the transducer side was set largest as compared to other horn components, while length of cylindrical portion near the tool end was taken smallest. The diameter of the middle cylinder was taken smallest.

\subsubsection{Exponential hollow horn}

Roy et al designed exponential hollow ultrasonic horn for ultrasonic machining system using finite element analysis [40]. They used modal analysis to determine the mode shapes and associated modal frequencies, whereas harmonic 
analysis to determine magnification factor and equivalent stresses. They found the performance of exponential hollow horn to be superior than the conical and exponential horn in terms of vibration amplification and stresses.

\subsubsection{Multistep conical horn}

Kumar et al designed multistep conical ultrasonic horn for ultrasonic vibration assisted friction stir welding using finite element analysis [57]. They used modal analysis to determine the mode shapes and associated modal frequencies, whereas harmonic analysis to determine magnification factor and equivalent stresses. They found large gap between axial and non-axial modes of vibration for newly designed horn, eliminating the chance of modal decoupling. They also analyzed complex by attaching front part, a pair of bearings and nut and bolt assembly to the multistep conical horn. The modal frequency and amplitude of vibration were found to be lesser than multistep conical horn. However, both designed were found to be safe within metallurgical and design limits. Zhang et al. designed and analyzed multistep conical horn for ultrasonic milling system, which was combination of multiple cylindrical sections and one conical section located between the first two cylinders [69].

\subsubsection{Multistep double conical horn}

Razavi et al designed and analyzed multistep double conical, also termed as cylindrical-conical five element horn through finite element analysis and validated the numerical results through experimentations [56]. They developed it by modifying the design of multistep horn and incorporation conical profiles at the transition sections for ultrasonic assisted surface rolling process.

\section{Materials and methods}

\subsection{Problem formulation and governing equations}

The propagation of acoustic longitudinal waves in the ultrasonic horn is expressed by Webster's horn equation / wave equation in the form [70,71].

$$
\frac{\partial^{2} u(x, t)}{\partial x^{2}}+\frac{\partial^{2} u(x, t)}{\partial x^{2}} \frac{\partial}{\partial x} \ln A(x)=\frac{1}{c^{2}} \frac{\partial^{2} u(x, t)}{\partial t^{2}}
$$

Here $c=\sqrt{E / \rho}$ is the propagation velocity of longitudinal wave in horn material. For simple harmonic motion equation for ultrasonic horn can be expressed as

$$
\begin{aligned}
& \frac{\partial^{2} u(x, t)}{\partial x^{2}}+\frac{\partial^{2} u(x, t)}{\partial x^{2}} \frac{\partial}{\partial x} \ln A(x)=\frac{\omega^{2}}{c^{2}} u(x, t) \\
& \frac{\partial^{2} u(x, t)}{\partial x^{2}}+\frac{\partial^{2} u(x, t)}{\partial x^{2}} \frac{\partial}{\partial x} \ln A(x)=k_{1}{ }^{2} u(x, t)
\end{aligned}
$$

$k_{1}=\omega / c$ is circular wavenumber. Solution of wave equation provides the displacement of ultrasonic horn at the desired position and time.

$$
u(x, t)=\frac{1}{\sqrt{A(x)}}\left(C_{1} \sin K_{1} x+C_{2} \cos K_{1} x\right)
$$

where $u(x, t)$ is the function of particle displacement in terms of the coordinate in the longitudinal direction $x$ and time $t, k_{1}$ is the wave number, $A(x)$ is the area of cross-section, $C_{1} \& C_{2}$ are variables depending on the initial conditions of the system, and $K_{1}$ is a variable depending on $k, A$ and $x$. The Von Mises stress can be calculated from (5) after computing principal stresses $\sigma_{1}, \sigma_{2}$ and $\sigma_{3}$ [72]. 


$$
\sigma_{V M}=\sqrt{\frac{\left[\left(\sigma_{1}-\sigma_{2}\right)^{2}+\left(\sigma_{2}-\sigma_{3}\right)^{2}+\left(\sigma_{3}-\sigma_{1}\right)^{2}\right]}{2}}
$$

The factor of safety for ultrasonic horn materials can be computed through (6) by using maximum Von Mises stress and yield stress of the material $\sigma_{Y}$.

$$
F S=\sigma_{V M} / \sigma_{Y}
$$

The magnification factor $M F$ for ultrasonic horn can be determined using (7) by taking the ratio of vibration amplitude $X_{2}$ at tool end to the displacement at the transducer end $X_{1}$.

$$
M F=X_{2} / X_{1}
$$

\subsection{Finite element analysis of ultrasonic horn}

Since the compound ultrasonic horn is a continuous system, there are infinite modes of vibrations, thus natural frequencies associated with ultrasonic horn. The natural frequencies of horn vibration can be computed numerical by using (8). Further, the damping has little effect on the resonant frequency, amplitude of vibration and stresses in ultrasonic horn at operating frequency of $20 \mathrm{kHz}$, therefore damping can be considered negligible for first longitudinal mode [20] and modeling of ultrasonic horn with undamped vibrating system would provide good results $[12,15,19$, $63,67]$.

$$
\left|[K]-\omega^{2}[M]\right|=0
$$

Strains can be determined by using (9) while stresses can be determined by using (10-11). Here $\{\epsilon\}$ is the strain matrix, $\{\sigma\}$ is the stress matrix, $[B]$ is the matrix of nodal coordinates, $\left\{d_{h}\right\}$ is matrix of nodal displacements and $\left[D_{h}\right]$ is matrix of horn material properties. Nodal displacements can be calculated from force, stiffness and displacement relation $\{F\}=[K]\left\{d_{h}\right\}$.

$$
\begin{aligned}
\{\epsilon\} & =[B]\left\{d_{h}\right\} \\
\{\sigma\} & =\left[D_{h}\right]\{\epsilon\}=\left[D_{h}\right][B]\left\{d_{h}\right\}
\end{aligned}
$$

$$
\left\{\begin{array}{c}
\sigma_{x} \\
\sigma_{y} \\
\sigma_{z} \\
\tau_{x y} \\
\tau_{y z} \\
\tau_{x z}
\end{array}\right\}=\frac{E}{(1+v)(1-2 v)}\left[\begin{array}{cccccc}
1-v & v & v & 0 & 0 & 0 \\
v & 1-v & v & 0 & 0 & 0 \\
v & v & 1-v & 0 & 0 & 0 \\
0 & 0 & 0 & \frac{1-2 v}{2} & 0 & 0 \\
0 & 0 & 0 & 0 & \frac{1-2 v}{2} & 0 \\
0 & 0 & 0 & 0 & 0 & \frac{1-2 v}{2}
\end{array}\right]\left\{\begin{array}{c}
\epsilon_{x} \\
\epsilon_{y} \\
\epsilon_{z} \\
\gamma_{x y} \\
\gamma_{y z} \\
\gamma_{x z}
\end{array}\right\}
$$

\subsection{Research methodology}

The primary objective of the current research was to evaluate state of the art ultrasonic horn designs comprehensively and critically in terms of vibration amplitude, stresses and operating life. For this purpose, existing horns were redesigned along with some new ones for evaluation of performance under longitudinal vibrations. The horn length, material and diameters at the transducer side and tool end were taken identical for all ultrasonic horn designs in order to have a fair comparison among performance parameters such as axial modal frequency, vibration amplitude, stresses, and factor of safety under same operating conditions. The length of horn was taken to be $114 \mathrm{~mm}$, whereas the horn diameters at transducer side and tool end were set equal to $41 \mathrm{~mm}$ and $33 \mathrm{~mm}$ respectively, based on the commercially available ultrasonic machining system. The horn material considered in this research was mild steel (S) with properties presented in Table 5 [12]. The operating frequency of ultrasonic generator was $20 \mathrm{kHz}$, whereas the amplitude of 
vibration at the transducer end was taken to be $5 \mu m[6,40]$. Another objective of this research was to design ultrasonic horn for soft and brittle composites to reduce stresses and enhance operating life without compromising the amplitude of vibration.

Table 5. Properties of ultrasonic horn material considered in this research.

\begin{tabular}{ccc}
\hline Properties & Symbol & Magnitude \\
\hline Density & $\rho$ & $7850 \mathrm{~kg} / \mathrm{m}^{3}$ \\
Poisson's Ratio & $v$ & 0.3 \\
Elastic Modulus & $E$ & $200 \mathrm{GPa}$ \\
Yield Strength & $\sigma_{Y}$ & $250 \mathrm{MPa}$ \\
Speed of Sound & $c$ & $5048 \mathrm{~m} / \mathrm{s}$ \\
Wave Length & $\lambda=c / f$ & $252 \mathrm{~mm}$ \\
Damping Factor & $\zeta$ & 0.013 \\
\hline
\end{tabular}

In this research, ultrasonic horn designs were divided into two unique categories, i.e., standard and hybrid horns. Standard ultrasonic horns include cylindrical, step, conical, catenoidal, Gaussian, exponential and Bezier horns. Hybrid ultrasonic horns were further divided into three categories; cylindrical hybrid horns, step hybrid horns and specialized complex hybrid horns. Cylindrical hybrid horns were developed by integrating cylindrical horn with one of standard ultrasonic horns such as conical, catenoidal, Gaussian, exponential and Bezier. The lengths of both cylindrical and standard profiles were taken to be $57 \mathrm{~mm}$. Performance of cylindrical hybrid horns was investigated by placing the cylindrical profile at the transducer (larger) end and then at the tool (smaller) end. The performance of cylindrical-catenoidal, catenoidal-cylindrical, cylindrical-Bezier, Bezier-cylindrical, conical-cylindrical, exponentialcylindrical, cylindrical-Gaussian and Gaussian-cylindrical horns have not been investigated in the past research.

Step hybrid designs were developed by incorporating one of standard horns such as conical, catenoidal, Gaussian, Bezier and exponential profiles at the location of abrupt change in cross section diameter. The length of larger cylinder at the transducer side and smaller cylinder at tool end were taken to be $57 \mathrm{~mm}$ and $27 \mathrm{~mm}$ respectively, whereas the lengths of conical, catenoidal, Gaussian, Bezier and exponential sections were set equal to $30 \mathrm{~mm}$. The influence of incorporating Gaussian and Bezier curves in ultrasonic step horn design have never been investigated in the past research in terms of vibration amplification, stresses and operating life.

The remaining ultrasonic horn designs from literature, which were either combinations of multiple step, cylindrical, conical, exponential profiles or involved some special geometric features were classified as specialized complex hybrid horns. The dimensions of such ultrasonic horns were found to be different in available literature depending on the size of the transducer and tool. However, the dimensions of specialized complex hybrid horns were adjusted and taken in similar proportions as considered in the past research.

Three dimensional CAD models of ultrasonic horns were developed for conducting modal and harmonic analyses. Solid Edge was used for three dimensional modeling of ultrasonic horns, whereas ANSYS was utilized for conducting finite element analysis. Modal analysis was done for computing modal frequencies and mode shapes, whereas harmonic analysis was carried out for computing amplitude of vibration, stresses, factor of safety and magnification factor numerically for $20 \mathrm{kHz}$ operating frequency of ultrasonic generator. Mesh independence analysis was carried out for accurate numerical results regarding resonant frequency, vibration amplitude and stresses [73, 74]. The amplitude of mechanical vibrations transmitted by the transducer was taken to be $5 \mu \mathrm{m}$ [6]. Therefore the harmonic displacement at the transducer end of the horn was set equal to $5 \mu \mathrm{m}$ in axial direction, and displacement amplitude at tool end was computed numerically. Since horn is a continuous system, therefore there are infinite mode shapes and hence modal frequencies associated with it. In ultrasonic machining systems, tool vibrates in the axial direction, thus only axial modal frequency is important in ultrasonic vibration assisted machining systems either stationary or rotary $[7,12,15,19,67]$. Simulation results for existing and newly designed ultrasonic horns were later compared to draw conclusion.

Research methodology, numerical computations and performance of ultrasonic horns were validated with research of Wang et al, Rani et al, Roy et al, Kumar et al, and Pang et al. by developing the geometric models of standard ultrasonic horns based on their dimensions and conducting finite element analysis for the determination of resonant frequency, vibration amplitude and stresses [7, 12, 15, 40, 57, 59]. Finally, those ultrasonic horns were preferred which provided greater vibration amplification, low stresses and higher operating life. Since amplitude of vibration required at the tool 
end is $6 \mu \mathrm{m}$ for the machining of Nomex honeycomb core material, therefore the results were found adequate for the design purpose [47].

\section{$4 \quad$ Results and discussions}

\subsection{Modal analysis}

Modal analysis was carried out for computing horn modal frequencies numerically [75]. Among infinite modal frequencies, axial modal frequency was given importance due to axial vibrations of ultrasonic machining system. The modal frequencies of step and $3^{\text {rd }}$ order Bezier horns were observed to be much higher as compared to other standard ultrasonic horns such as conical, catenoidal, $2^{\text {nd }}$ order Bezier and Gaussian as presented in Fig. 2a. Horn diameters at transducer and tool ends, material and length were identical for all standard ultrasonic horns, but modal frequencies were observed to be different because of dissimilarity among horn geometries, which influenced mass $m$ and thus axial modal frequency [70]. Axial modal frequencies of ultrasonic horns based on step design and $3^{\text {rd }}$ order Bezier curve were observed to be higher due to larger stiffness and lesser mass respectively [76]. Gaussian horn was observed to have higher stiffness but larger mass as well, resulting in moderate axial frequency. The comparison of mass and axial stiffness for standard ultrasonic horns is presented in Fig. $2 b$ \& $2 c$ respectively.
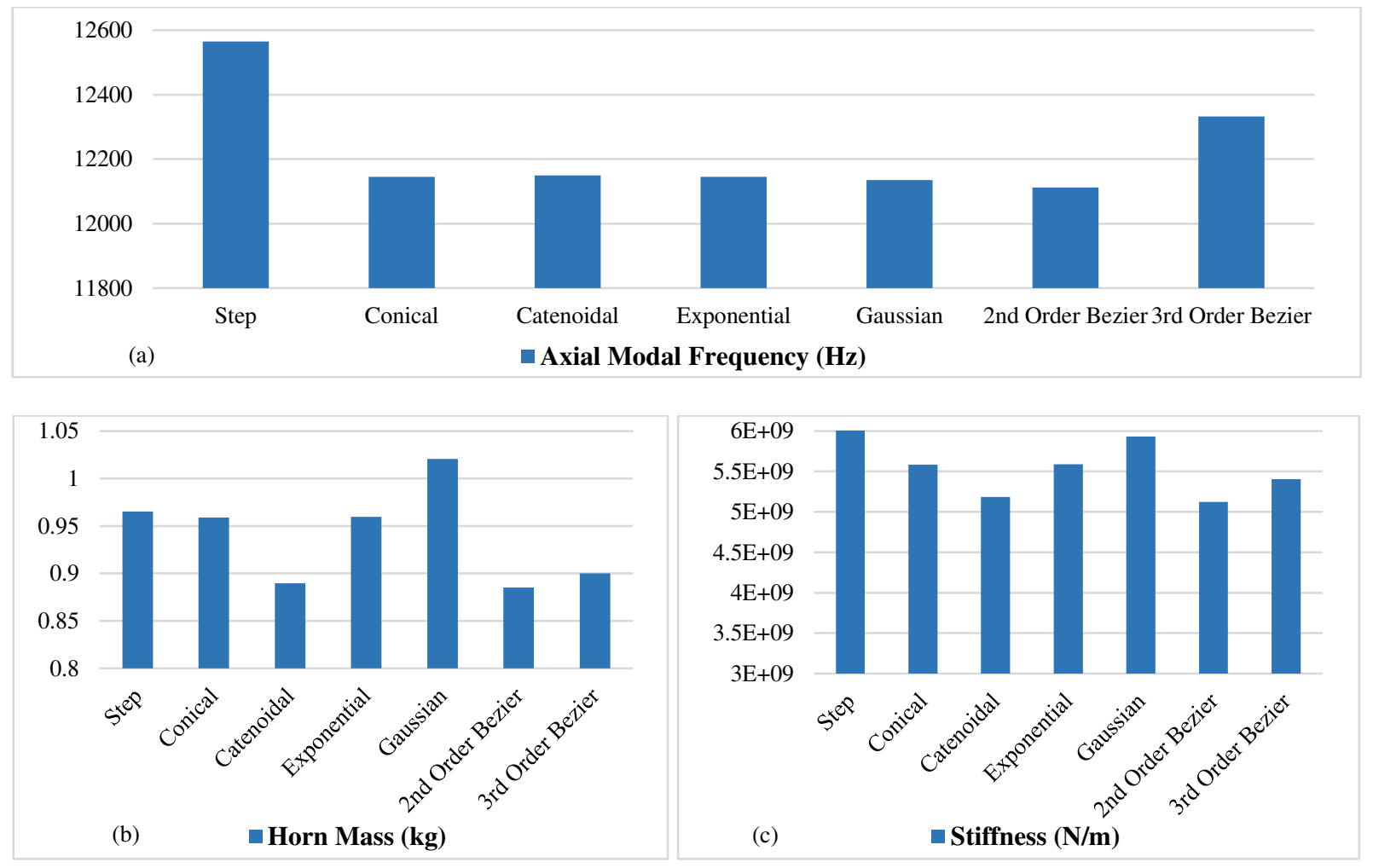

Fig. 2. Comparison of standard ultrasonic horn designs in terms of a) axial modal frequency, b) mass, c) axial stiffness

When standard ultrasonic horn profiles were integrated with the cylindrical portion and modal analysis was carried out, the axial modal frequency was observed to be different as compared to their standard counterparts as presented in Fig. 3a. Generally, the axial modal frequency was observed to become higher as compared to standard profiles when the cylindrical portion was placed near the transducer end, while reduce when the cylindrical portion is incorporated at the tool end of horn. The modal frequency of cylindrical-catenoidal, Gaussian- cylindrical and cylindrical-Bezier horns were observed to be higher among family of cylindrical horns. The modal frequency of conical and exponential horn was observed to reduce, while increase for that for catenoidal, Gaussian and Bezier horns when incorporated with cylindrical profile at the transducer side. Gaussian-cylindrical horn was observed to have higher axial frequency due to higher axial stiffness and lesser mass. The mass and axial stiffness for those cylindrical 
hybrid horns were observed to be higher for which cylindrical portion was used at the transducer side as shown in Fig. $3 \mathrm{~b}$ and $3 \mathrm{c}$.
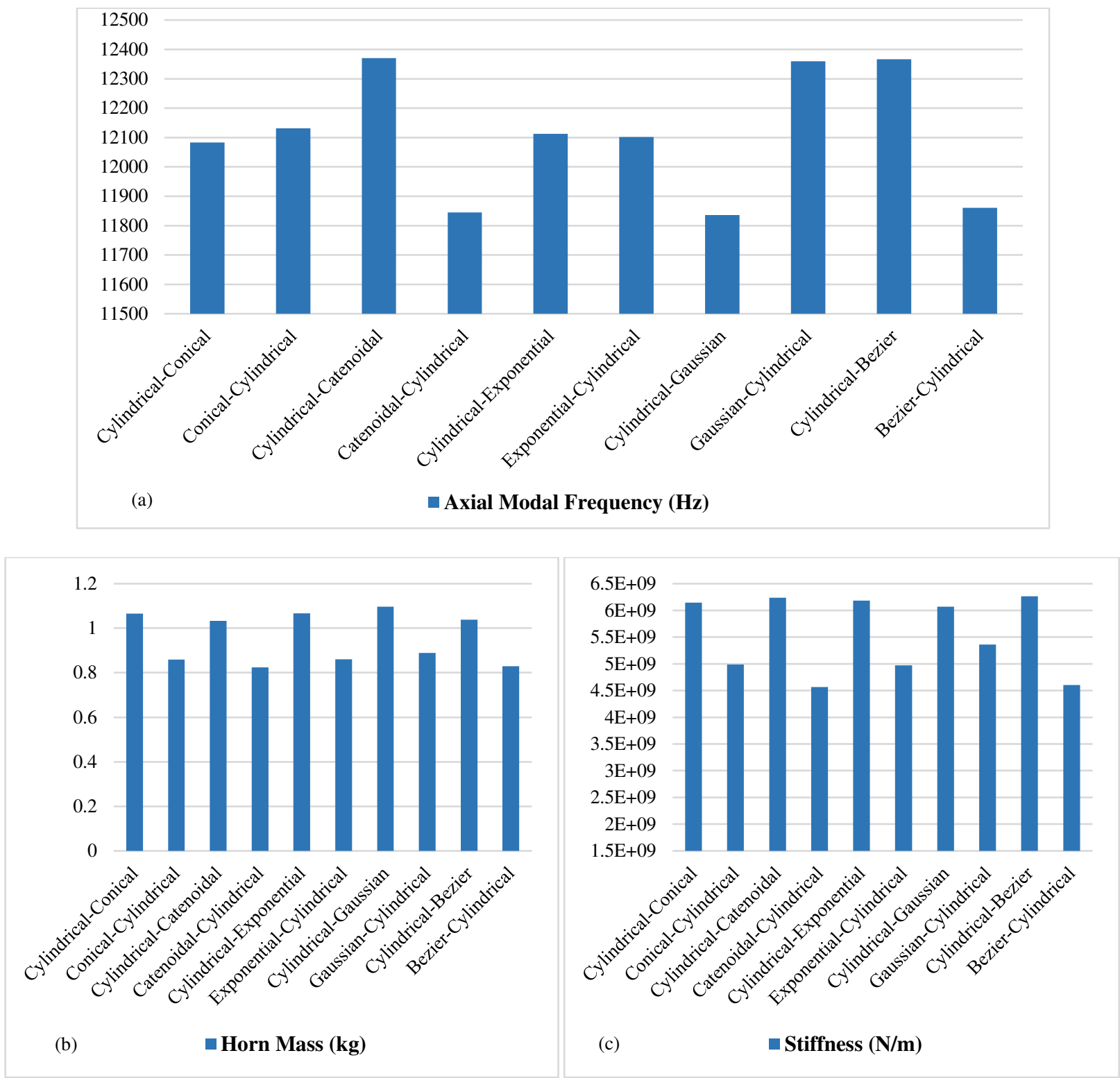

Fig. 3. Comparison of cylindrical hybrid ultrasonic horn designs in terms of a) axial modal frequency, b) mass, c) axial stiffness

When the conical and catenoidal profiles were incorporated in the step design at the location of changing cross sectional area, the axial modal frequency was observed to increase as compared to standard step horn. The axial modal frequency was observed to decrease when exponential, Gaussian and step profiles were used at step location. Largest axial modal frequency was achieved when conical profile is incorporated at the location of changing cross section as presented in Fig. 4a. However, when the conical profile was incorporated after the step horn at the tool end, significant drop in axial modal frequency was observed due to reduction in axial stiffness. Small variation in horn mass and axial stiffness was observed when standard curves and line were incorporated in step horn at the location of abrupt change in cross sectional area as presented in Fig. $4 \mathrm{~b}$ and $4 \mathrm{c}$. 

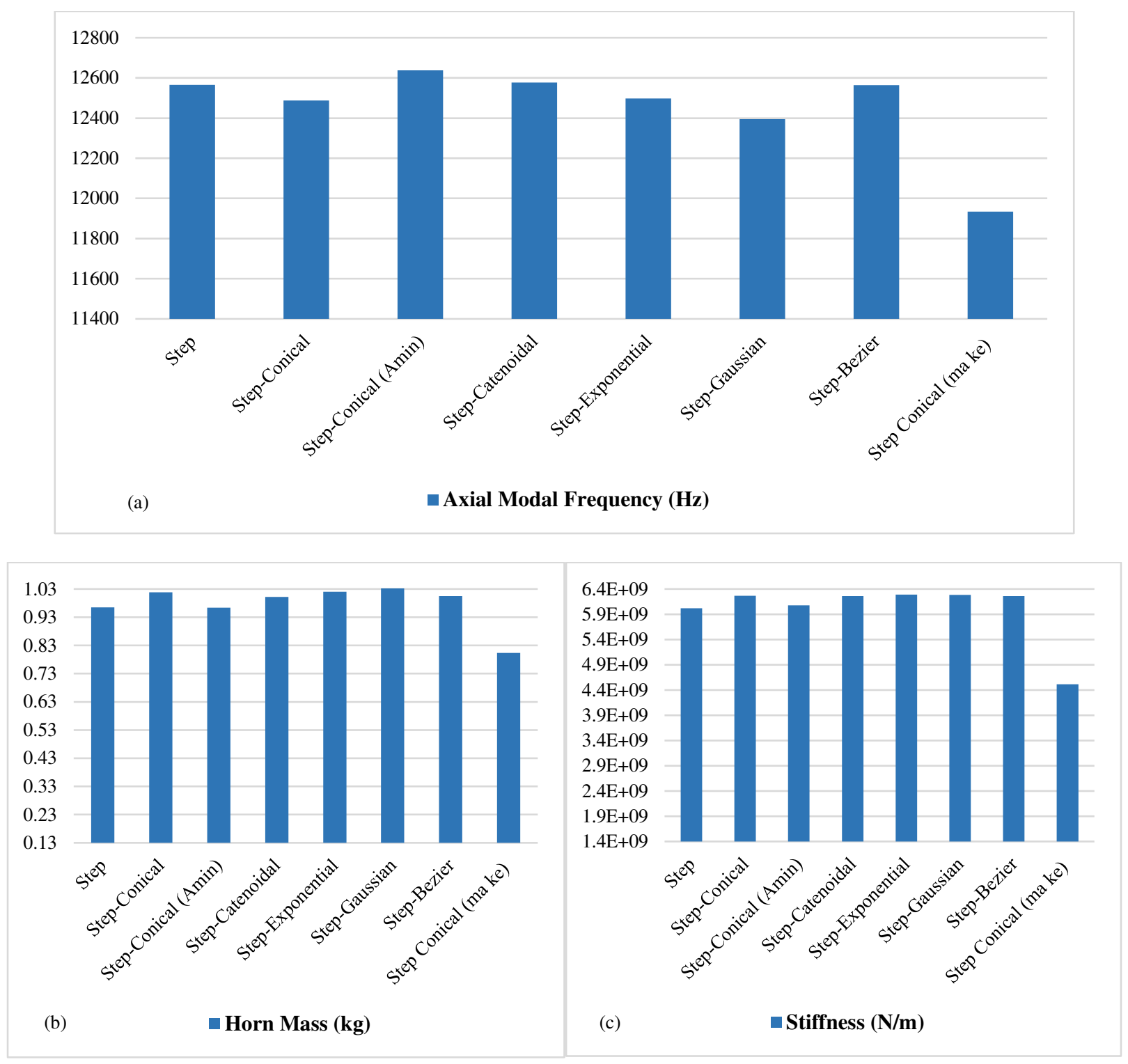

Fig. 4. Comparison of step hybrid ultrasonic horn designs in terms of a) axial modal frequency, b) mass, c) axial stiffness

The comparison of axial modal frequency for complex hybrid ultrasonic horns, presented in Fig. 5a, indicated that some specialized hybrid horns, such as barrel, step-double conical, cylindrical- double conical and hollow exponential, achieved axial modal frequency greater than that of step horn. The axial modal frequency of barbell horn, spool horn and cylindrical horn with cuboid hole were observed to be very low as compared to all ultrasonic horns considered in this article, as a result of reduction in axial stiffness primarily due to involvement of smallest cross sectional area [70, 76]. The comparison of mass and axial stiffness for complex hybrid ultrasonic horn designs are presented in Fig. 5b and $5 \mathrm{c}$. Barrel horn has highest stiffness, therefore its axial modal frequency and vibration amplification was observed to be highest among all ultrasonic horn designs considered in this research. 

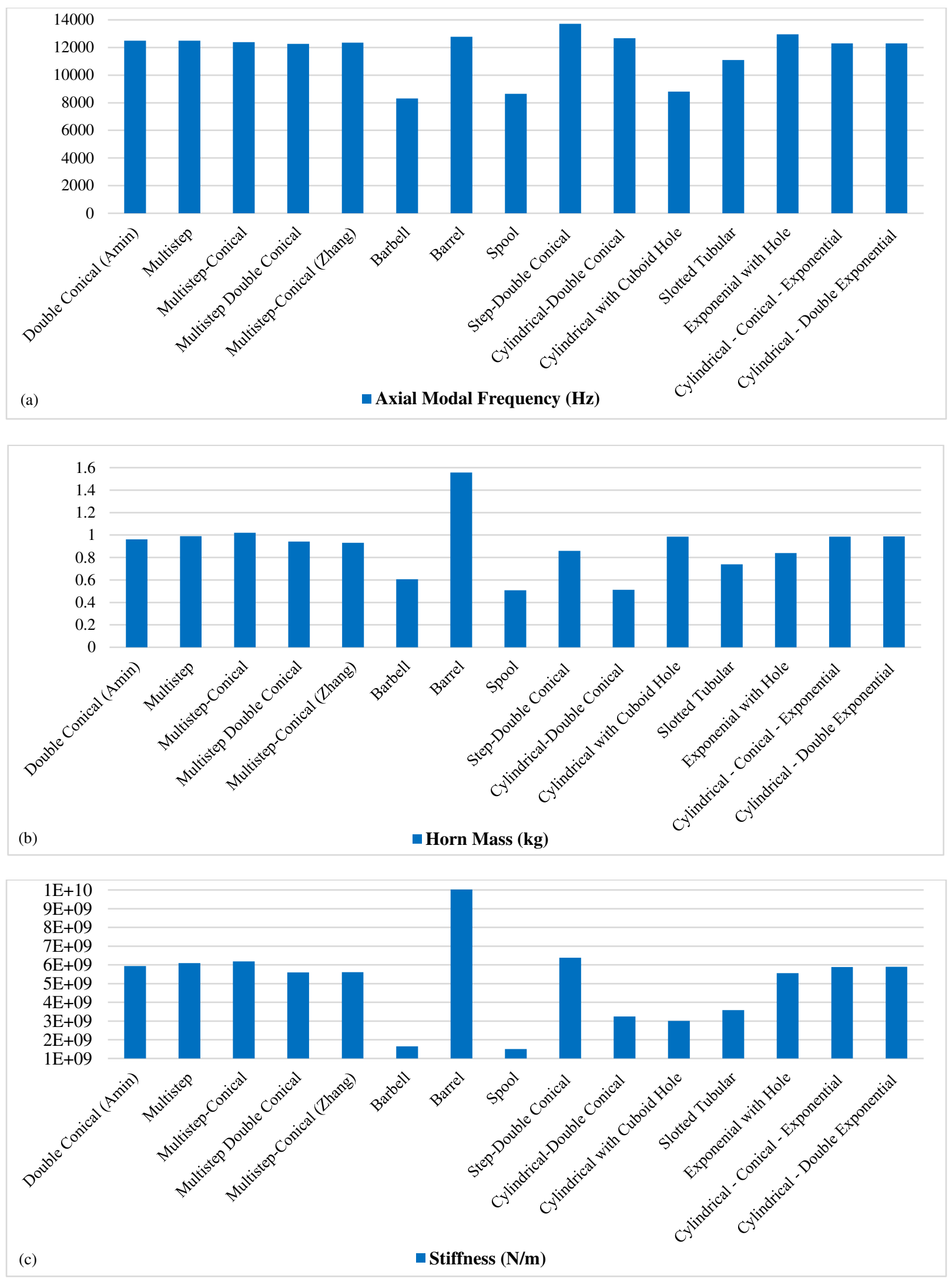

Fig. 5. Comparison of specialized complex hybrid ultrasonic horn designs in terms of a) axial modal frequency, b) mass, c) axial stiffness 


\subsection{Harmonic analysis}

Harmonic analysis of ultrasonic horns was performed after modal analysis, for computing numerically the vibration amplitude, magnification factor and stresses [75]. Since the horn material for all ultrasonic horns considered in this research is steel which is ductile in nature at operating conditions, therefore only Von Mises stresses were analyzed [77]. The contour plots for axial displacement for partticular ultrasonic horns with higher vibration amplification are presented in Fig. 6. The maximum displacement achieved by ultrasonic horns was observed at tool end justifying the effectiveness of horn designs and finite element methods considered in this research. Among standard ultrasonic horn profiles, the amplitude of vibration for step design was observed to be greatest because its axial modal frequency was closer to excitation frequency of generator. The vibration amplification achieved through stepped horn was calculated analytically by using $M_{\text {step }}=(D / d)^{2}$ [13], which was found equal to 1.55 for the current horn dimensions, showing the effectiveness and reliability of the finite element methods. The vibration amplification achieved by $3^{\text {rd }}$ order Bezier horn was observed larger as compared to conical, catenoidal, exponential, Gaussian and $2^{\text {nd }}$ order Bezier horns, however $11.74 \%$ lesser as compared to that of step horn. This indicated a room to further improve the vibration amplification to get closer to step design. Generally, the amplitude of vibration is maximum at resonance i.e., when the excitation frequency of generator transmitted through transducer matches with horn's axial modal frequency $\left(\omega=\omega_{n}\right)$ [76]. The comparison of vibration amplitude achieved through standard ultrasonic horn designs are presented in Fig. 7.

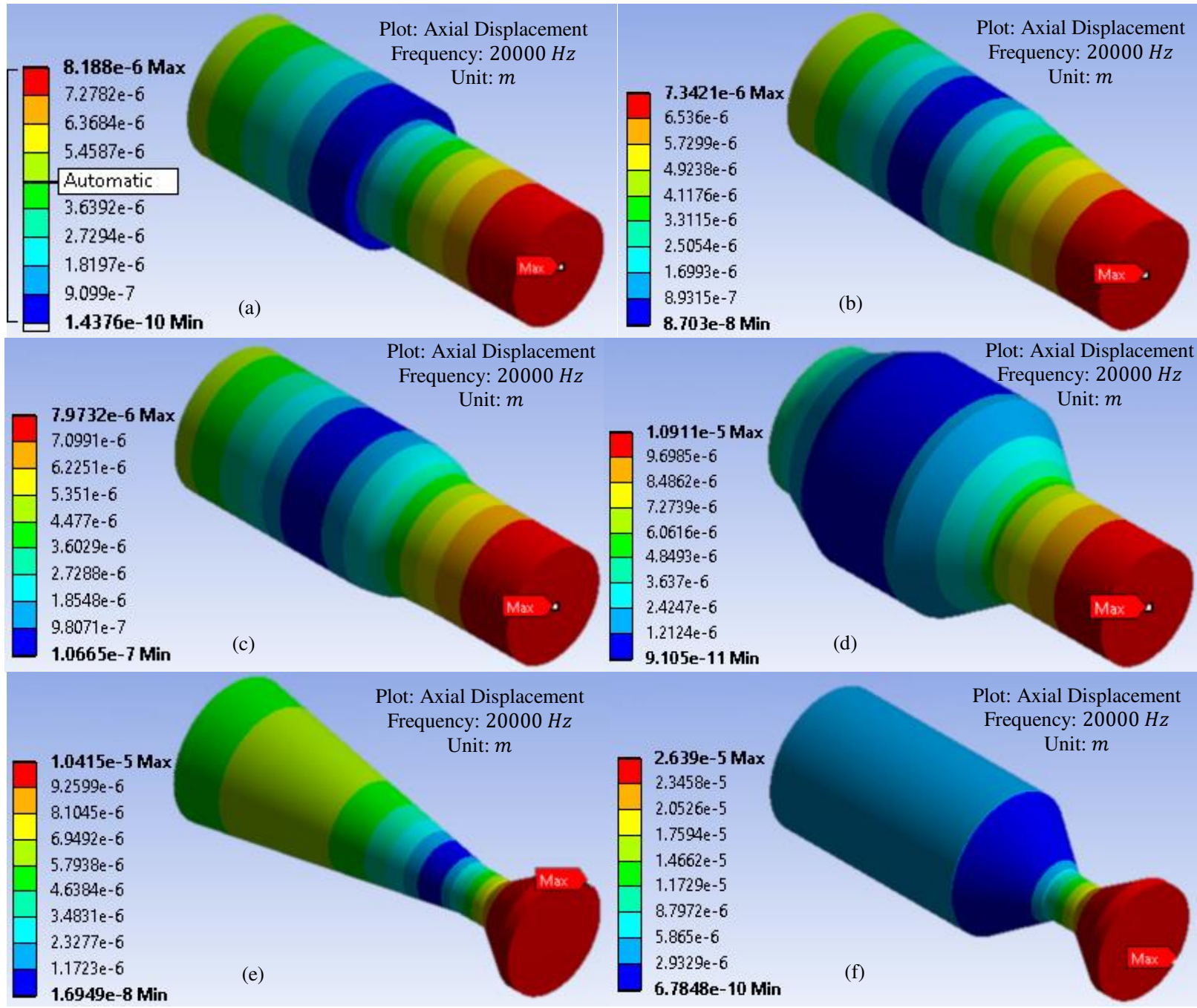

Fig. 6. Contour plots of axial displacement indicating vibration amplitudes for a) step horn, b) step-catenoidal horn, c) step-Bezier horn, d) barrel horn, e) cylindrical-double conical horn, e) step-double conical horn 


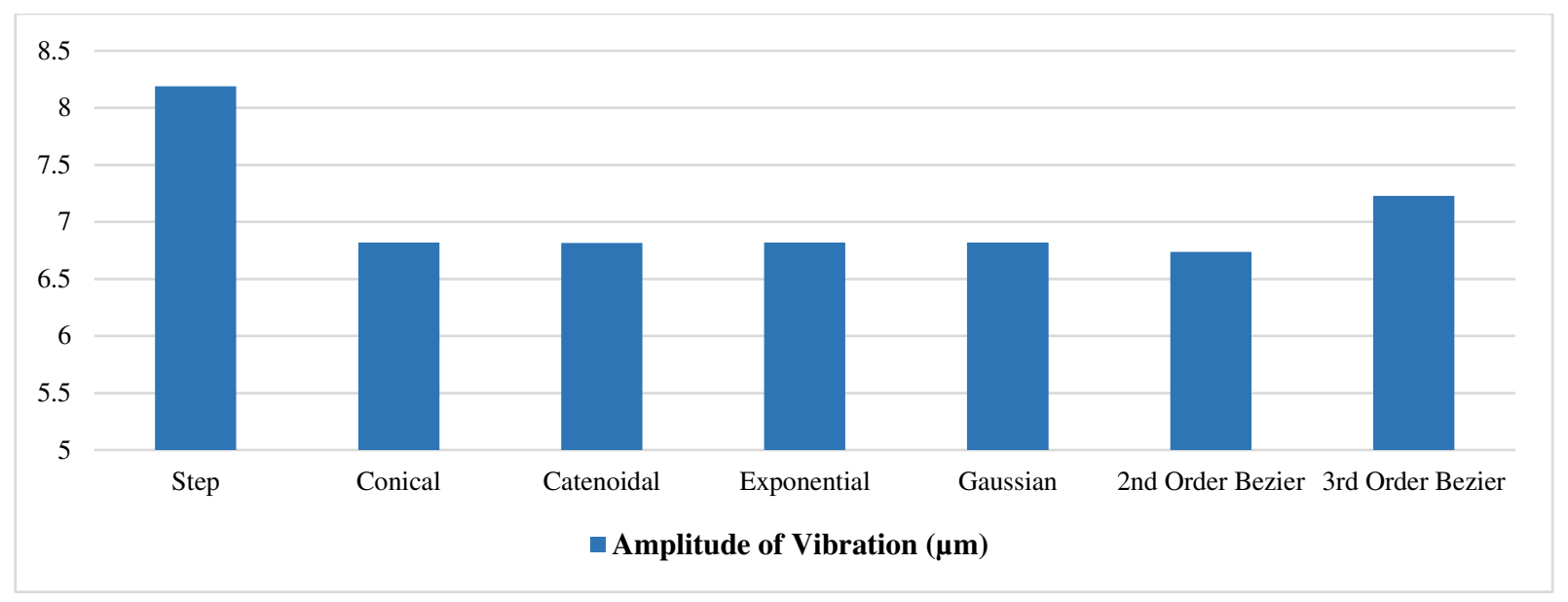

Fig. 7. Comparison of amplitude of vibration for standard ultrasonic horn designs

For cylindrical family of ultrasonic horns, higher amplitude of vibration, thus magnification factor was achieved when cylindrical portion was placed at transducer side and connected to catenoidal, exponential and Bezier profiles respectively. However, larger vibration amplification was achieved in case of Gaussian-cylindrical horn, when cylindrical portion was placed at the tool end. The vibration amplification achieved by cylindrical-catenoidal, Gaussian-cylindrical and cylindrical-Bezier horns was observed to be $10.38 \%, 10.87 \%$ and $10.5 \%$ lesser as compared to step horn respectively, while approximately $1 \%$ higher as compared to that of $3^{\text {rd }}$ order Bezier horn. The amplitude of vibration and magnification factor was observed higher for those ultrasonic horns whose axial modal frequency was closer to exciting frequency of the generator. The comparison of vibration amplitude achieved through cylindrical hybrid ultrasonic horn designs are presented in Fig. 8.

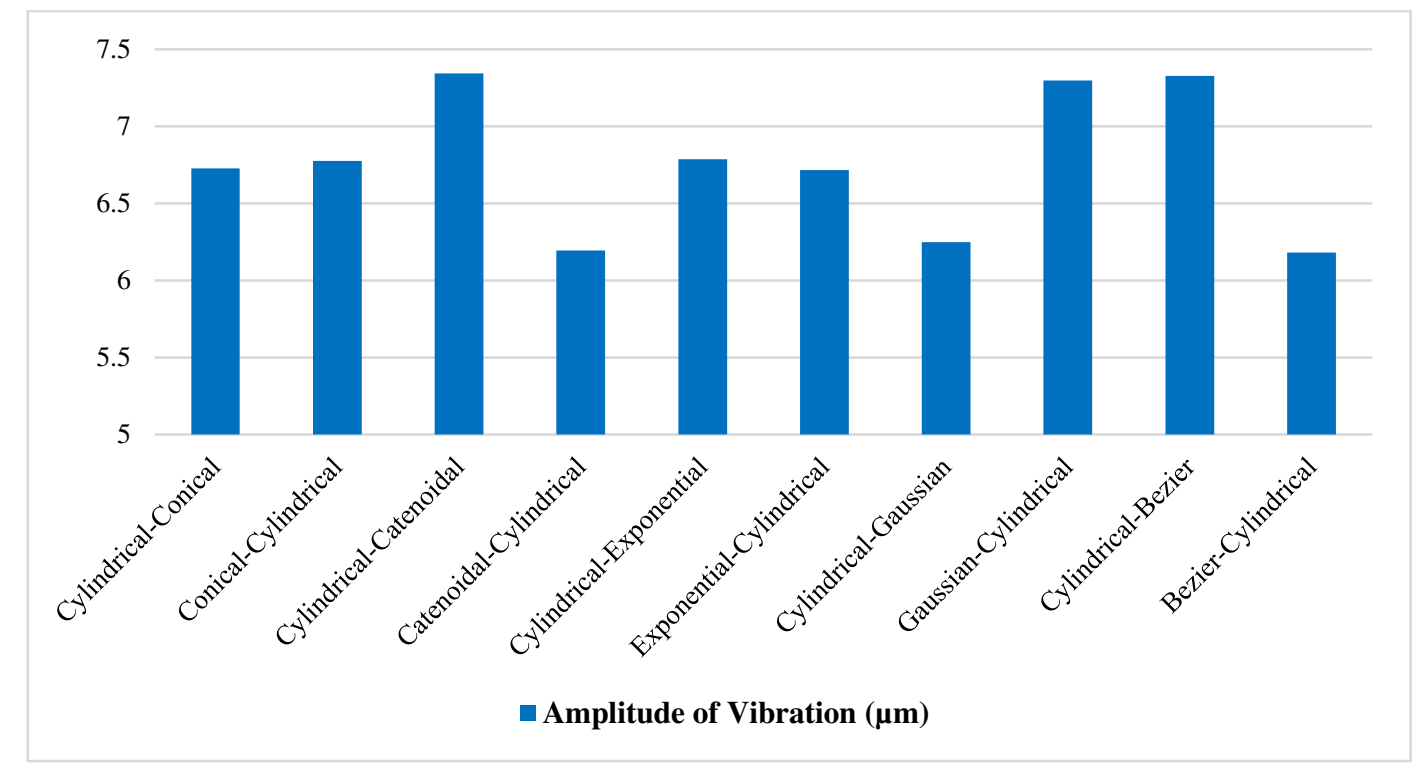

Fig. 8. Comparison of amplitude of vibration for hybrid cylindrical ultrasonic horn designs

The amplitude of vibration and magnification factor were observed to drop when standard ultrasonic horn profiles were incorporated in step design at the location of abrupt change in diameter. The vibration amplification achieved by step-conical, step-catenoidal and step Bezier horns were observed to be very close to that of step horn by $0.61 \%, 2.81$ $\%$ and $2.69 \%$, respectively. However, when the conical section is attached at the end of step horn, instead at the location of abrupt change in cross sectional area, the vibration amplification was observed to drop significantly. The comparison of vibration amplitude achieved through step hybrid ultrasonic horn designs are presented in Fig. 9. 


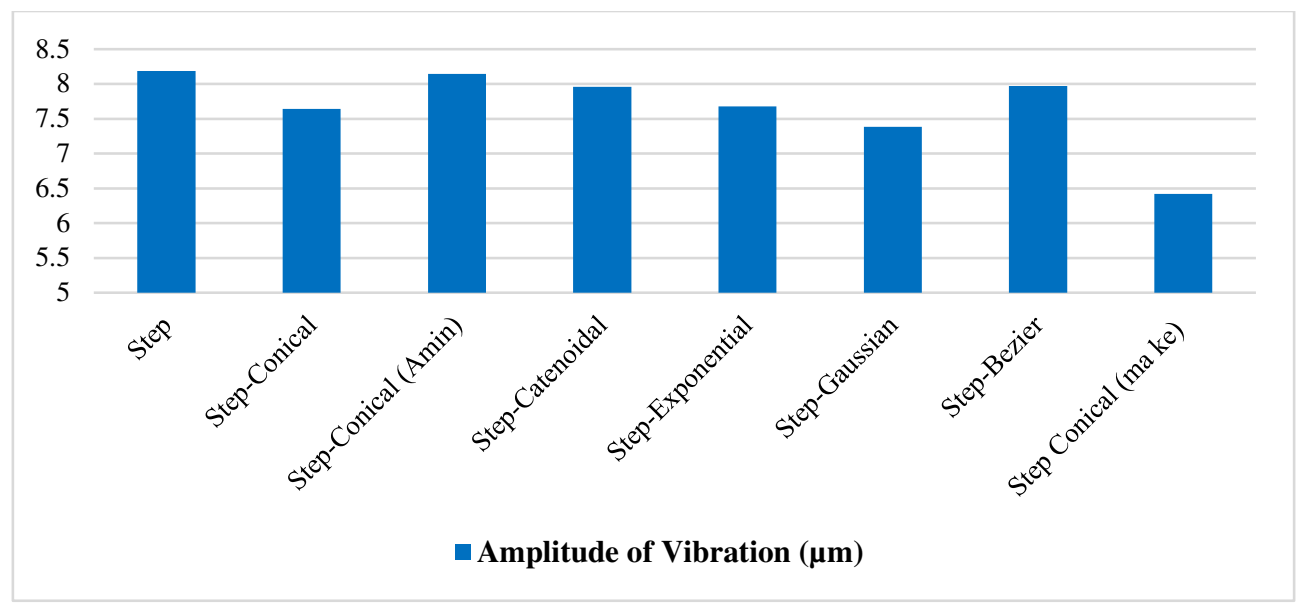

Fig. 9. Comparison of amplitude of vibration for hybrid step type ultrasonic horn designs

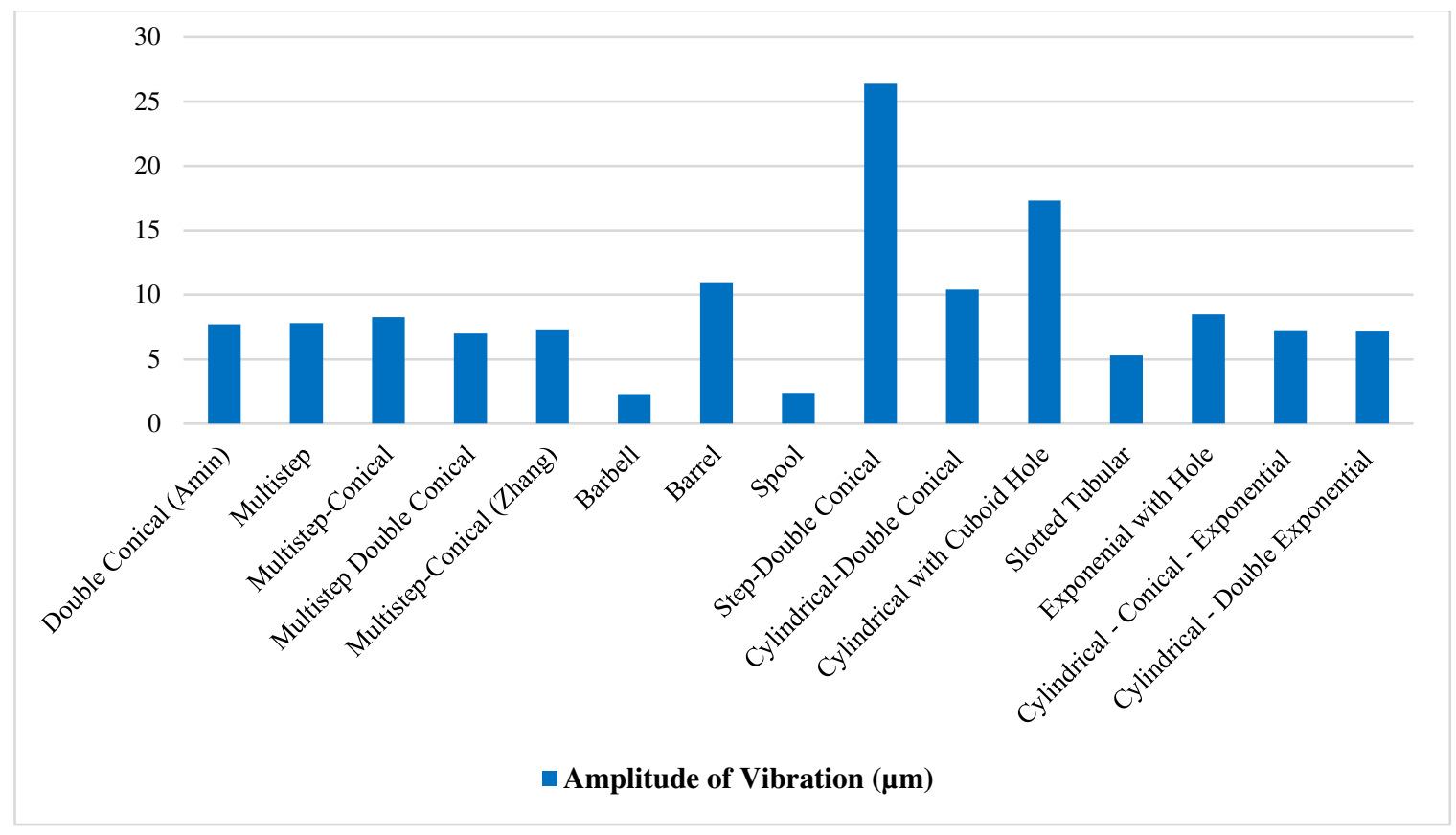

Fig. 10. Comparison of amplitude of vibration for specialized hybrid ultrasonic horn designs

The comparison of vibration amplitude achieved through complex hybrid ultrasonic horn designs are presented in Fig. 10. The amplitude of vibration and magnification factor were observed to be greater in case of barrel horn, step-double conical horn, cylindrical-double conical horn, hollow exponential horn, multistep-conical horn and cylindrical horn with cuboid hole as compared to that of step horn. The vibration amplification was observed to be greater in those ultrasonic horns, whose axial modal frequency was closer to the excitation / operating frequency of ultrasonic system. However, the axial modal frequency of cylindrical horn with cuboid hole was extremely low and $29.95 \%$ lesser but vibration amplification was observed to be $111.6 \%$ greater as compared to that of step horn. For proper working of ultrasonic system, efficient energy transmission and vibration amplification, first axial mode must be closer to excitation frequency. For cylindrical horn with cuboid hole, the first axial mode was found to be far away from the excitation frequency of the generator, whereas the second axial mode was observed at $13715 \mathrm{~Hz}$ with vibration amplitude of $17.33 \mu \mathrm{m}$ as presented in Fig. 11. In this mode, only tool end was observed to vibrate with relatively higher amplitude as compared to other portions of ultrasonic horn, which was not desired for smooth machining operations and other ultrasonic applications. Therefore, performance of cylindrical horn with cuboid hole could not be considered superior in terms of vibration amplification. 
On the other side, the amplitude of vibration achieved by hollow exponential, barrel, step-double conical, multistepconical and cylindrical-double conical ultrasonic horns were observed to be 3,66 \%,33.21\%,222.22\%,1\% and $27.23 \%$ higher respectively as compared to that of step horn. The magnification factor for theses horns were observed to be greater as compared to step design because of higher axial modal frequencies and frequency ratios relatively closer to 1 . Furthermore, the involvement of shorter horn diameter at specific locations than the diameter at tool end in those horn designs also caused higher vibration amplification.

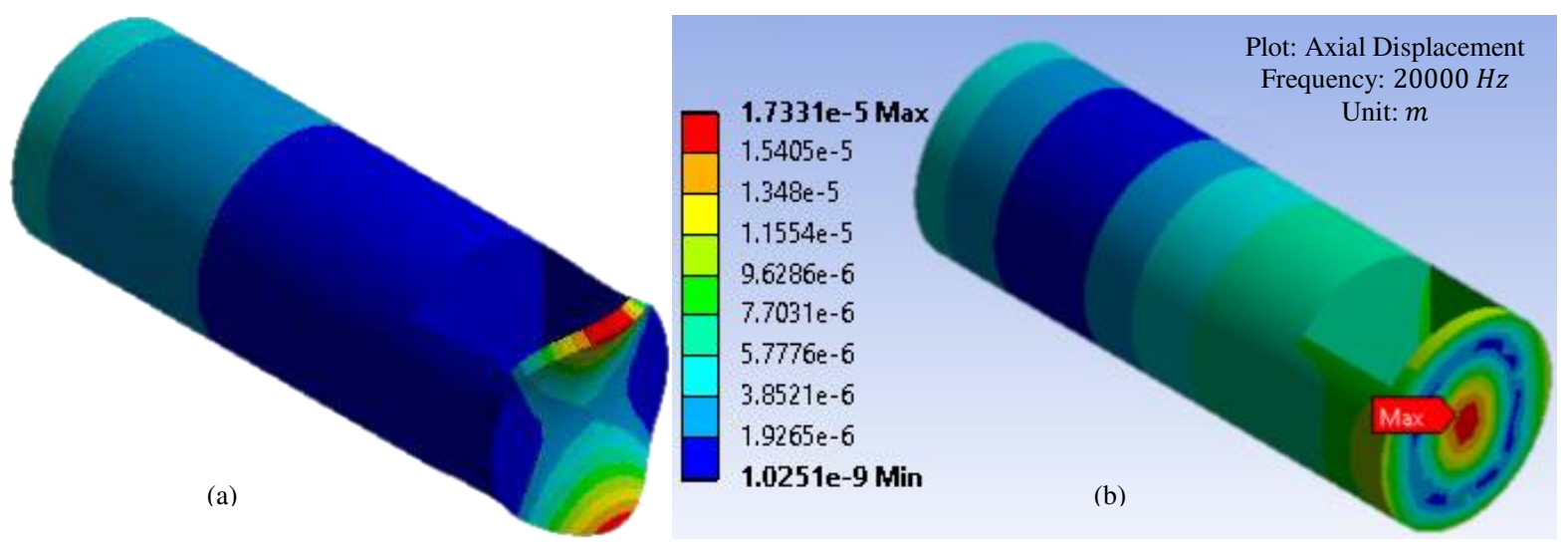

Fig. 11. Countour polts for cylindrical horn with cuboid hole illustrating a) second axial mode of vibration, b) axial displacement and amplitude of vibration

\subsection{Stress analysis}

The aim of the current research was to evaluate existing state of the art ultrasonic horn designs in terms of stresses, vibration amplification and operating life. The idea was to explore ultrasonic horn designs with higher vibration amplification at tool end, minimum stresses and more operating life. For this purpose, existing horn shapes were used and some new horns were designed by integrating standard horn profiles based on the dimensions of commercially available ultrasonic machining system. For the assessment of stresses and operating life, Von Mises stresses produced in ultrasonic horns with fixed length, and transducer side and tool end diameters, were then computed numerically when vibration frequency of $20 \mathrm{kHz}$ was applied at the transducer end of horn [75]. The contour plots of stress for particular ultrasonic horn designs with larger vibration amplification are presented in Fig. 12. The stresses developed in ultrasonic horns were observed to be significantly greater in axial direction as compared to radial, tangential and other directions due to ultrasonic vibrations. Also, the ultrasonic horn material considered in this research was ductile in nature, therefore axial stresses would not predict failure. For this purpose, Von Mises stresses were computed by using either principal stresses, or axial, radial and tangential stresses [12, 25, 57, 72]. Furthermore, the stresses are dynamic in nature and depends on horn geometry, frequency ratio (ratio of excitation frequency to modal frequency), vibration amplitude, cross sectional area, mass, acceleration and transducer output, therefore the stresses produced in various horn designs were observed to be different. The comparison of maximum Von Mises stresses developed in standard ultrasonic horn designs is presented in Fig. 13. The stresses were observed to be greatest in step horn design due to abrupt change in cross sectional area resulting in high stress concentrations. The stresses were found to be significantly reduced in conical, Bezier, catenoidal, Gaussian and exponential horn designs due to smooth variation in cross sectional area. The stresses in Gaussian horn were observed to be least with magnitude $44.17 \%$ lesser as compared to that of step horn. The stresses in $3^{\text {rd }}$ order Bezier horn were observed to be $37.67 \%$ lesser as compared to step horn, whereas $11.64 \%$ and $8.95 \%$ greater as compared to Gaussian and $2^{\text {nd }}$ order Bezier horns respectively. This indicated that $3^{\text {rd }}$ order Bezier horns would be suitable for high vibration amplification, low stress concentrations and more operating life in ultrasonic applications.

The stresses were observed to reduce when standard horn profiles were integrated with the cylindrical profile as indicated in Fig. 14. Generally, the stresses were found to be lesser in cylindrical family of horns, when cylindrical profile was incorporated at the transducer side due to smooth transition of straight profile to curved profile. However, the stress concentrations were observed to be slightly larger when cylindrical profile was used at the tool end, possibly due to mismatch of slops at the transition section. In cylindrical horn family, least stresses were observed in cylindrical-Gaussian horn with a drop of $46.88 \%$ as compared to that of step horn. 


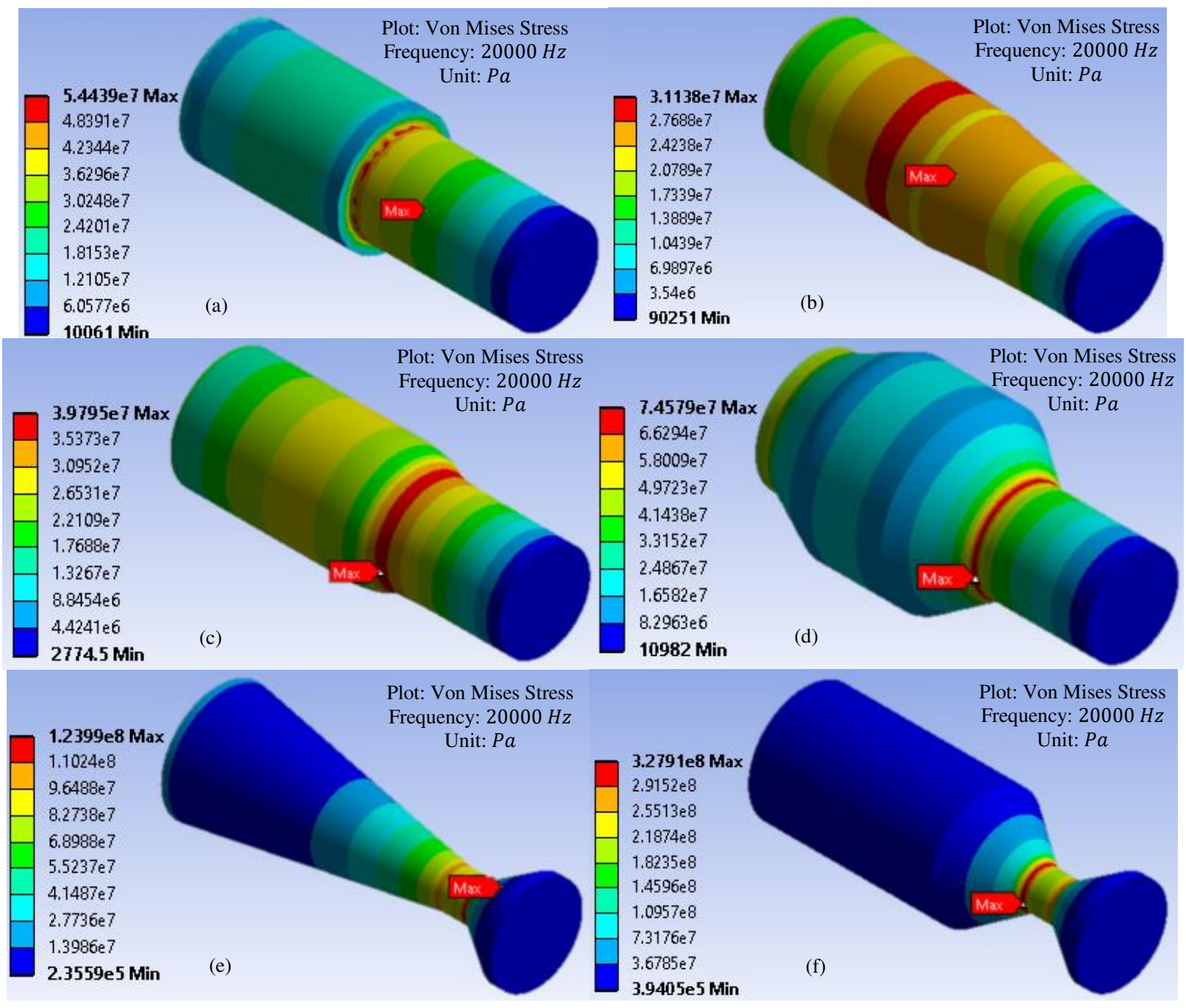

Fig. 12. Contour plots of Von Mises Stress for a) step horn, b) step-catenoidal horn, c) step-Bezier horn, d) barrel horn, e) cylindrical-double conical horn, e) step-double conical horn

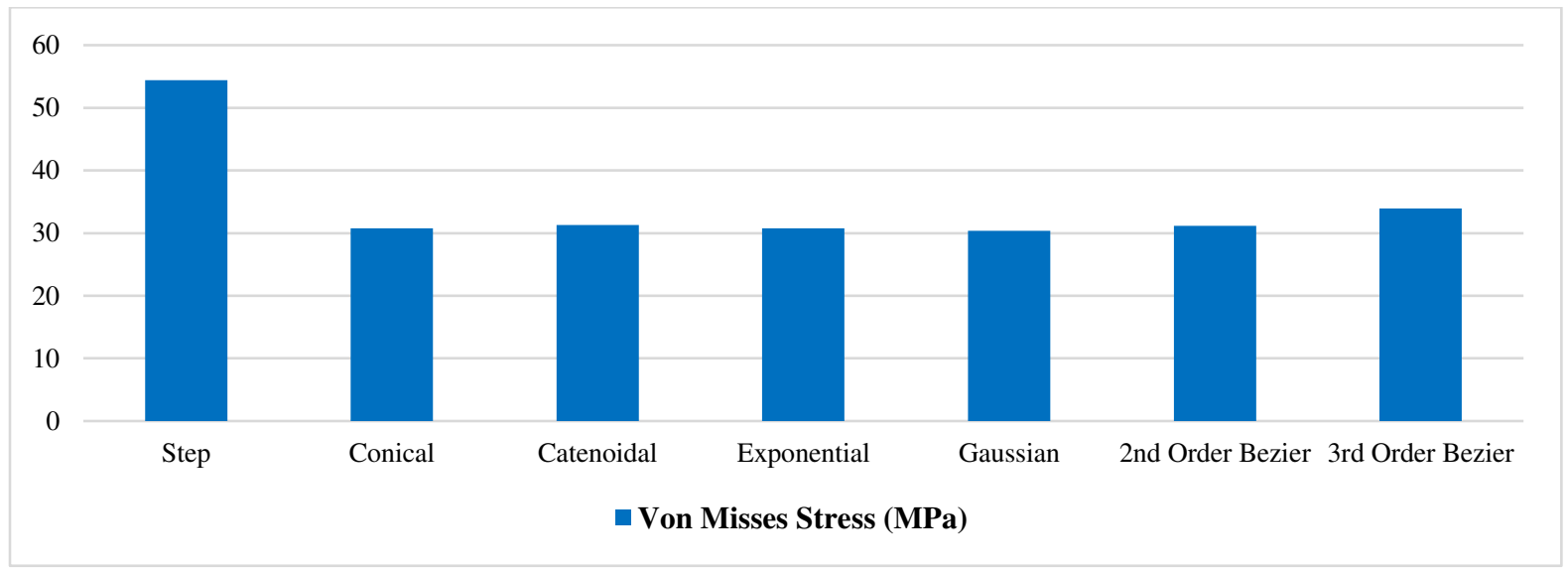

Fig. 13. Comparison of Von Mises stresses for standard ultrasonic horn designs 


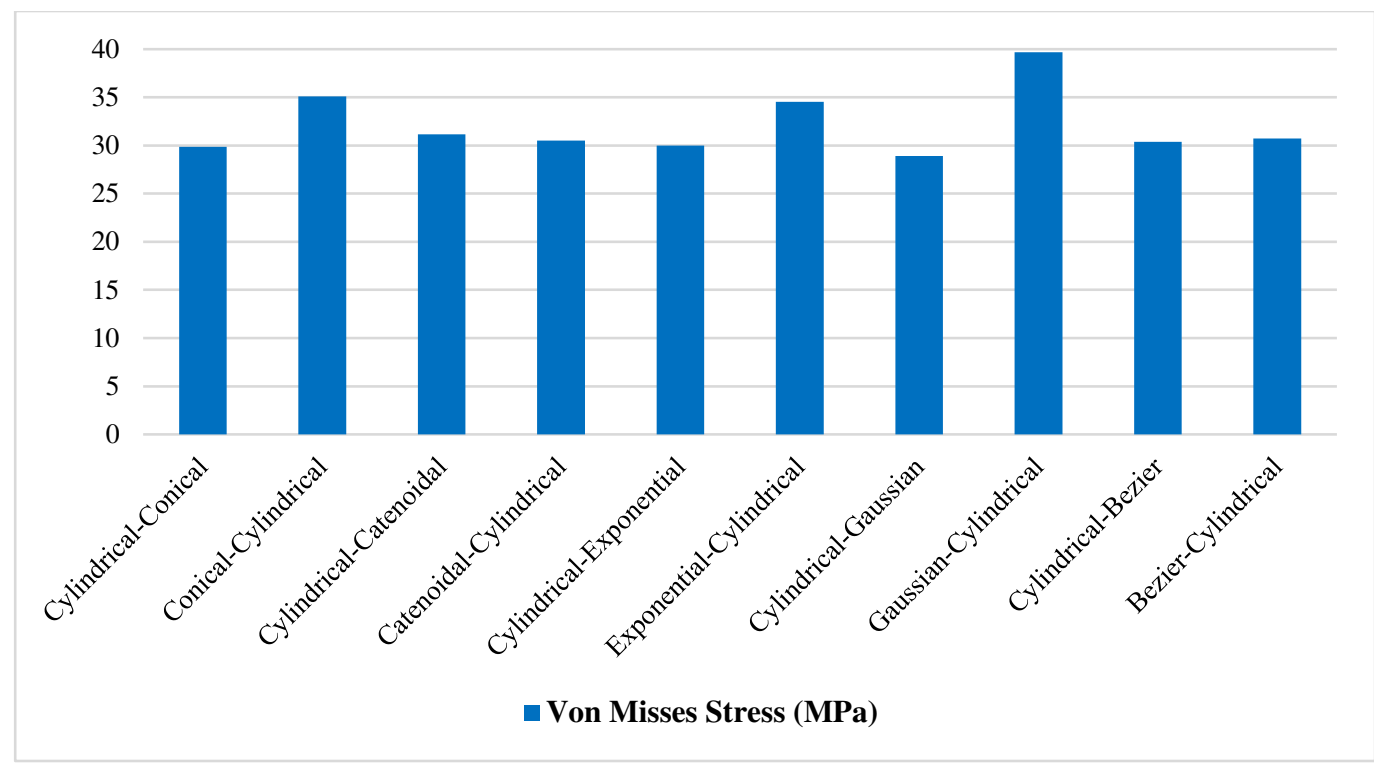

Fig. 14. Comparison of Von Mises stresses for cylindrical hybrid ultrasonic horn designs

When the standard horn geometries were incorporated in the step horn at the location of abrupt change in cross sectional area, the stresses were observed to be reduced significantly, as expected. Least stresses were produced in step-Gaussian horn with reduction of $44.25 \%$ as compared to that of step horn. The stresses were observed to decrease by $26.36 \%, 39.73 \%, 41.99 \%, 26.89 \%$ and $20.52 \%$ in step-conical, step, catenoidal, step-exponential, step-Bezier and multistep horn designs respectively as compared to that of step horn. The comparison Von Mises stress produced in step hybrid ultrasonic horn designs is presented in Fig. 15.

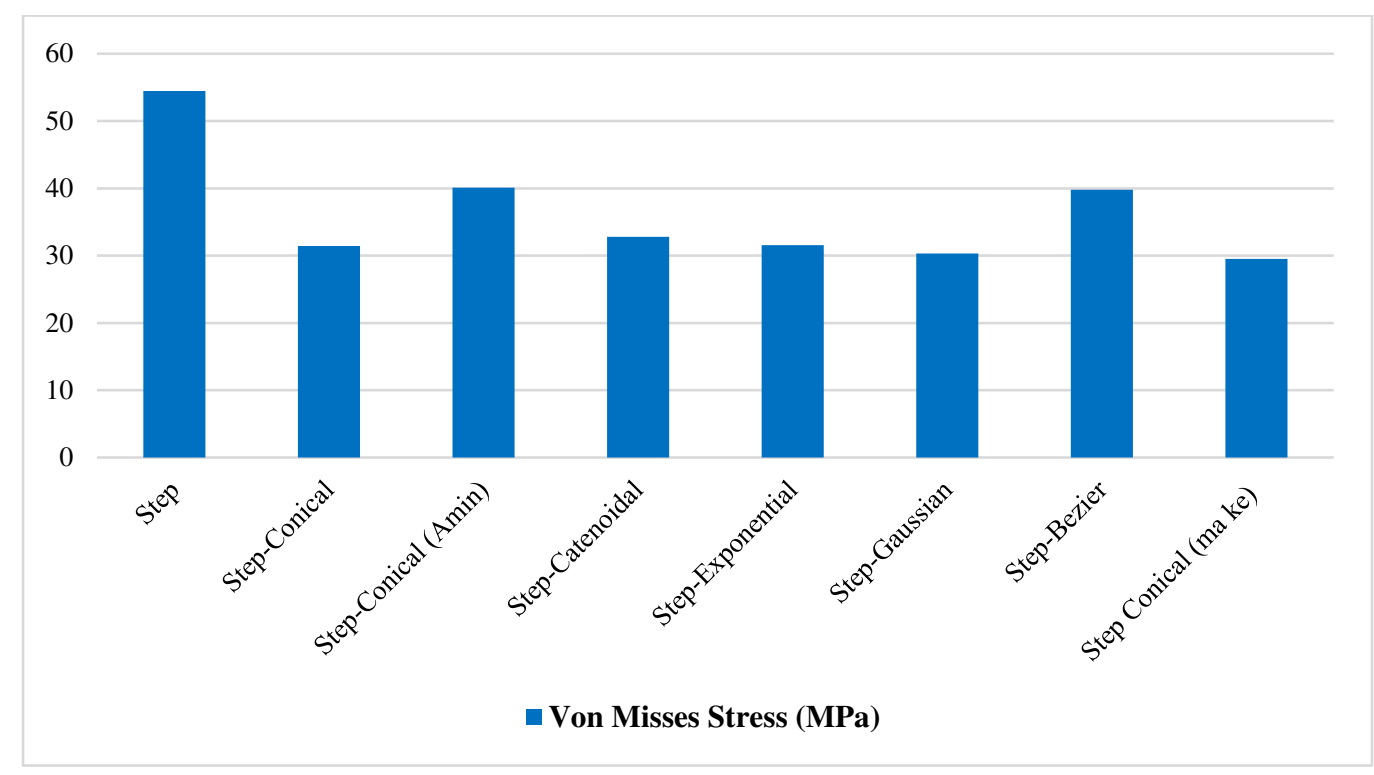

Fig. 15. Comparison of Von Mises stresses for step hybrid ultrasonic horn designs

The Von Mises stresses for all ultrasonic horns were observed to be in acceptable range except step-double conical design for which the stresses were found to be $502 \%$ and $866 \%$ higher as compared to step and Bezier horn designs respectively, as presented in Fig. 16. Similarly, the stresses were observed to be $423 \%$ and $265 \%$ higher in cylindricaldouble conical horn as compared to that of step and Bezier horns respectively. The stresses were observed to be very high due to the involvement of very short diameter near the tool end, that also resulted in higher modal frequency and vibration amplification. Stresses were observed to be $24.79 \%, 20.52 \%, 6.09 \%, 29.07 \%, 1.69 \%, 41.88 \%, 17.06 \%$ 
and $41.98 \%$ lesser in double conical, multistep, spool, slotted tubular, hollow exponential, cylindrical-conicalexponential, multistep-conical and cylindrical-double exponential horns respectively, as compared to step horn. However, the stresses were found to be larger in double conical, multistep, multistep-conical, spool, slotted tubular, hollow exponential designs by $20.66 \%, 27.52 \%, 33.07 \%, 50.68 \%, 13.80 \%$ and $57.73 \%$ respectively, and lesser in cylindrical-conical-exponential and cylindrical-double exponential horns by $6.75 \%$ and $6.92 \%$ as compared to Bezier horn. The stresses in Barbell, cylindrical with cuboid hole and Barrel horn designs were observed to be higher by $15.83 \%, 82.35 \%$ and $37.00 \%$ as compared to step design and $119.80 \%, 192.56 \%$ and $119.80 \%$ as compared to that of Bezier horn respectively. The barbell and spool horn designs were observed to be non-suitable due to extremely low vibration amplification and relatively less operating life.

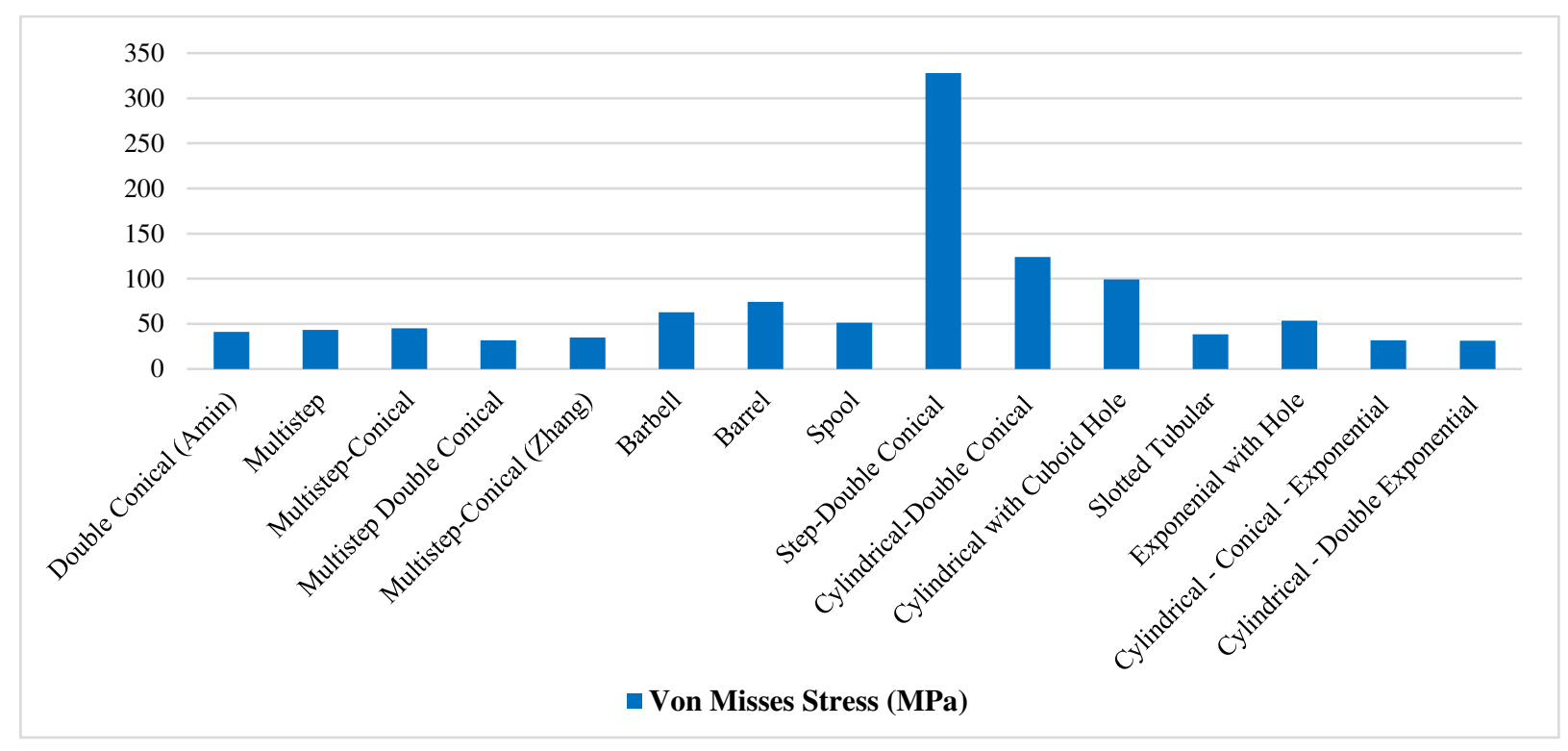

Fig. 16. Comparison of Von Mises stresses for specialized complex hybrid ultrasonic horn designs

For larger factor of safety, thus operating life, the stresses developed in the ultrasonic horn must be as lesser as possible than the yield strength of the material. For the determination of most suitable ultrasonic horn for the machining of soft and brittle composites such as Nomex honeycomb material, the factor of safety for each horn geometry was computed by using the maximum Von Mises stress and yield strength of the material. However, for the final decision regarding the best ultrasonic horn profiles, the factor of safety of those ultrasonic horns were presented in Fig. 17, which achieved higher vibration amplification, as well, at the tool end. This plot indicates the comparison of ultrasonic horn profiles considered in this research with higher vibration amplification and factor of safety. The actual factor of safety would be lesser than the computed values owning to possible reduction in yield strength due to certain factors depending on operating conditions These factors may include reliability factor, temperature factor, size factor, stress concentration and some other miscellaneous factors [78].

Among ultrasonic horns with higher vibration amplification comparable with step design, the highest factor of safety was observed for cylindrical-Bezier horn. The amplitude of vibration achieved by cylindrical-double conical, barrel and step-double conical horns were found to be significantly larger but the factor of safety was observed to be much lesser, therefore those designs were considered inappropriate in terms of operating life. Circular hollow exponential horn was observed to provide $4.3 \%$ more amplitude of vibration at tool end with slightly more factor of safety ( 1.75 $\%)$ as that of step design. This horn design can be given preference instead of step horn if high vibration amplification is desired with nearly similar operating life. The multistep-conical horn was observed to provide $17.06 \%$ more safety factor and operating life with slightly more vibration amplification. The performance of step-conical, step-Bezier, double conical, step-catenoidal, cylindrical-catenoidal, cylindrical Bezier, multistep and multistep-conical horns were observed to be much better and balanced in terms of vibration amplification and factor of safety. However, the vibration amplification achieved by step-conical, step-catenoidal and step-Bezier horns were observed to be very close to step horn design with a difference of only $0.06 \%, 2.81 \%$ and $2.69 \%$ respectively, with $35.8 \%, 65.95 \%$ and 36.96 $\%$ respectively, higher factor of safety, thus operating life. 
All ultrasonic horns, considered in this research, were designed to have natural frequencies in the axial direction distinct from operating frequency of $20 \mathrm{kHz}$, therefore stresses developed were lesser and factor of safety was higher. When they would be designed with natural frequency close to $20 \mathrm{kHz}$ to operate at resonant condition, their stresses would become large and factor of safety along with operating life would become lesser.

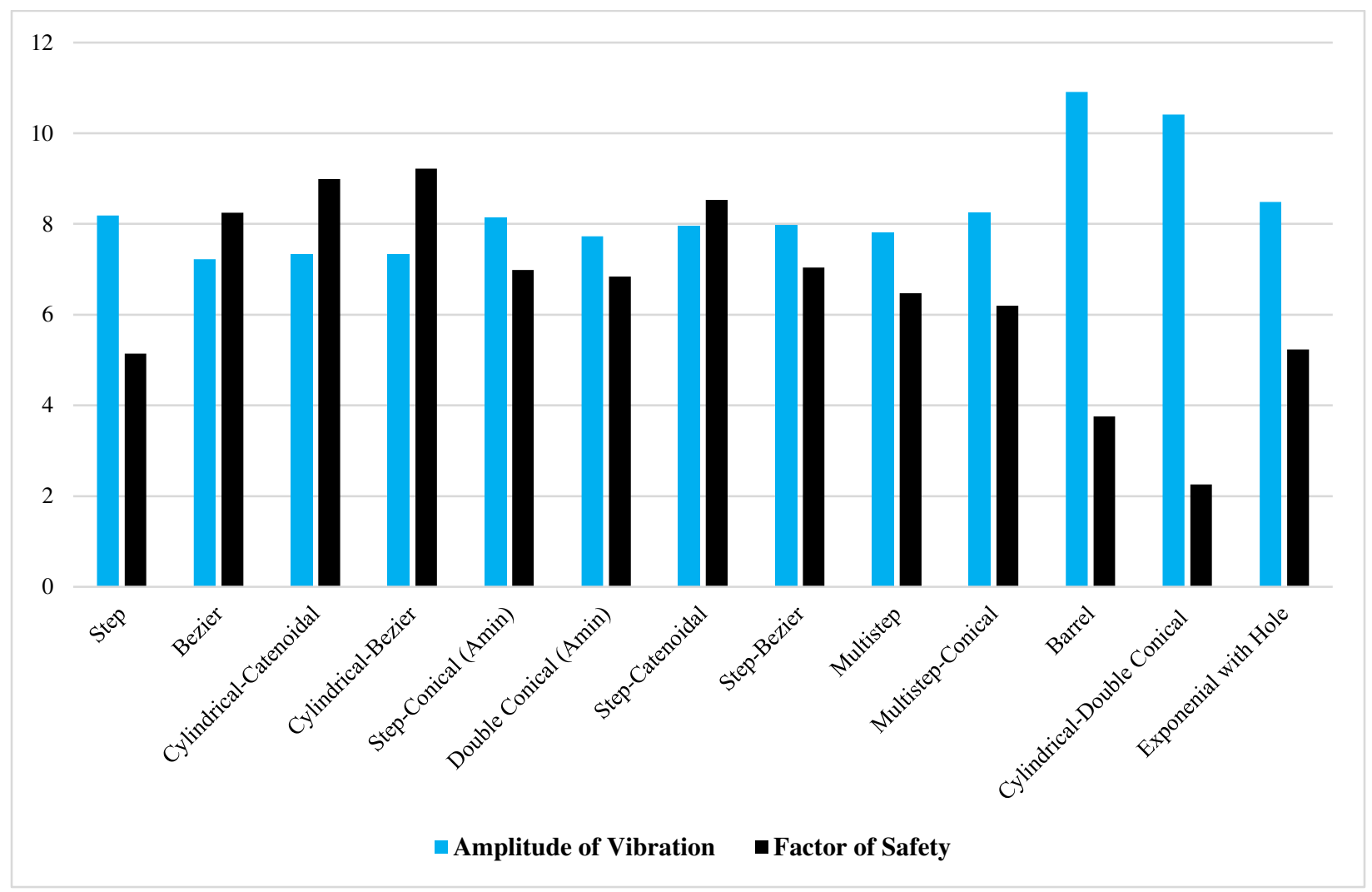

Fig. 17. Comparison of vibration amplitude and factor of safety for better ultrasonic horn designs

\section{Validation of Results}

Ultrasonic horns evaluated in this research were observed to fulfil the machining requirement of soft and brittle Nomex Honeycomb core composite, due to attaining at least $6 \mu \mathrm{m}$ amplitude of vibration at tool end. Horns were designed with resonant frequencies away from operating frequency of ultrasonic system to fulfil minimum requirements of Nomex processing. The correctness of the finite element analysis results and methodology of current research was validated with the work of other researchers. Ultrasonic Horns considered by Rani et al., Wang et al., Razavi et al, Kumar et al and Roy et al. were remodeled and finite element analysis was performed to determine amplitude of vibration, Von Mises stresses and resonant frequency [12, 15, 25, 40, 56]. Finite element analysis results were compared with experimental data of Rani et al, Razavi et al and Wang et al, and simulation outcomes of Roy et al. and Kumar et al under their specific operating conditions and input parameters, respectively. The finite element analysis results were found to be in good agreement with data of past research works as shown in Fig. 18.

The variation between research data of Wang et al and current finite element model results were observed to be $4.7 \%$ and $6.6 \%$ for Bezier and catenoidal profiles respectively. Minor difference in vibration amplification was observed owing to consideration of ultrasonic horn for simulation work in current research work, whereas Wang et al considered integrated horn and transducer system for simulation and experimentation purposes. This indicated that attaching and not attaching the transducer with ultrasonic horn would not have significant influence on the correctness of finite element analysis results. Therefore, numerous researchers considered ultrasonic horns only for carrying out modal and harmonic analysis $[6,7,13,15,19,20,25,29,33,36,40,41,48,49,55,57]$. 
For operating conditions and design parameters of Razavi et al. Kumar et al and Roy et al, the numerical results of the present finite element model were observed to be very close to their research outcomes for five element hybrid horn, Bezier horns and exponential circular hollow horn respectively $[25,40,56]$. The finite element model results of present research work were observed to be in good agreement with the data of Rani et al. [15] for catenoidal, Gaussian and cubic Beziers horns with the exception of step design, due to its capability of providing largest magnification factor among standard ultrasonic horns as reported by various researchers $[6,7,12,21,40]$. This further validates the effectiveness and correctness of finite element methods used in current research work.

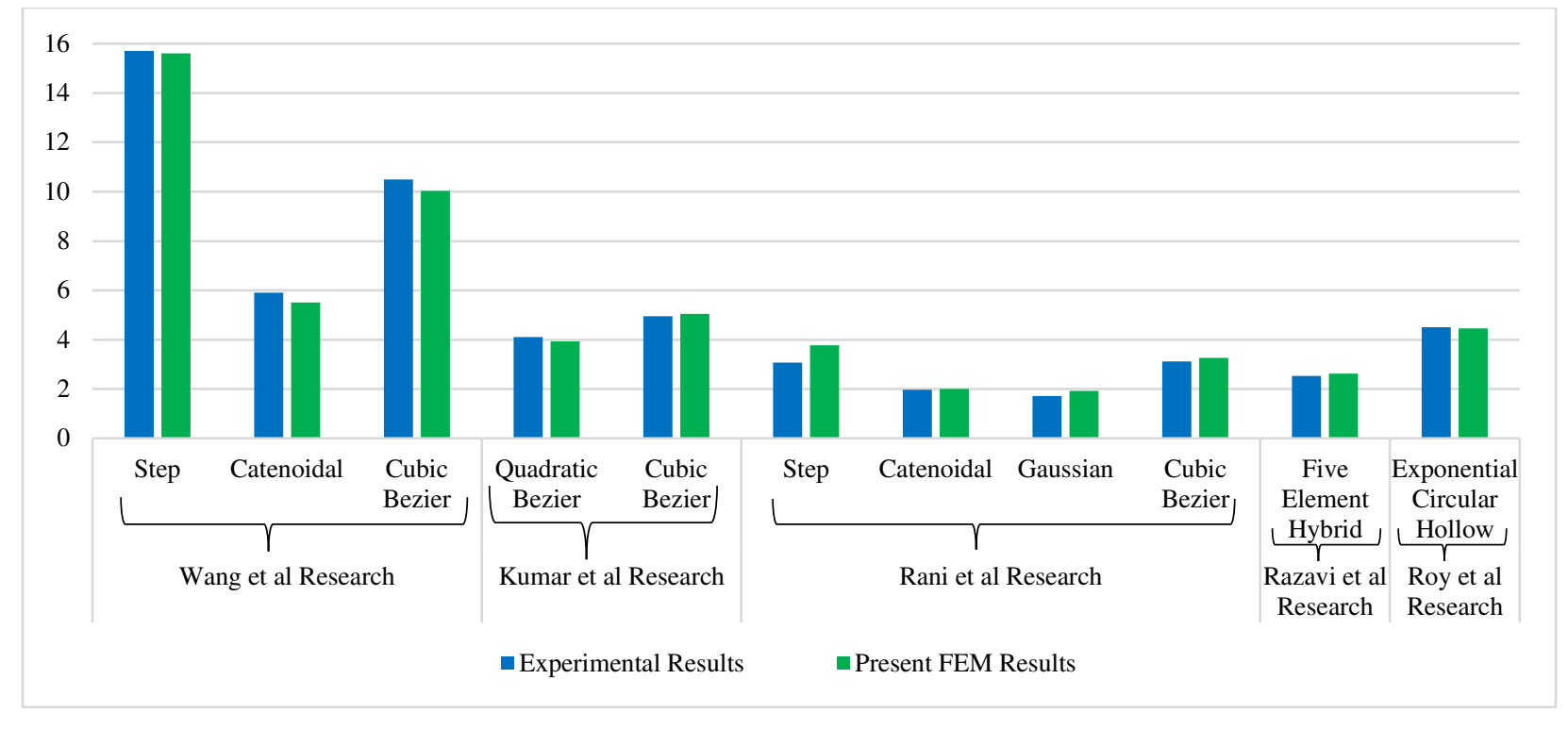

Fig. 18. Validation of Magnification factor

\section{Conclusions and future recommendations}

\subsection{Conclusions}

This research was focused on the design evaluation of state of the art ultrasonic horns to achieve higher vibration amplification and minimum stresses for ultrasonic assisted machining of soft and brittle composites such as Nomex honeycomb core. For this purpose, existing ultrasonic horns were designed along with some new combinations of standard profiles for same length, transducer and tool end diameters and operating conditions. The investigations were carried out by performing vibration analysis on ultrasonic horns made of steel in terms of modal frequency, amplitude of vibration and stresses. The simulation results showed good agreement of modal and harmonic analysis with the theoretical expectations and previous research. Based on numerical investigations, the performance of step hybrid designs was observed to be extremely well, specially step-catenoidal and step-Bezier designs in terms of vibration amplification, factor of safety and operating life. Among all ultrasonic horn designs considered in this research, only multistep-conical and circular hollow exponential horns were observed to achieve vibration amplification, factor of safety and operating life higher than commonly used step design. After numerical computations, the following conclusions were made regarding the design performance of state of the art ultrasonic horn designs.

- Among standard ultrasonic horns, Bezier and step horns can be used for higher vibration amplification and operating life.

- If more vibration amplification is desired with nearly similar operating life as that of step design, then circular hollow exponential horn would be a better choice.

- If higher factor of safety, operating life and vibration amplification are required, then multistep-conical horn can be used for ultrasonic vibration assisted machining applications.

- If reasonably good vibration amplification is desired with high factor of safety and operating life, then cylindricalcatenoidal and cylindrical-Bezier ultrasonic horns would be a good choice. 
- For high vibration amplification, factor of safety and operating life, step-conical, step-catenoidal and step-Bezier ultrasonic horns should be used.

- To achieve higher amplitude of vibration at the tool end, cylindrical-double conical, multistep-conical and barrel horns can be used for ultrasonic machining applications.

\subsection{Future Recommendations}

The design of ultrasonic horn for rotary ultrasonic machining system to achieve high vibration amplification and low stresses is extremely important in order to improve the machining quality of advanced materials. There is a huge scope of further research in this emerging field such as

- There is a room to optimize step-catenoidal, step-Bezier and step-conical ultrasonic horns for higher vibration amplification by varying the length and location of catenoidal, Bezier and conical profiles respectively.

- The lengths of catenoidal, Bezier and exponential profiles may be varied along with the length of cylindrical portion to optimize cylindrical hybrid horns.

- Circular holes may also be incorporated in standard ultrasonic horn designs to analyze their performance.

- The suggested ultrasonic horns may be manufactured to incorporate in rotary ultrasonic machining system and experimentations would be carried out on soft and brittle composites to assess machining quality.

- Other materials like aluminum, monal, tantalum and titanium can be chosen for ultrasonic horns to assess their performance.

\section{Declarations}

\section{Ethical Approval}

The manuscript is in compliance with ethical standards.

\section{Consent to Participate}

NA

\section{Consent to Publish}

\section{NA}

\section{Authors Contributions}

Khurram Hameed Mughal Conceptualization; Data curation; Formal analysis; Investigation; Methodology; Project administration; Software; Validation; Visualization; Writing - original draft.

Muhammad Asif Mahmood Qureshi Conceptualization; Methodology; Project administration; Resources; Software; Supervision; Validation; Visualization; Roles/Writing - original draft; Writing - review \& editing.

Asif Ali Qaiser Methodology; Project administration; Resources; Supervision; Validation; Writing - review \& editing.

Fazal Ahmad Khalid Project administration; Resources; Writing - review \& editing.

\section{Funding}

No funding was received to assist with the preparation of this manuscript. 


\section{Competing Interests}

The authors have no relevant financial or non-financial interests to disclose.

\section{Availability of data and materials}

The authors are ready to ensure the availability of data and materials, when required.

\section{References}

1. Choon Chiang Foo, Gin Boay Chai, Leong Keey Seah, Mechanical properties of Nomex material and Nomex honeycomb structure. Composite Structures, 2007. 4(80): p. 588-594.

2. Li, The Development and Application of the Nomex Honevcomb in Russia Aerospace Area. Journal of Materials Engineering, 1995.

3. Cheng Yong et al., Comparative study of ultrasonic cutting and traditional CNC machining of Nomex honeycomb core structure parts, in National Composites Conference. 2012.

4. Weilong Cong, Zhijian Pei, Process of Ultrasonic Machining, Handbook of Manufacturing Engineering and Technology. 2015: Springer.

5. Shahzad Ahmad, Jianfu Zhang, Pingfa Feng, Dingwen Yu, Zhijun Wu, Ke Ma, Processing Technologies for Nomex Honeycomb Composites (NHCs): a critical review. Composite Structures, 2020.

6. S.G. Amin, M.H.M. Ahmed, H.A. Youssef, Computer-aided design of acoustic horns for ultrasonic using finite-element analysis. Journal of Materials Processing Technology, 1995(55): p. 254-260.

7. Yu Pang, Pingfa Feng, Jianfu Zhang, Yuan Ma, \& Qiaoli Zhang, Frequency coupling design of ultrasonic horn with spiral slots and performance analysis of longitudinal-torsional machining characteristics. The International Journal of Advanced Manufacturing Technology, 2020.

8. Di Kang, Ping Zou, Hao Wu, Jingwei Duan, Wenjie Wang, Study on ultrasonic vibration-assisted cutting of Nomex honeycomb cores. The International Journal of Advanced Manufacturing Technology, 2019.

9. Jiansong Sun, Z.D., Xuanping Wang, Yidan Wang, Yan Qin, Renke Kang, Simulation and experimental study of ultrasonic cutting for aluminum honeycomb by disc cutter. Ultrasonics, 2020. 103.

10. G. Amza and D. Drimer, The design and construction of solid concentrators for ultrasonic energy. Ultrasonics, 1976.

11. Stewart Sherrit, B.P.D., Yoseph Bar-Cohen, Modeling of Horns for Sonic/Ultrasonic Applications, in IEEE Ultrasonics Symposium. 1999.

12. D. A. Wang, W. Y. Chuang, K. Hsu, H. T. Pham, Design of a Bézier-profile horn for high displacement amplification. Ultrasonics, 2011(51): p. 148-156.

13. K. H .W. Seah , Y.S. Wong, L.C. Lee, Design of tool holders for ultrasonic machining using FEM. Journal of Materials Processing Technology, Elsevier, 1993(37): p. 801-816.

14. B. Fu, T. Hemsel, J. Wallaschek, Piezoelectric transducer design via multiobjective optimization. Ultrasonics 2006. 44: p. 747-752.

15. M. Roopa Rani, R. Rudramoorthy, Computational modeling and experimental studies of the dynamic performance of ultrasonic horn profiles used in plastic welding. Ultrasonics, 2013. 53(53): p. 763-772.

16. Lorenzo Parrini, New technology for the design of advanced ultrasonic transducers for high-power applications. Ultrasonics, 2003. 41: p. 261-269.

17. A. Iula, L. Parenti, F. Fabrizi, M. Pappalardo, A high displacement ultrasonic actuator based on a flexural mechanical amplifier. Sensors and Actuators, 2006. 125(3): p. 118-12.

18. Kuo, Kei-Lin, Design of rotary ultrasonic milling tool using FEM simulation. journal of materials processing technology, 2008. 201: p. 48-52.

19. M. Roopa Rani, K. Prakasan, R. Rudramoorthy, Studies on thermo-elastic heating of horns used in ultrasonic plastic welding. Ultrasonics, 2015(55): p. 123-132.

20. Milan Nad, Ultrasonic horn design for ultrasonic machining technologies. Applied and Computational Mechanics, 2010(4): p. 79-88.

21. Shuyu Lin, Hao Guo, Jie Xu, Actively adjustable step-type ultrasonic horns in longitudinal vibration. Journal of Sound and Vibration, 2018. 419: p. 367-379.

22. Huilin Zhou, Jianfu Zhang, Pingfa Feng, Dingwen Yu, Wanchong Cai, An output amplitude model of giant magnetostrictive rotary ultrasonic machining system considering load effect. Precision Engineering, 2019.

23. Huilin Zhou, Jianfu Zhang, Pingfa Feng, Dingwen Yu, Zhijun Wu, Design on amplitude prediction model for a giant magnetostrictive ultrasonic transducer. Ultrasonics, 2019.

24. Xiao-Bo Wang, Feng Jiao, Chong-Yang Zhao, Optimization of the longitudinal-torsional ultrasonic step-like horn design for milling tool applications via the tool load account. Journal of Vibroengineering, 2019. 21(8): p. 2250-2259. 
25. Praveen Kumar Rai, Vinod Yadava, Rabindra Kumar Patel, Design of Bezier profile horns by using optimization for high amplification. Journal of the Brazilian Society of Mechanical Sciences and Engineering, 2020.

26. M. Roopa Rani, K. Prakasan and R. Rudramoorthy, Design and simulation of a large tubular horn for ultrasonic plastic welding. International Journal of Design Engineering, 2014. 5(4): p. 344-456.

27. D. Nedelcu, V. Cojocaru, M. Nedeloni, F. Peris-Bendu, A. Ghican, Failure analysis of a Ti-6Al-4V ultrasonic horn used in cavitation erosion tests. Mechanika, 2015. 21(4): p. 272-276.

28. Kuen-Ming Shu, Jyun-Wei Chen, The Design of Acoustic Horns for Ultrasonic Aided Tube Double Side Flange Making. International Journal of Mechanical, Aerospace, Industrial, Mechatronic and Manufacturing Engineering, 2015. 9(5).

29. M. P. Satpathy, S. K. Sahoo \& S. Datta, Acoustic Horn Design and Effects of Process Parameters on Properties of Dissimilar Ultrasonic Welding Aluminium to Brass. Materials and Manufacturing Processes, 2015.

30. Xiao-Bo Wang, Feng Jiao, Chong-Yang Zhao, Optimization of the longitudinal-torsional ultrasonic step-like horn design for milling tool applications via the tool load account. Journal of Vibroengineering, 2019. 21(8).

31. S. Amini, H. Soleimanimehr, M.J. Nategh,*, A. Abudollah, M.H. Sadeghi, FEM analysis of ultrasonic-vibration-assisted turning and the vibratory tool. journal of materials processing technology, 2008. 201: p. 43-47.

32. Jilong Yang, Shijun Ji, Ji Zhao, Qiuwei He, Theoretical analysis and finite element calculation of ultrasonic horn, in IOP Conference Series: Materials Science and Engineering, ACMME. 2019, IOP Publishing.

33. Woosin Jung, Jongman Ra and Keun Park, Design Optimization of Ultrasonic Horn for Micro-Pattern Replication. International Journal of Precision Engineering and Manufacturing, 2012. 13(12): p. 2195-2201.

34. Liu Chuanshao, Wu Haoqiong, Luo Aome, Design of Longitudinal-Torsional Vibration Stepped Horn with Multiple Diagonal Slits. Applied Mechanics and Materials, 2013. 312: p. 51-54.

35. R. Dipin Kumar, M. Roopa Rani, S. Elangovan, Design and Analysis of Slotted Horn for Ultrasonic Plastic Welding. Applied Mechanics and Materials, 2014. 592-594: p. 859-863.

36. Tao Chen, Shuliang Liu, Wu Liu and ChaoqunWu, Study on a longitudinal-torsional ultrasonic vibration system with diagonal slits. Advances in Mechanical Engineering, 2017. 9(7): p. 1-10.

37. Ziad Shakeeb Al Sarraf, Design and analysis of ultrasonic horns operating in multiple vibration modes. Applied Engineering, 2018. 2 (2): p. 28-32.

38. He Xiping, Wang Yanjun, Ning Jingfeng, A high displacement ultrasonic horn with a cuboid hole in the front end, in Proceedings of 2019 16th International Bhurban Conference on Applied Sciences \& Technology (IBCAST), 2019, IEEE: Islamabad, Pakistan.

39. Xiaobo Wang, Feng Jiao, Dong Wang and Y. Niu, Development of a longitudinal-torsional ultrasonic vibration aided drilling system for drilling carbon fiber reinforced polymer materials. Journal of Mechanical Engineering Science, 2019: p. 1-11.

40. Subhankar Roy \& Jagadish, Design of a circular hollow ultrasonic horn for USM using finite element analysis. The International Journal of Advanced Manufacturing Technology, 2016.

41. Jagadish, Amitava Ray, Design and performance analysis of ultrasonic horn with a longitudinally changing rectangular cross section for USM using finite element analysis. Journal of the Brazilian Society of Mechanical Sciences and Engineering, 2018. 40(359).

42. Karl-Robert Deibel, Konrad Wegener, Methodology for shape optimization of ultrasonic amplifier using genetic algorithms and simplex method. Journal of Manufacturing Systems, 2013. 32: p. 523- 528.

43. Chun-fang Xiao, Han Bing, Research and Design of Ultra-long Ultrasonic Horn. Journal of The Institution of Engineers (India): Series C, 2018.

44. Chun-fang Xiao, Han Bing, Research and Design of Ultra Long Ultrasonic Horn. Research \& Development in Material Science, 2018. 7(3).

45. R. Naseri, K. Koohkan, M. Ebrahimi, F. Djavanroodi, H. Ahmadian, Horn design for ultrasonic vibration-aided equal channel angular pressing. International Journal of Advanced Manufacturing Technology, 2016.

46. Dhirendra Pratap Singh, Sanjay Mishra, Rajesh Kumar Porwal. Modal Analysis of Ultrasonic Horn using Finite Element Method. in 9th International Conference of Materials Processing and Characterization, ICMPC. 2019. Elsevier.

47. Ma Ke, Jianfu Zhang, Feng Pingfa, Wu Zhijun, Yu Dingwen, Shahzad Ahmad, Design and Implementation of a Mini Ultrasonic Cutting System for Nomex Honeycomb Composites, in Proceedings of 2019 16th International Bhurban Conference on Applied Sciences \& Technology (IBCAST) 2019: Islamabad, Pakistan.

48. Sergei L. Peshkovsky, Alexey S. Peshkovsky, Matching a transducer to water at cavitation: Acoustic horn design principles. Ultrasonics Sonochemistry, 2007. 14: p. 314-322.

49. Vinod Yadava and Aniruddha Deoghare, Design of horn for rotary ultrasonic machining using the finite element method. International Journal of Advanced Manufacturing Technology, 2008. 39: p. 9-20.

50. Zhiqiang Fu, Xiaojun Xian, Shuyu Lin, Chenghui Wang, Wenxu Hu, Guozheng Li, Investigations of the barbell ultrasonic transducer operated in the full-wave vibrational mode. Ultrasonics, 2012. 52: p. 578-586.

51. Hongbing Wang, C.S., Finite Element Analysis and Test of an Ultrasonic Compound Horn. World Journal of Engineering and Technology, 2017. 5: p. 351-357.

52. Jiyan Lin and Shuyu Lin, Study on a Large-Scale Three-Dimensional Ultrasonic Plastic Welding Vibration System Based on a Quasi-Periodic Phononic Crystal Structure. Crystals, 2020. 10(21).

53. Kuen-Ming Shu, Wen-Hsiang Hsieh and Hoa-Shen Yen, On the design and analysis of acoustic horns for ultrasonic welding. Transactions of the Canadian Society for Mechanical Engineering, 2013. 37(3). 
54. J. Woo, Y. Roh, K. Kang, S. Lee. Design and construction of an acoustic horn for high power ultrasonic transducers. in Proceedings of 2006 IEEE Ultrasonics, Symposium. 2006.

55. Young-Jae Choi, Kyung-Hee Park, Yun-Hyuck Hong, Kyeong-Tae Kim, Seok-Woo Lee, and Hon-Zong Choi, Effect of Ultrasonic Vibration in Grinding; Horn Design and Experiment. International Journal of Precision Engineering and Manufacturing, 2013. 14(11): p. 1873-1879.

56. Hamed Razavi, Mohammad Keymanesh, Iman Fakhari Golpayegani, Analysis of free and forced vibrations of ultrasonic vibrating tools, case study: ultrasonic assisted surface rolling process. The International Journal of Advanced Manufacturing Technology, 2019. 103: p. 2725-2737.

57. S. Kumar, W. Ding, Z. Sun, \& C. S. Wu, Analysis of the dynamic performance of a complex ultrasonic horn for application in friction stir welding. The International Journal of Advanced Manufacturing Technology, 2018.

58. Tinghao Tang, Huifeng Zheng, Sangsang Yu, Jiangming Lv, Design of Ultrasonic Horn for Soft Magnetic Ferrite De-burring. Applied Mechanics and Materials, 2013. 278-280: p. 197-201.

59. Fujun Wang, Xingyu Zhao, Dawei Zhang, Yimin Wu, Development of novel ultrasonic transducers for microelectronics packaging. journal of materials processing technology, 2009. 209: p. 1291-1301.

60. J. J. Coy, F. S. Tse, Synthesis of solid elastic horns. Journal of Engineering for Industry, ASME, 1974. 96(2): p. 627-632.

61. Ioan Călin Roúca, Sergiu T. Chiriacescua, Nicolae Constantin CreĠu, Ultrasonic horns optimization, in Physics procedia, International Congress on Ultrasonics, Universidad de Santiago de Chile,. 2009. p. 1033-1040.

62. Ioan-Calin Rosca, Mihail-Ioan Pop, Nicolae Cretu, Experimental and numerical study on an ultrasonic horn with shape designed with an optimization algorithm. Applied Acoustics, 2015. 95: p. 60-69.

63. Maria Violeta Guiman and Ioan Calin Rosca, A New Approach on Vibrating Horns Design. Shock and Vibration, 2017.

64. Iulian Stanasel, Traian Buidos and Florin Blaga, Design and FEM simulation of ultrasonic welding horn. Nonconventional Technologies Review, 2014.

65. He Xiping and Zhang Haidao, Analytical and experimental study of an ultrasonic horn with a bend angle. Journal of Vibration and Control, 2017. 2017: p. 1-12.

66. W.P. Mason and R.F. Wick, A BariumTitanateTransducer Capable of Large Motion at an Ultrasonic Frequency. The Journal of the Acoustical Society of America, 1951. 23(2): p. 209-214.

67. Tao He, Xin-Quan Ye, Yang Zhao, Optimization design for ultrasonic horn with large amplitude based on genetic algorithm. JVE International Ltd. Journal of Vibroengineering, 2015. 17(3): p. 1157-1168.

68. Djo Bakadiasa Kabongo, YA Gang, Experimental Design and Optimization of Conical Horn of Ultrasonic Amplitude. International Journal of Advanced Engineering Research and Science (IJAERS), 2018. 5(6).

69. Yundian Zhang, Zhongqiu Ma., The Study of Resonant Frequency of Horn and Circular Tool of Ultrasonic Milling System. Applied Mechanics and Materials, 2014. 628: p. 283-286.

70. S.S. Rao, Mechanical Vibrations. 6th ed. 2016: Pearson. 1152.

71. Gordon Webster. Acoustical Impedance, and the Theory of Horns and of the Phonograph. in Proceedings of the National Academy of Sciences of the United States of America. 1919. National Academy of Sciences.

72. Boresi AP, Schmidt RJ, Advanced Mechanics of Materials. 6th ed ed. 2003, New Jersey, NJ, USA: John Wiley and Sons.

73. Liu, Y and G. Glass. Choose the best element size to yield accurate FEA results while reduce FE model's complexity. Br. J. Eng. Technol, 2013. 1: p. 13-28.

74. S. Moaveni, Finite element analysis theory and application with ANSYS. 3/e ed. 2011: Pearson Education.

75. Daryl L. Logan, A First Course in the Finite Element Method. 6th ed. 2017: Cengage Learning.

76. S. Graham Kelly, Mechanical Vibrations; Theory and Applications. 1st ed. 2011: Cengage Learning. 672.

77. R.C. Hibbeler, Mechanics of Materials. 10 ed. 2016: Pearson. 896.

78. Joseph Edward Shigley, Richard G. Budynas, J. Keith Nisbett, mechanical engineering design. tenth edition 2015, New York, USA: McGraw-Hill 1082. 


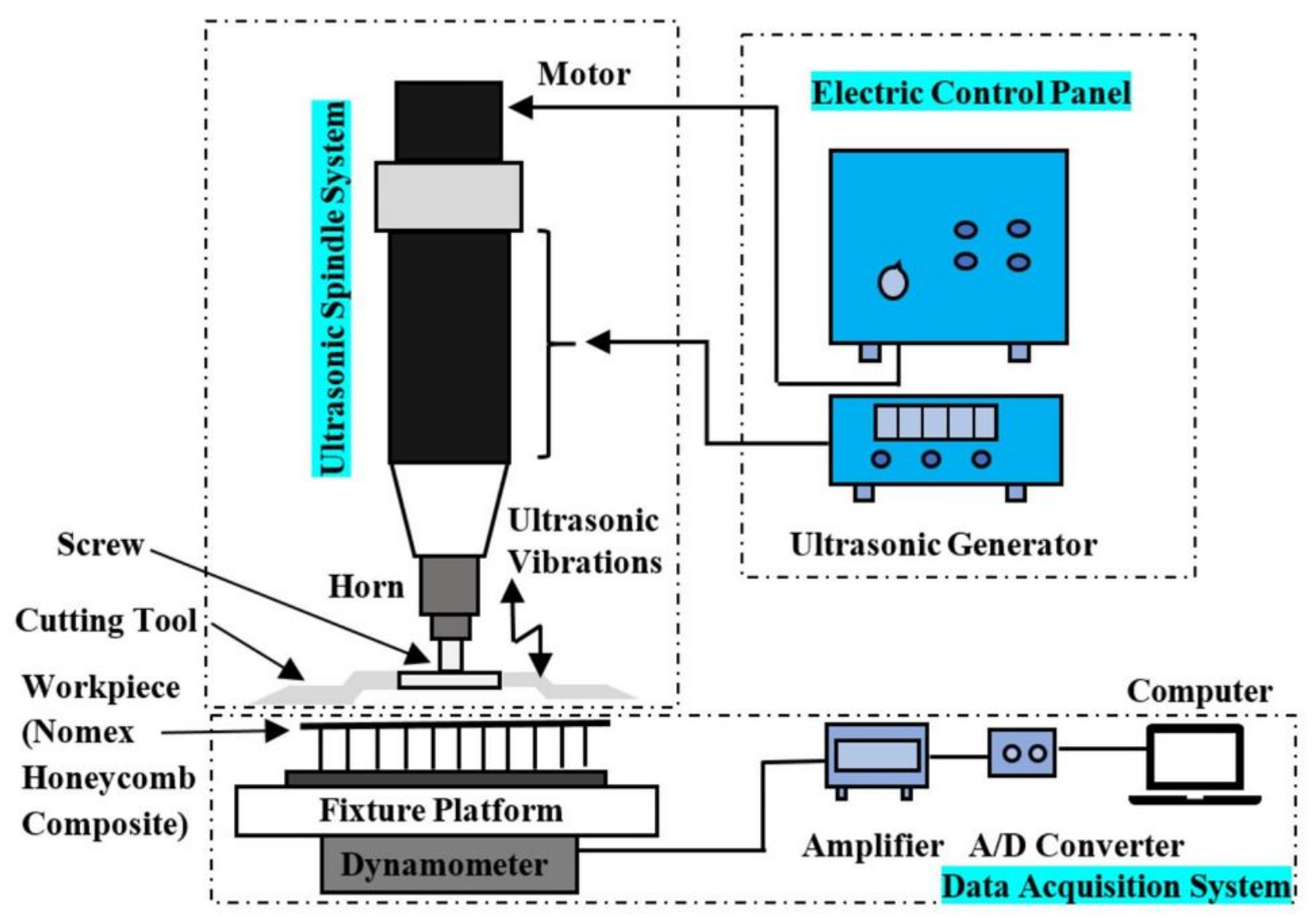

Figure 1

Schematic of Ultrasonic Assisted Cutting System 

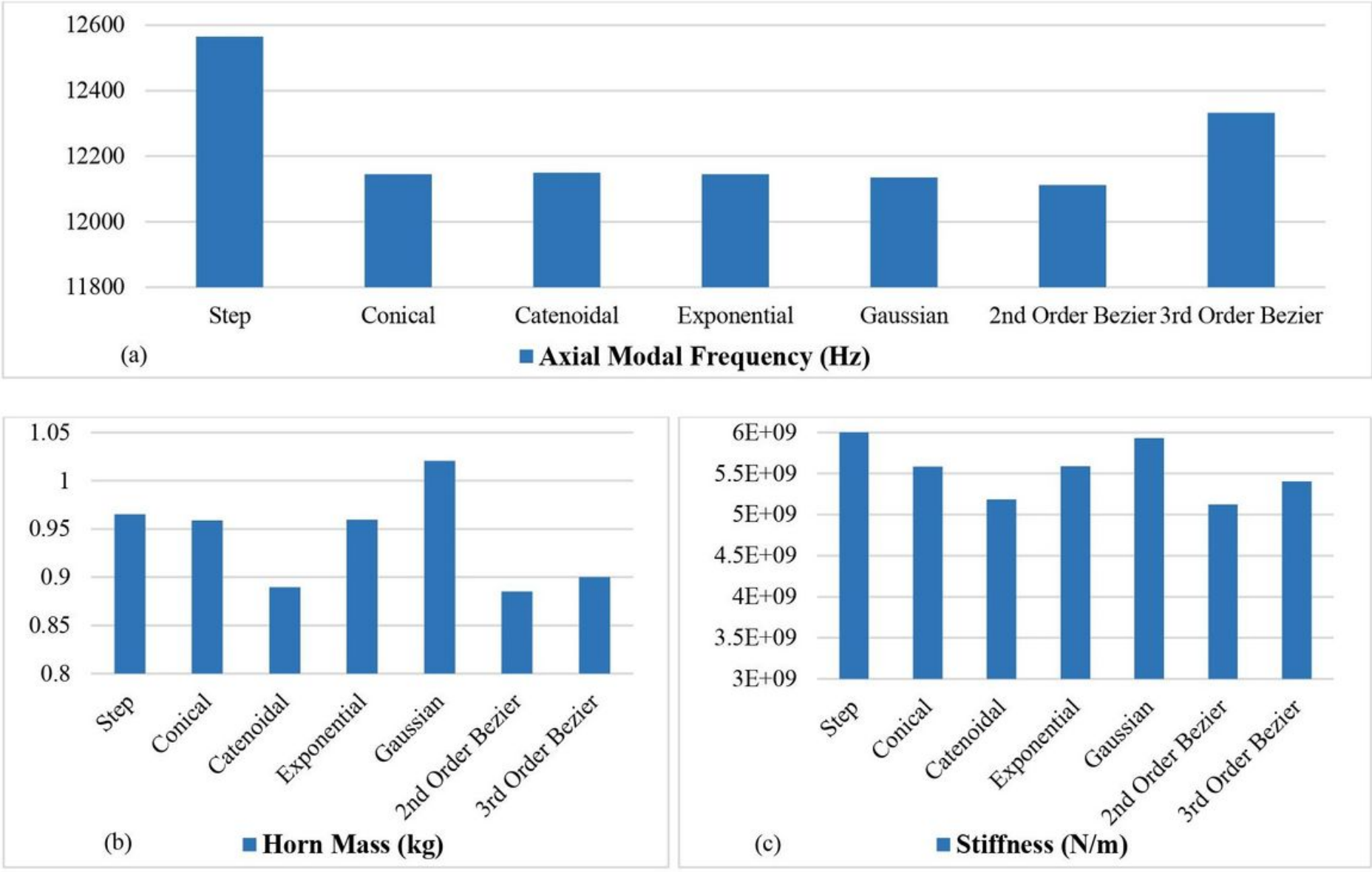

(c)

$$
\text { - Stiffness (N/m) }
$$

\section{Figure 2}

Comparison of standard ultrasonic horn designs in terms of a) axial modal frequency, b) mass, c) axial stiffness 

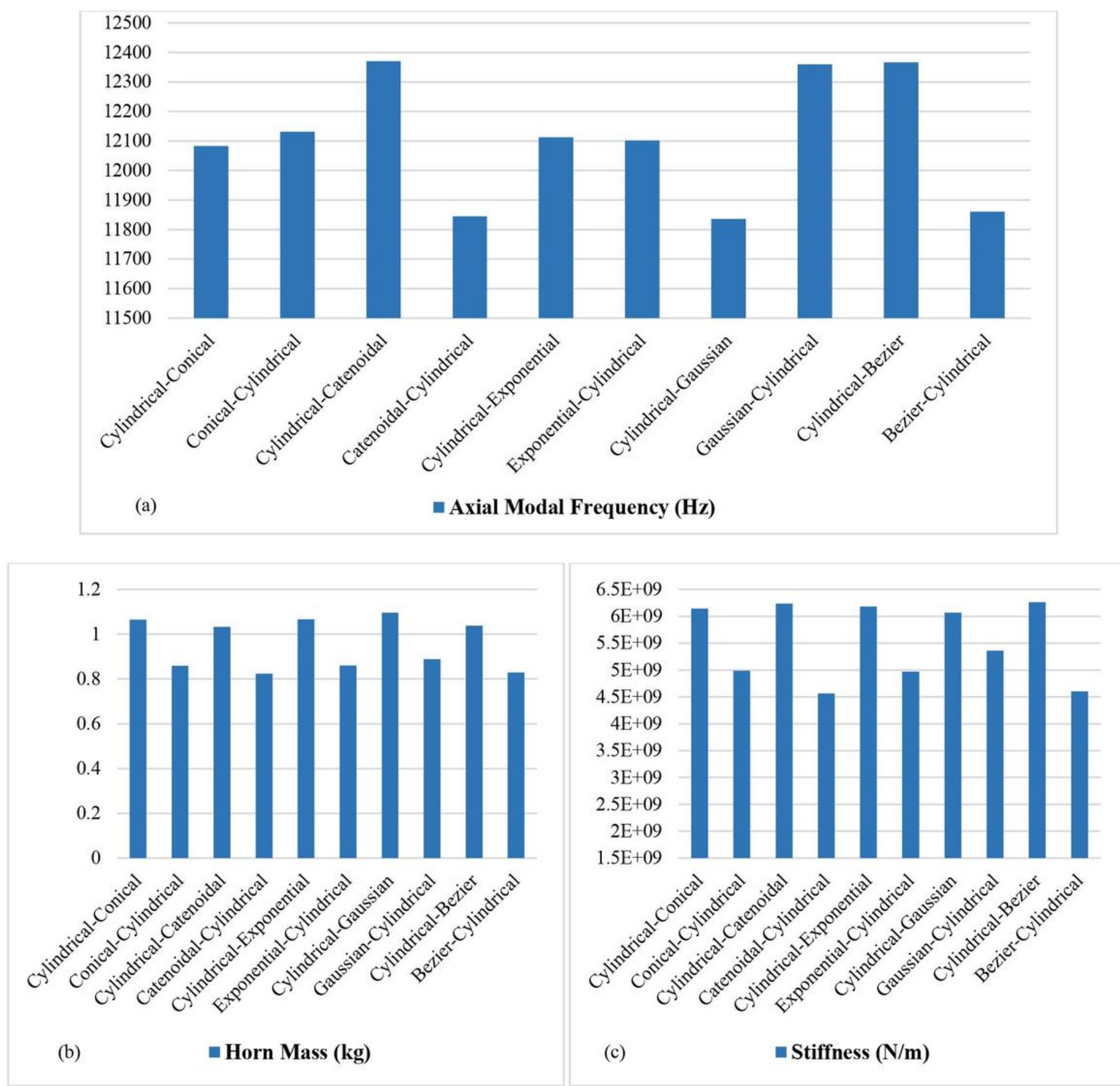

\section{Figure 3}

Comparison of cylindrical hybrid ultrasonic horn designs in terms of a) axial modal frequency, b) mass, c) axial stiffness 

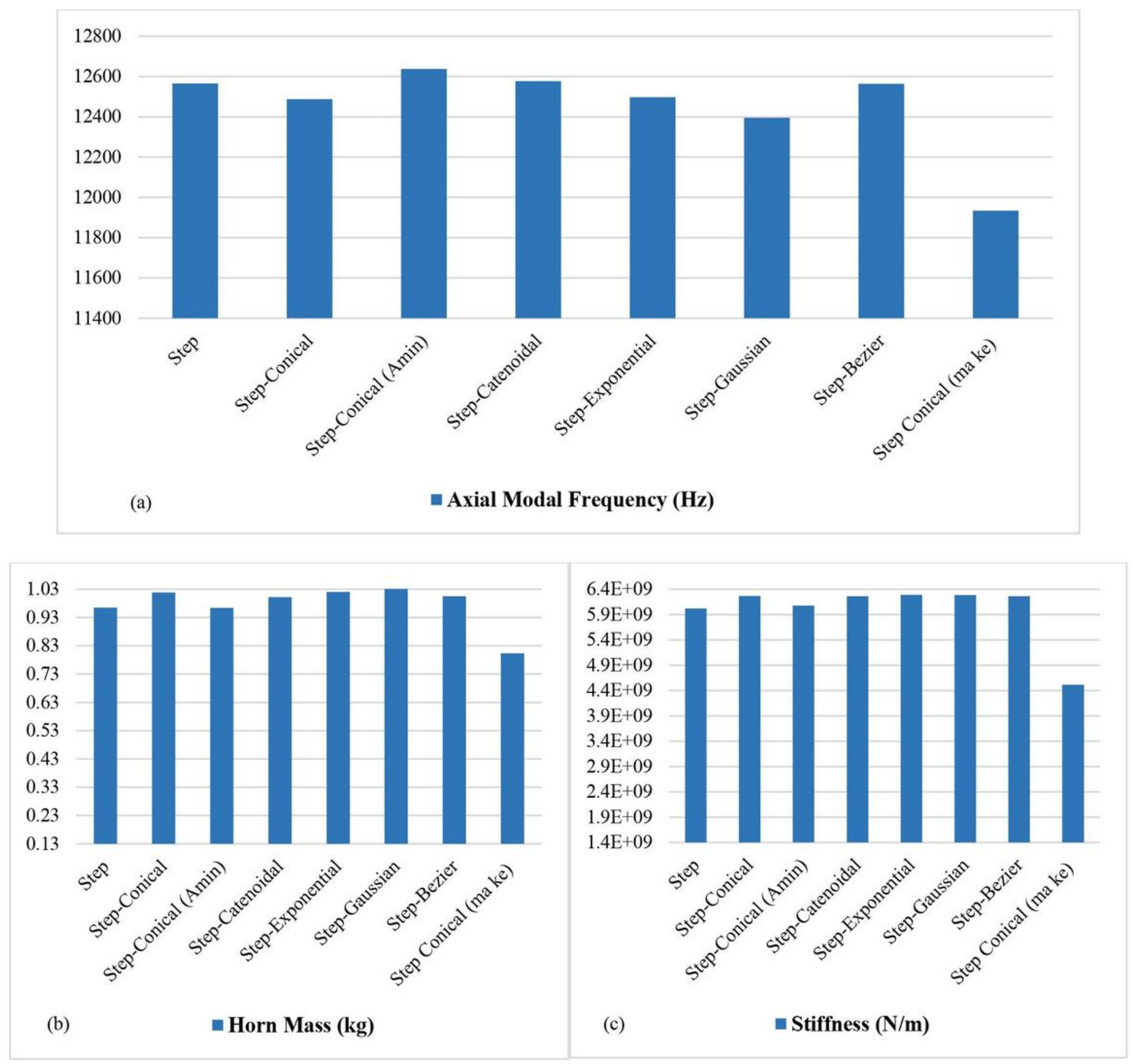

Figure 4

Comparison of step hybrid ultrasonic horn designs in terms of a) axial modal frequency, b) mass, c) axial stiffness 

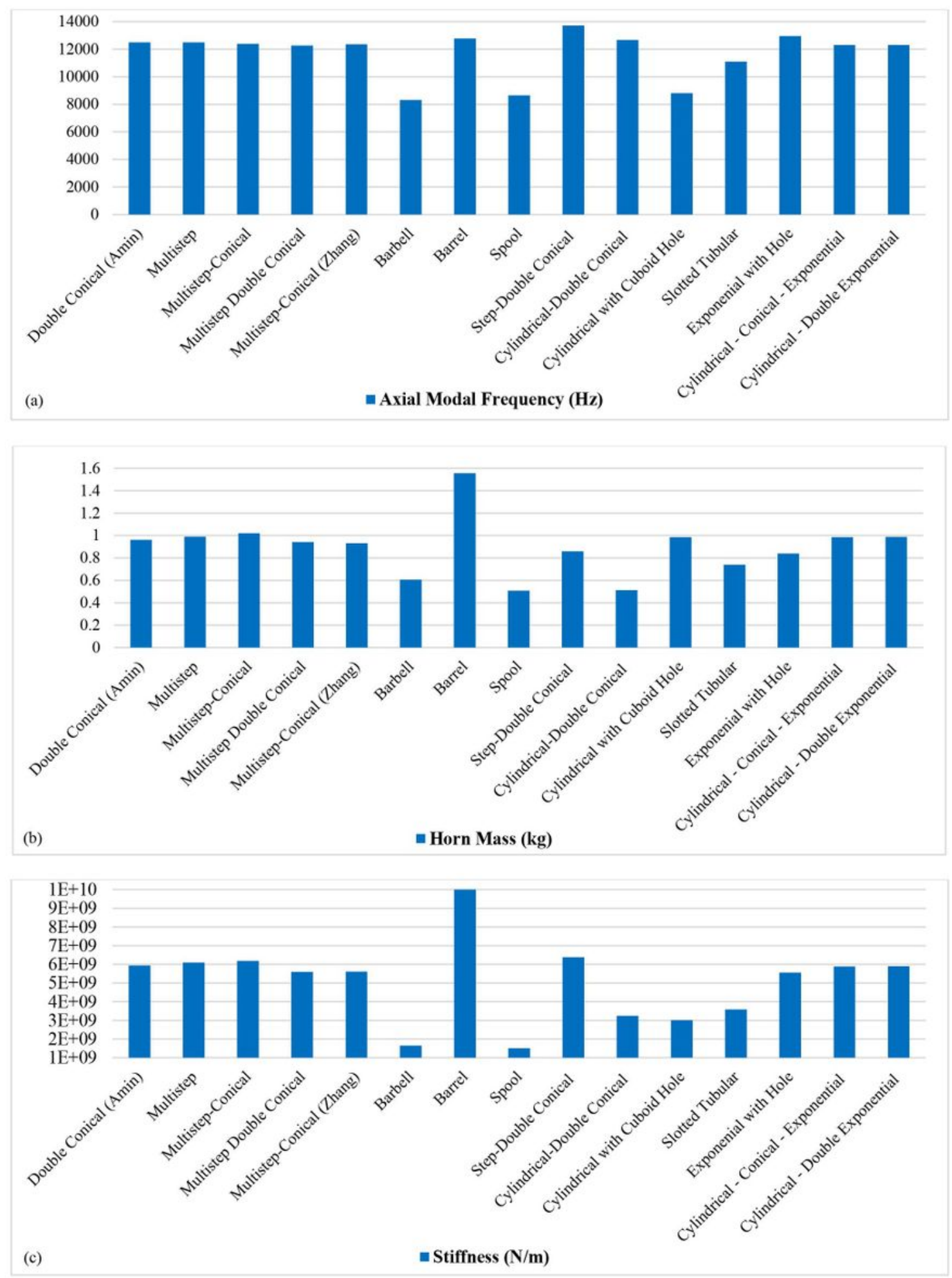

\section{Figure 5}

Comparison of specialized complex hybrid ultrasonic horn designs in terms of a) axial modal frequency, b) mass, c) axial stiffness 

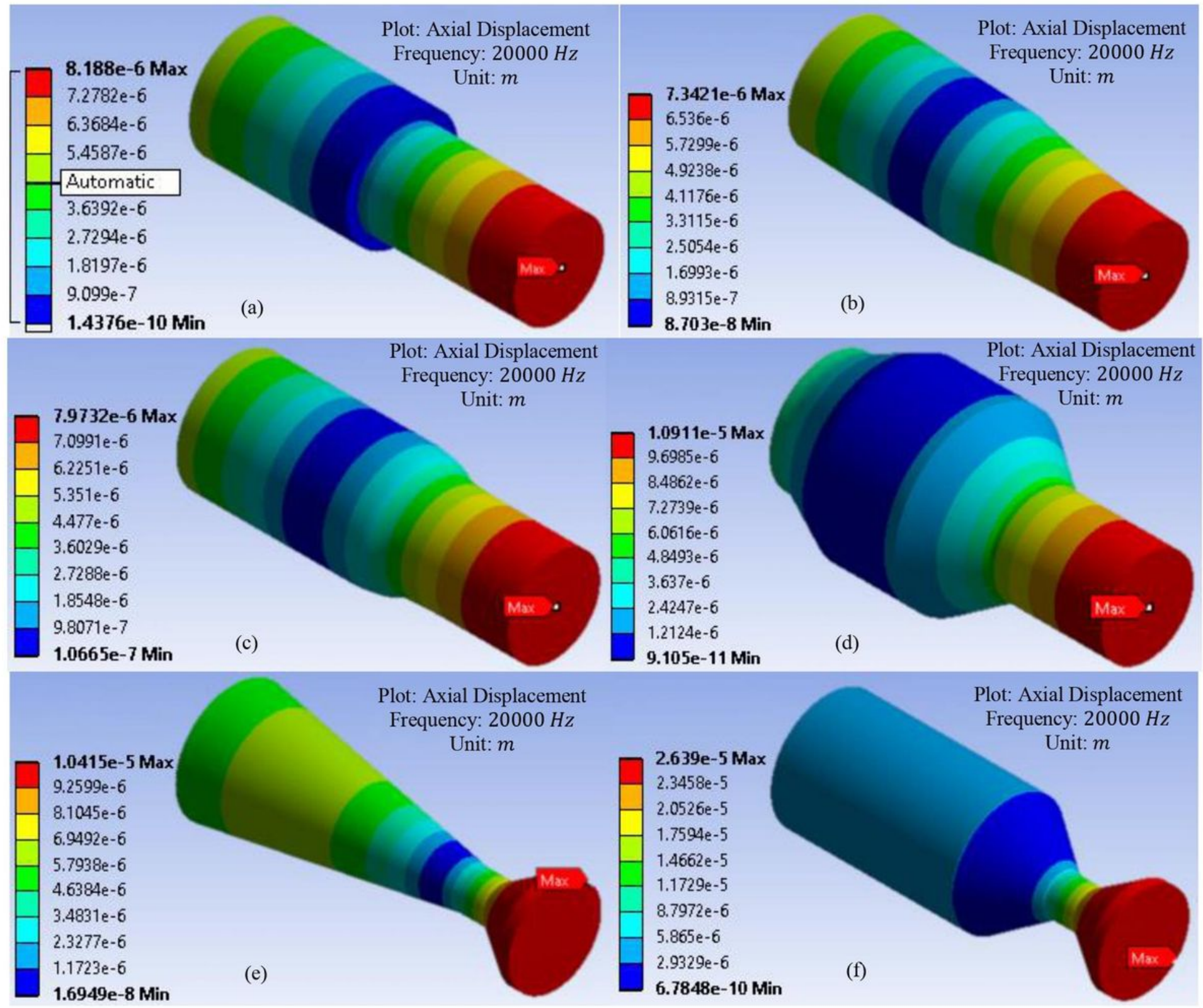

\section{Figure 6}

Contour plots of axial displacement indicating vibration amplitudes for a) step horn, b) step-catenoidal horn, c) step-Bezier horn, d) barrel horn, e) cylindrical-double conical horn, e) step-double conical horn 


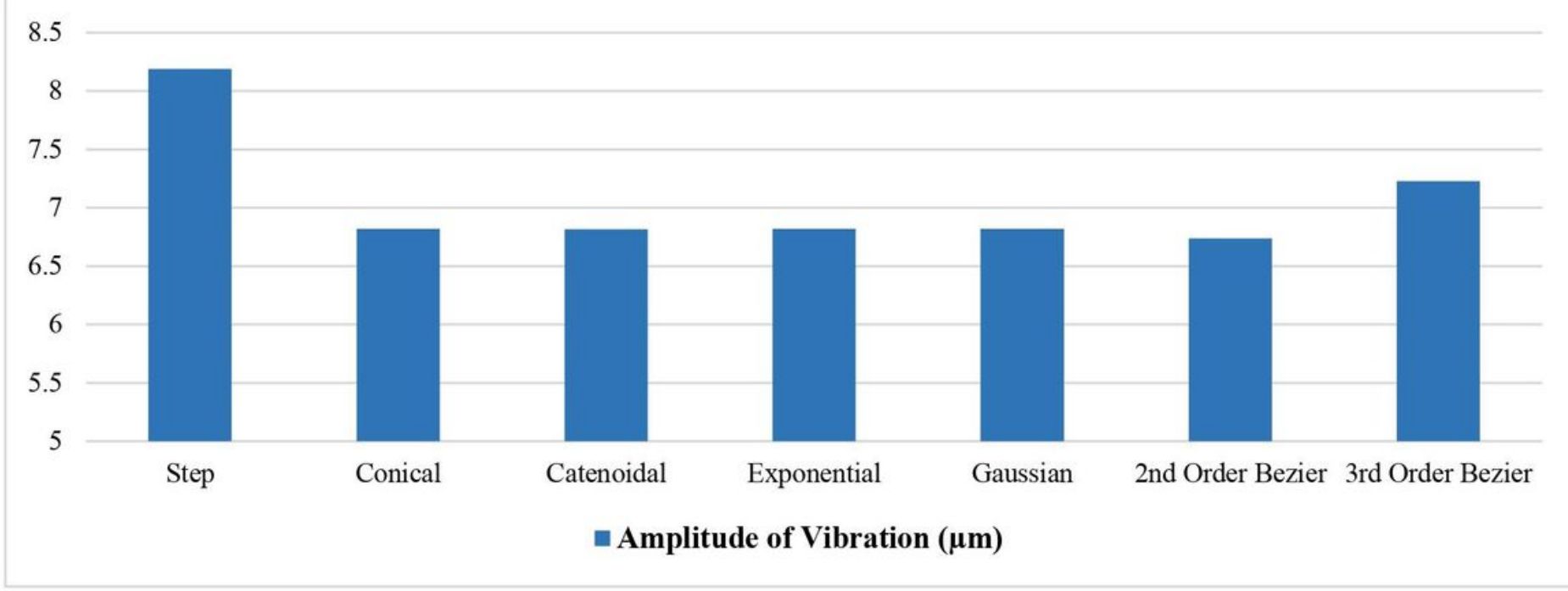

Figure 7

Comparison of amplitude of vibration for standard ultrasonic horn designs

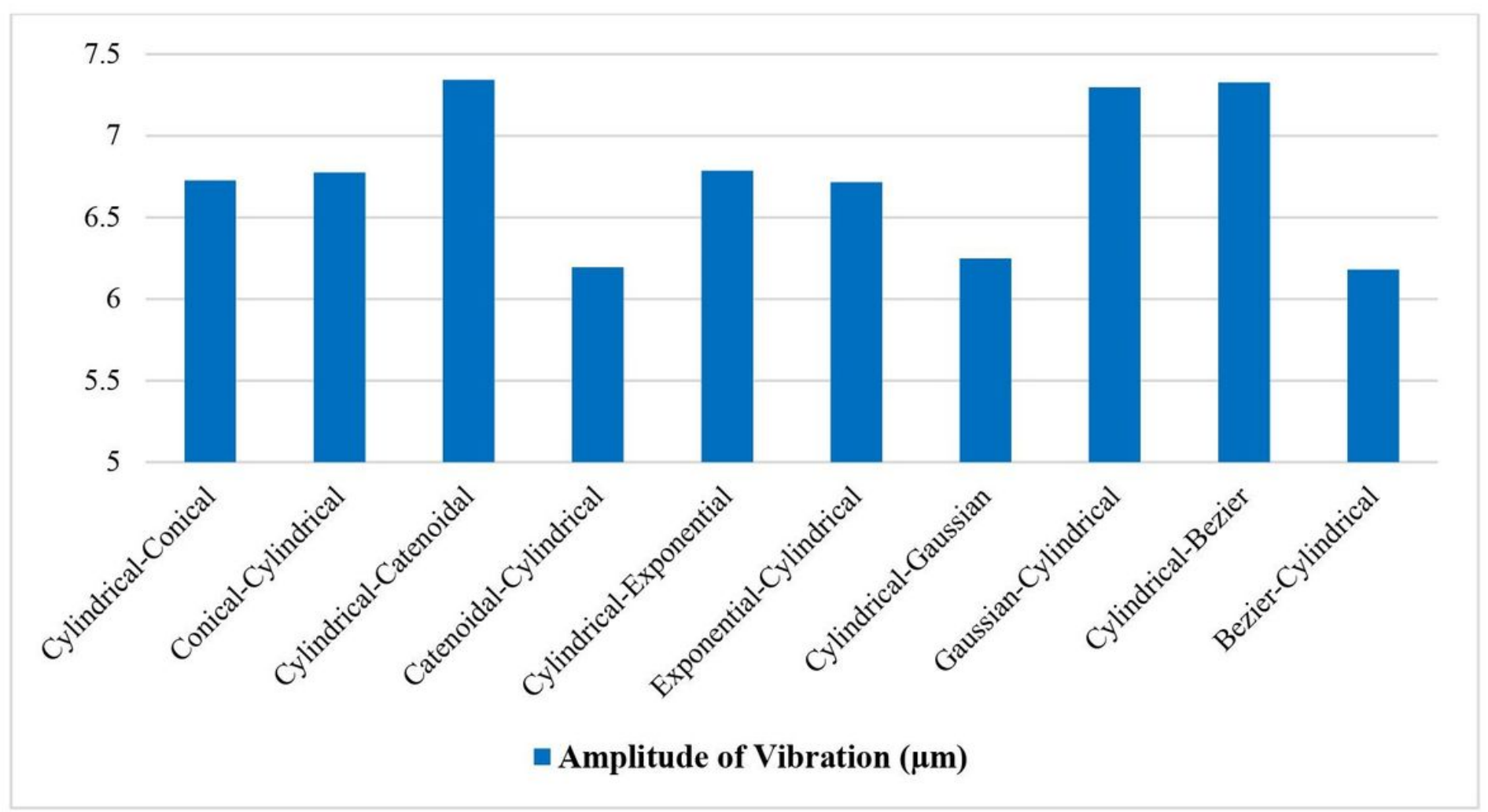

Figure 8

Comparison of amplitude of vibration for hybrid cylindrical ultrasonic horn designs 


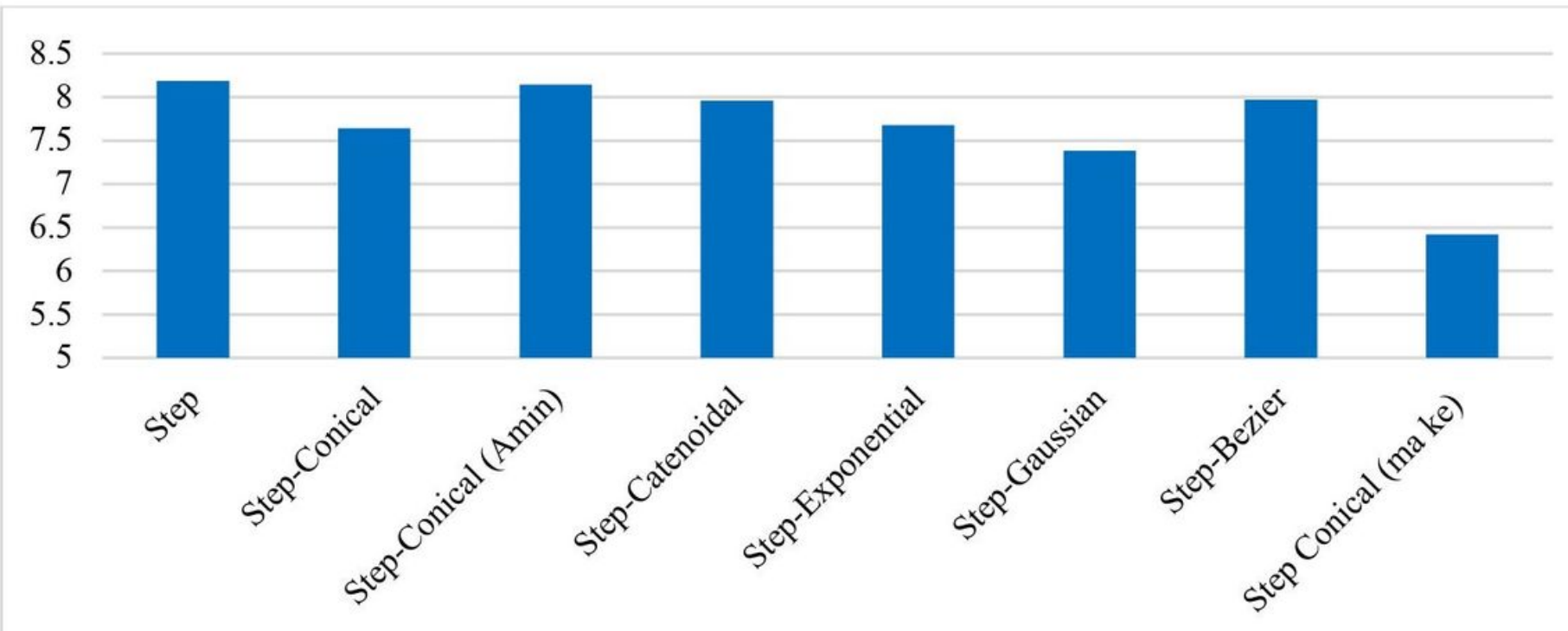

Amplitude of Vibration $(\mu \mathrm{m})$

\section{Figure 9}

Comparison of amplitude of vibration for hybrid step type ultrasonic horn designs

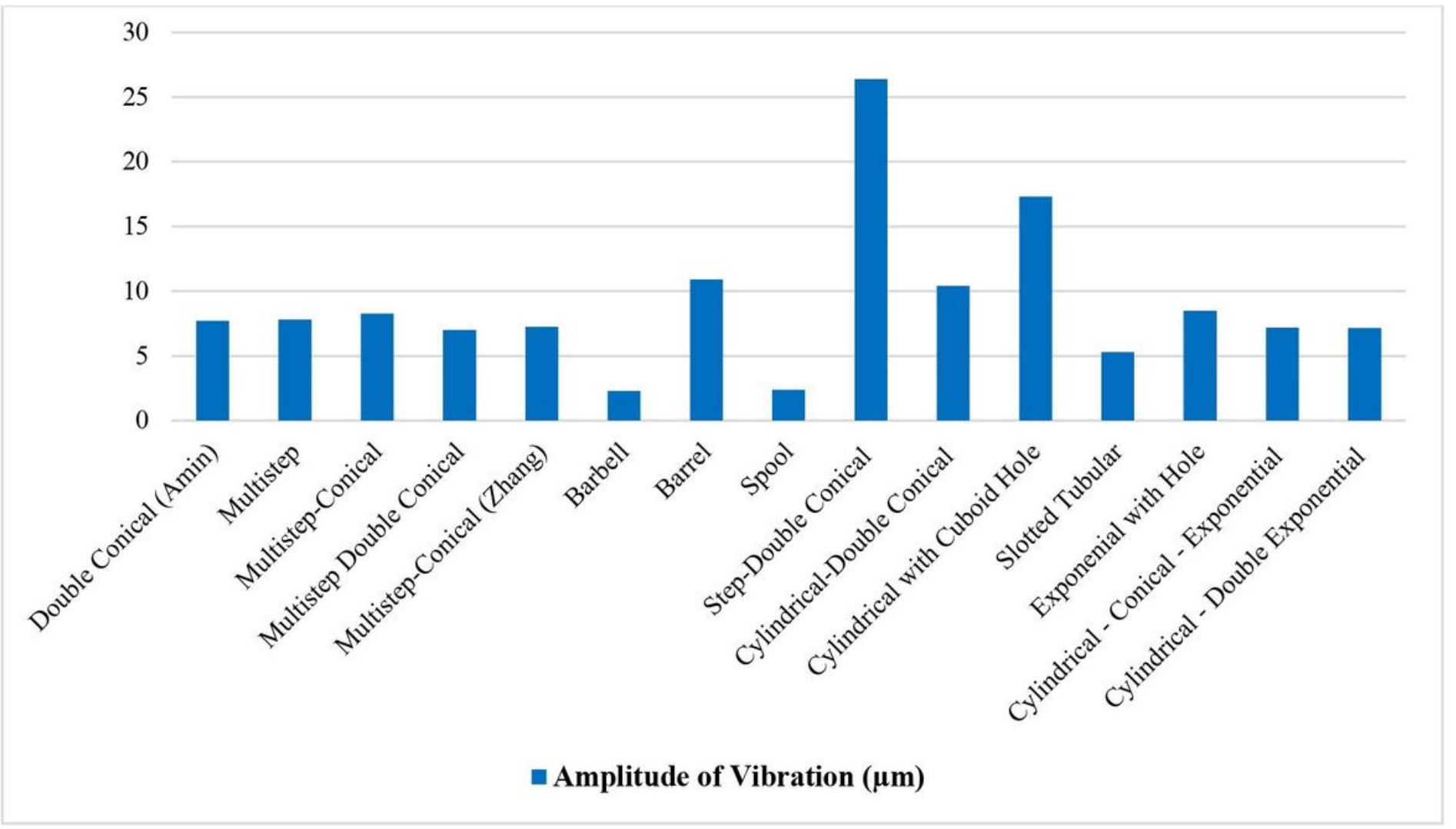

Figure 10

Comparison of amplitude of vibration for specialized hybrid ultrasonic horn designs 


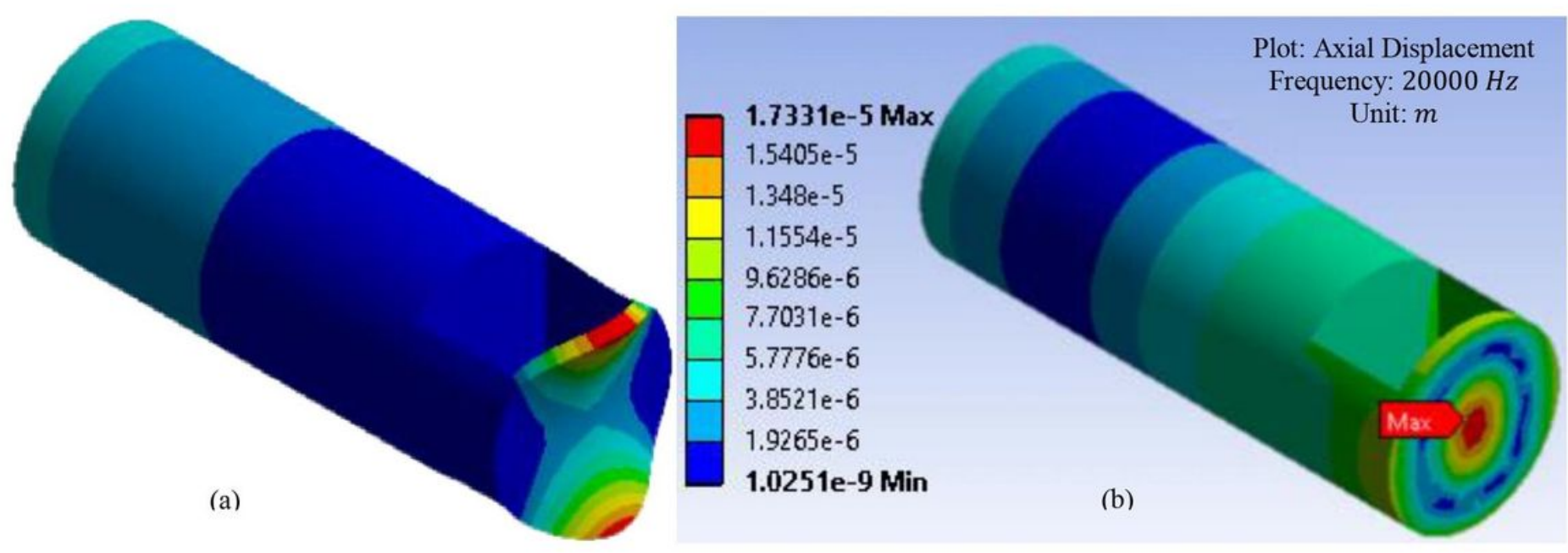

\section{Figure 11}

Countour polts for cylindrical horn with cuboid hole illustrating a) second axial mode of vibration, b) axial displacement and amplitude of vibration 


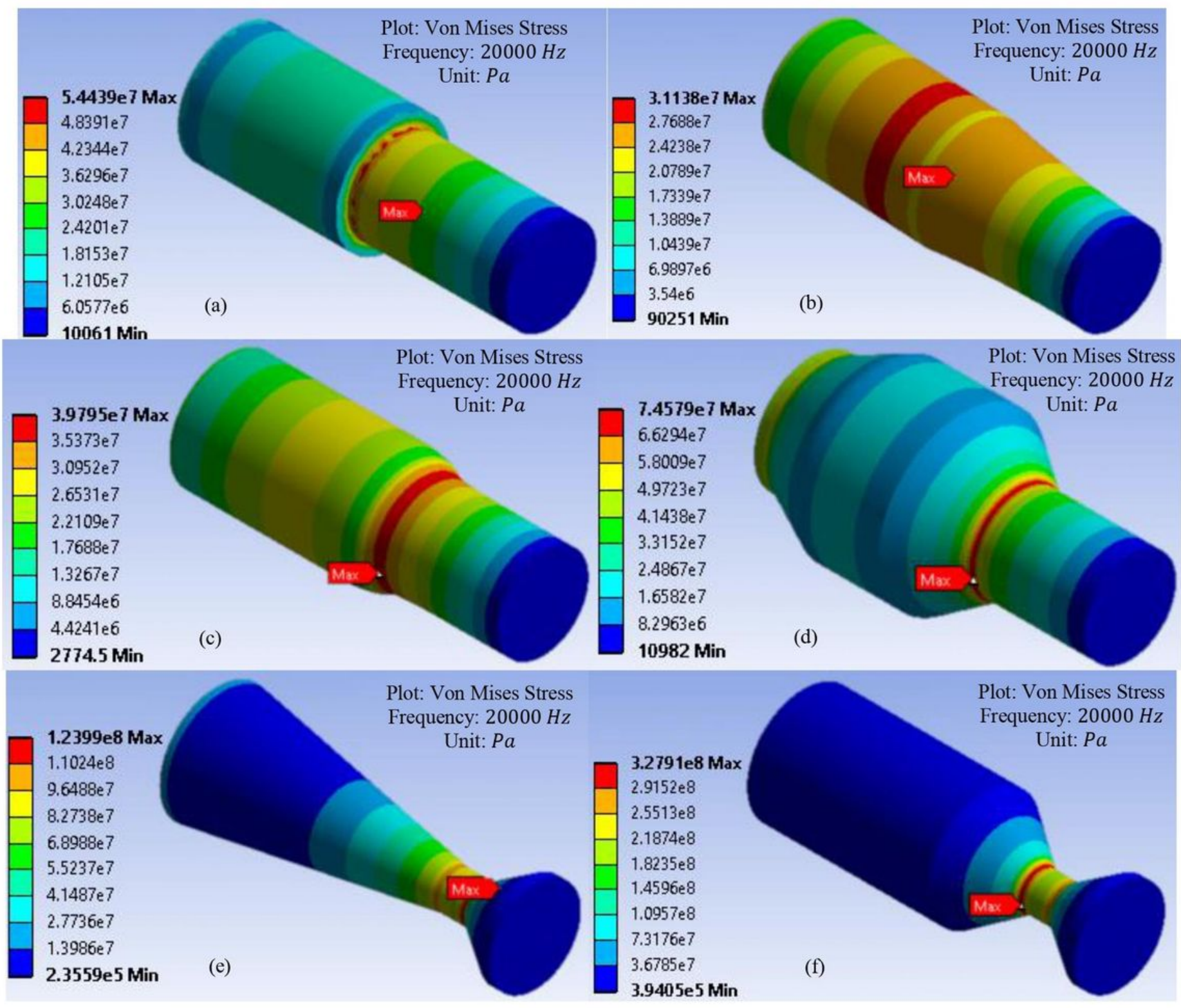

\section{Figure 12}

Contour plots of Von Mises Stress for a) step horn, b) step-catenoidal horn, c) step-Bezier horn, d) barrel horn, e) cylindrical-double conical horn, e) step-double conical horn 


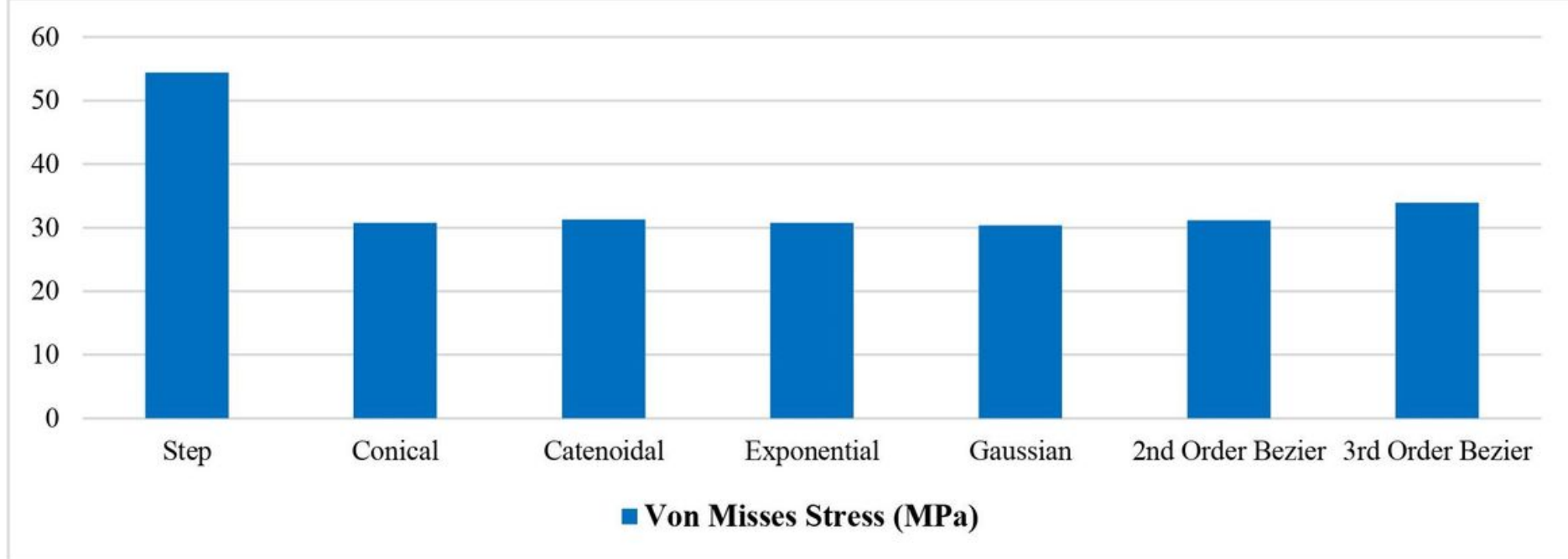

\section{Figure 13}

Comparison of Von Mises stresses for standard ultrasonic horn designs

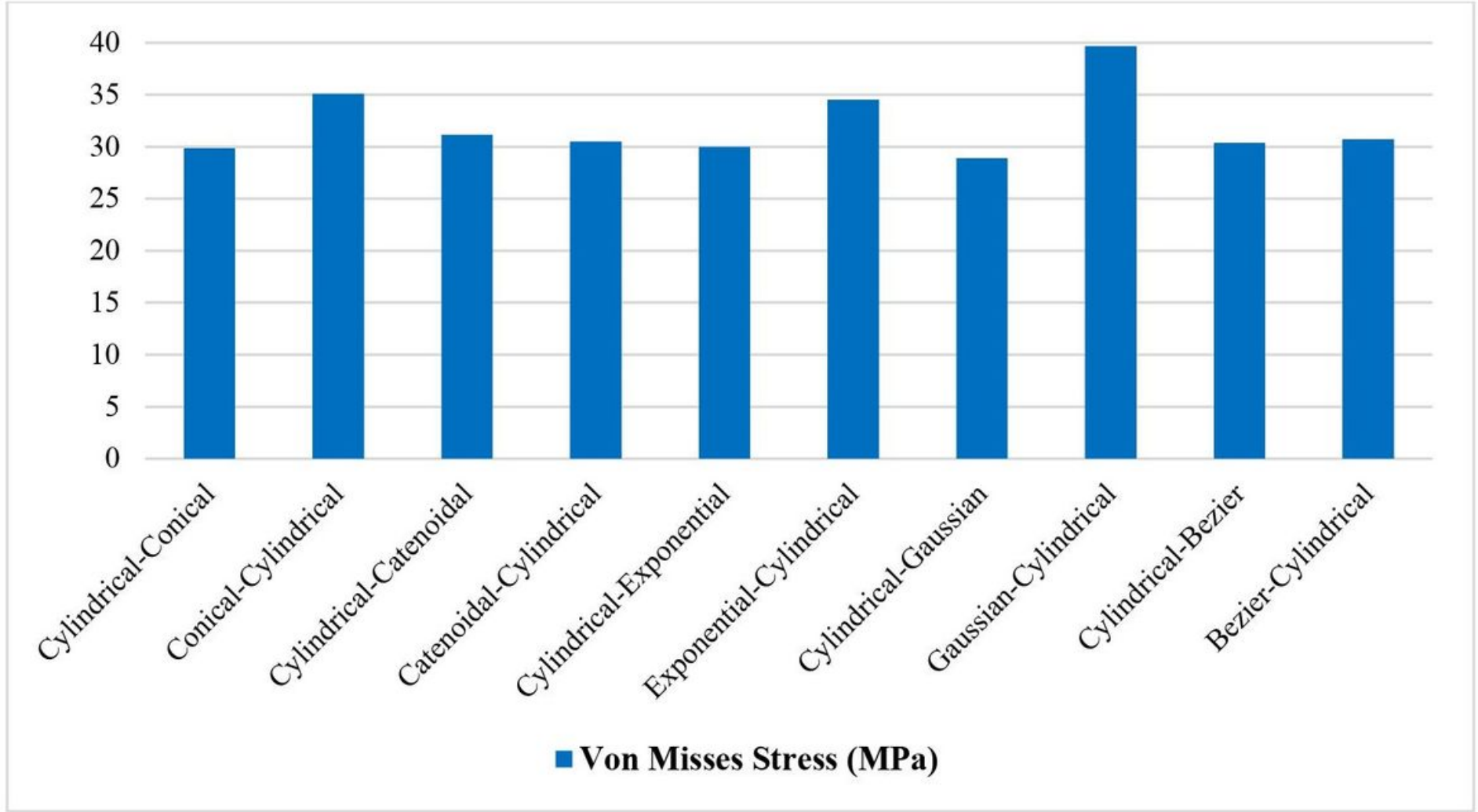

Figure 14

Comparison of Von Mises stresses for cylindrical hybrid ultrasonic horn designs 


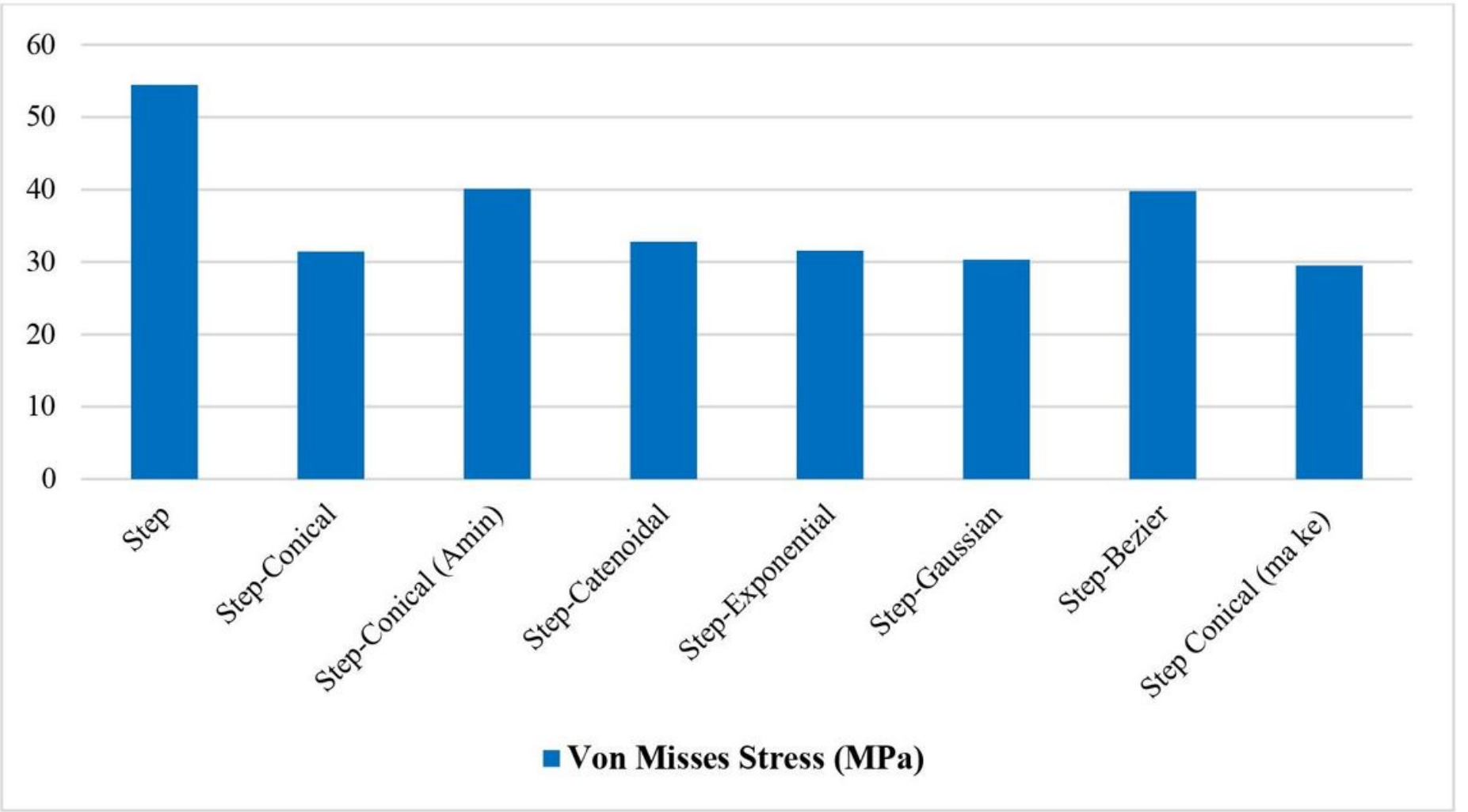

\section{Figure 15}

Comparison of Von Mises stresses for step hybrid ultrasonic horn designs

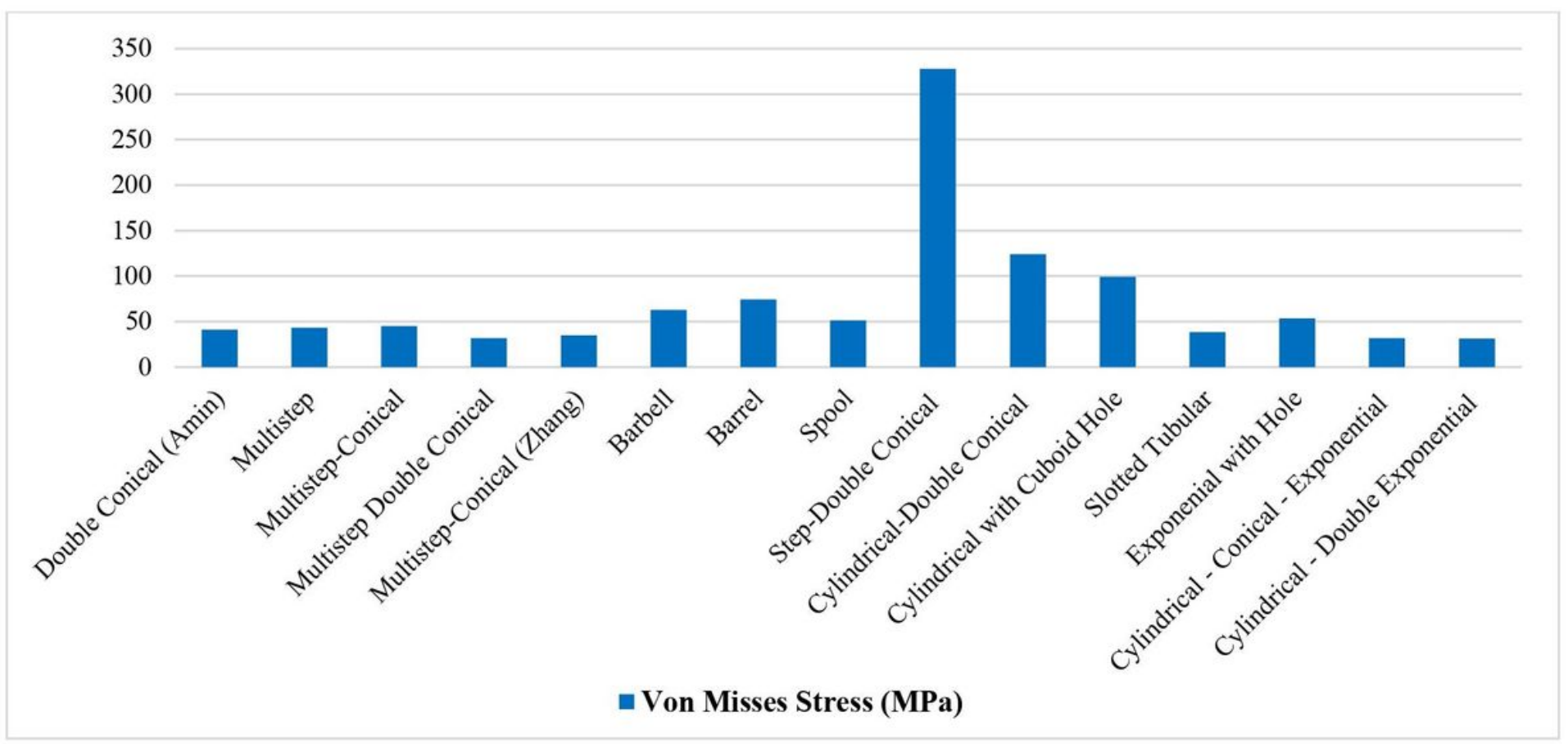

Figure 16

Comparison of Von Mises stresses for specialized complex hybrid ultrasonic horn designs 


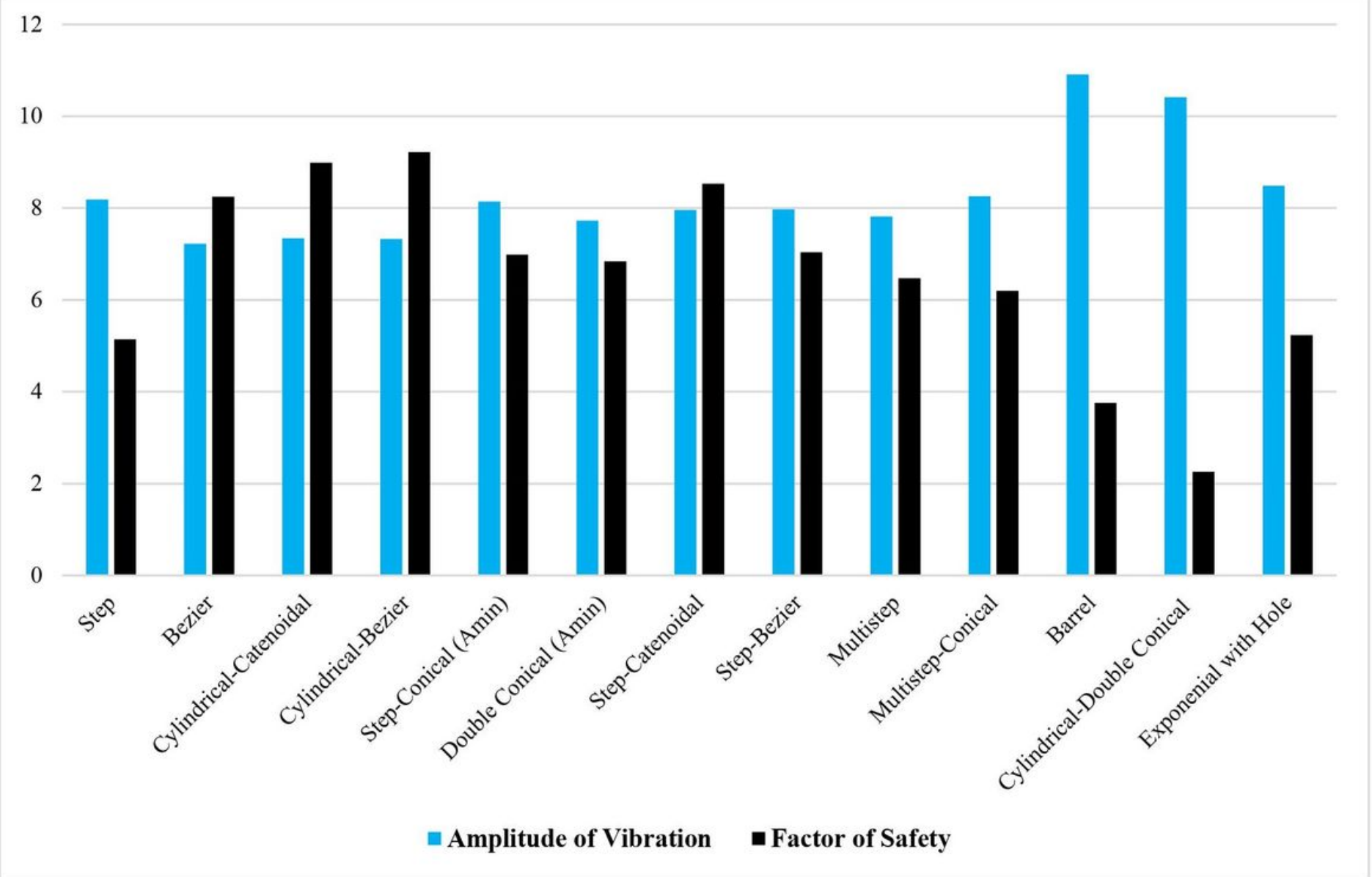

Figure 17

Comparison of vibration amplitude and factor of safety for better ultrasonic horn designs

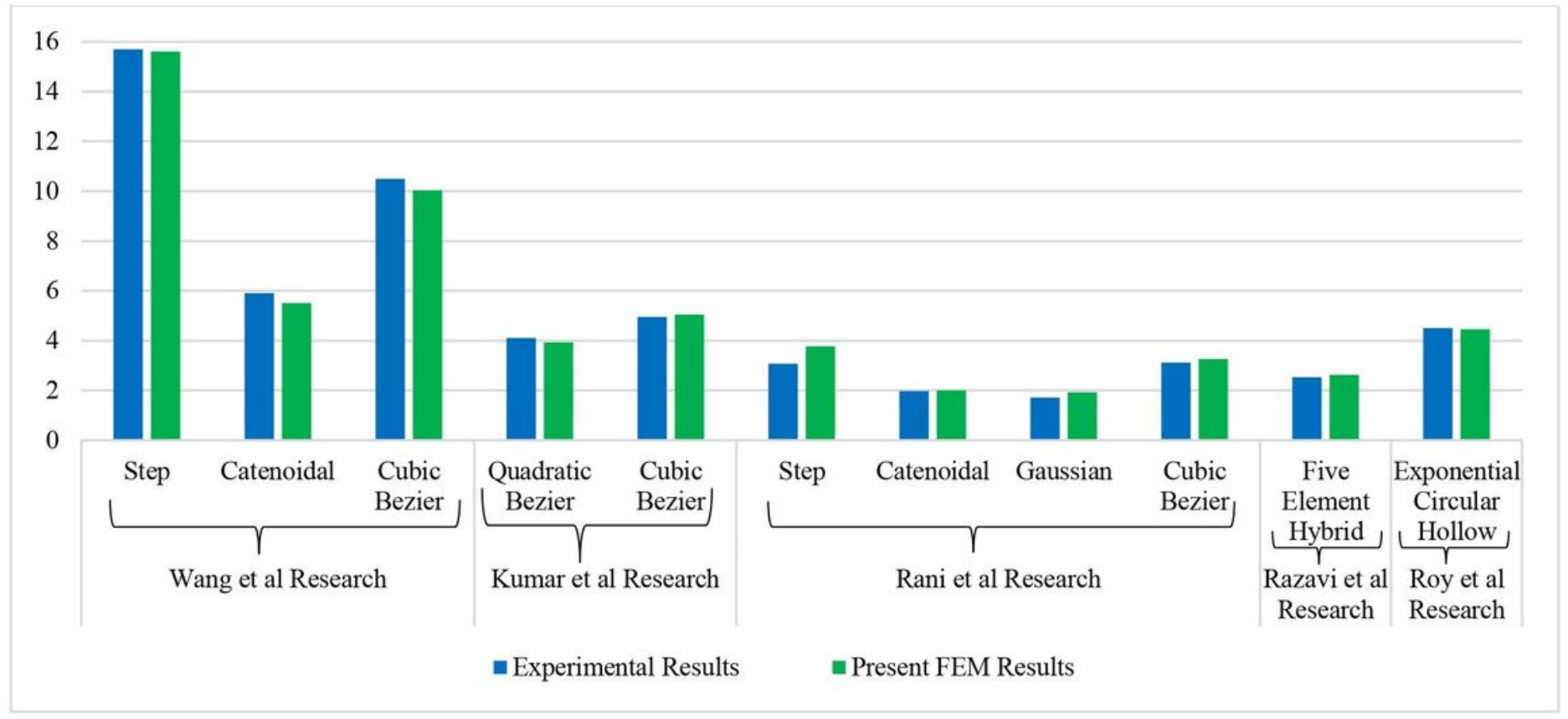


Validation of Magnification factor 